\title{
Recommandations sur la conception, le calcul, l'exécution et le contrôle des fondations d'éoliennes
}

\section{Membres du groupe de travail (( Fondations d'éoliennes ))}

Président

Berthelot Patrick Bureau Veritas

Secrétaires

Glandy Michel

Soletanche-Bachy-Pieux

Lamadon Thierry

Bureau Veritas

Membres rédacteurs

Aguado Pascal

Apave

Carpinteiro Luis

Socotec

Dano Christophe

École Centrale Nantes

Durand Daniel

Bureau Veritas

Durand Frédéric

Fugro

Gauthey J-Robert

Spie Fondations

Jandel Éric

Fondasol

Lambert Serge

Keller

Martin Alexander

CTE

Plomteux Cyril

Ménard

Thorel Luc

LCPC

Ont également participé à la rédaction

Antoinet Éric

Antéa

Bersch Matias

CTE

Bourne Gilles

Alios

Bretelle Sylvie

De Muynck Pascale

Cathie-Associates

Denois Thierry

EDF-EN

Le Kouby Alain

EDF-EN

Liausu Philippe

LCPC

Mazaré Bruno

Ménard

Pal Olivier

Egis

Reboul Michaël

Eiffage

Terrasol

Ont été consultés

Marburger

Nordex

Niedermowwe Nills

Puech Alain

Enercon

Remillon Vincent

SOLCYP

Schacknies Meik

Repower

Enercon 


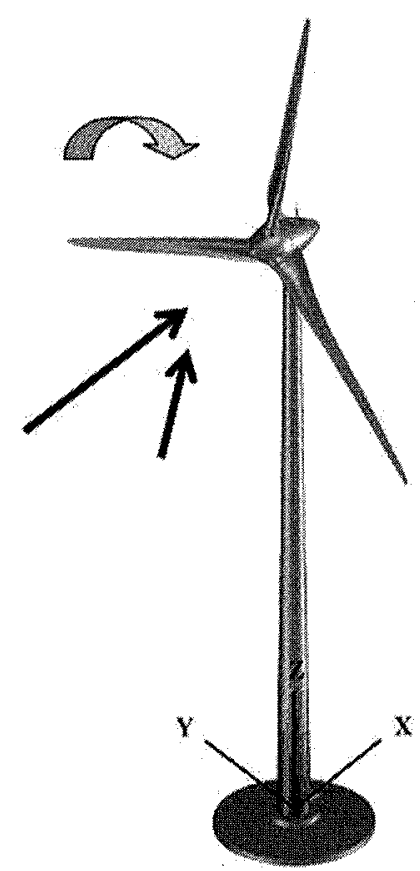

\section{Notations et unités}

\section{Notations latines}

\begin{tabular}{|c|c|c|}
\hline$A_{p}$ & Section droite de l'inclusion ou de la colonne & {$\left[\mathrm{m}^{2}\right]$} \\
\hline$A_{\text {sw }}$ & Aire de la section des armatures d'effort tranchant & {$\left[\mathrm{m}^{2}\right]$} \\
\hline B & Largeur de la fondation "comprimant le sol ») & {$[\mathrm{m}]$} \\
\hline $\mathrm{c}$ & $=2 / \mathrm{n} / \varnothing_{2} / \mathrm{K}_{\mathrm{pv}} / \varnothing_{2} \mathrm{~K}_{\mathrm{ph}} \varnothing_{1} \mathrm{lo}^{3}$ & \\
\hline $\mathrm{C}^{\prime}$ & Cohésion effective & {$[\mathrm{Pa}]$} \\
\hline $\mathrm{C}_{\max }$ & Coefficient tenant compte de la consistance qu'il est nécessaire de donner au béton & \\
\hline $\mathrm{d}$ & $\mathrm{d}=1-\mathrm{nc} /(1+\mathrm{nc})$ & \\
\hline$d_{x}$ & Diamètre des grains solides à $\mathrm{x} \%$ de passant & {$[\mathrm{m}]$} \\
\hline $\mathrm{d}_{1}$ & Longueur du mécanisme de rupture & {$[\mathrm{m}]$} \\
\hline $\mathrm{d}_{2}$ & Longueur du mécanisme de rupture & {$[\mathrm{m}]$} \\
\hline e & Excentrement de la charge verticale $=\mathrm{M} / \mathrm{V}$ & [m] \\
\hline $\mathrm{E}$ & Module d'Young (pour des déformations comprises entre $10^{-3}$ et $10^{-4}$ ) & [Pa] \\
\hline $\mathrm{E}_{\mathrm{c}}$ & Module sphérique (Ménard) & {$[\mathrm{Pa}]$} \\
\hline$E_{d}$ & Module déviatorique (Ménard) & {$[\mathrm{Pa}]$} \\
\hline $\mathrm{E}_{\text {eq }}$ & Module de déformation équivalent & [Pa] \\
\hline$E_{\max }$ & Module d'Young pour une déformation de l'ordre de $10^{-6}$ & {$[\mathrm{~Pa}]$} \\
\hline $\mathrm{E}_{\text {oedl }}$ & Module œdométrique & {$[\mathrm{Pa}]$} \\
\hline$E_{\text {yst }}$ & Module d'Young pour une déformation de l'ordre de $10^{-2}$ & [Pa] \\
\hline$E_{M}$ & Module conventionnel déterminé lors de l'essai pressiométrique Ménard normalisé & {$[\mathrm{Pa}]$} \\
\hline $\mathrm{E}_{\mathrm{mEq}}$ & Moyenne harmonique des $\mathrm{E}_{\mathrm{M}}$ & {$[\mathrm{Pa}]$} \\
\hline EV1 & Essai de plaque : module de premier chargement & {$[\mathrm{Pa}]$} \\
\hline EV2 & Essai de plaque : module de deuxième chargement & {$[\mathrm{Pa}]$} \\
\hline
\end{tabular}




\begin{tabular}{|c|c|c|}
\hline $\mathrm{E}_{y}$ & Module d’Young du matériau de l'élément rigide & [Pa] \\
\hline$f_{c}^{*}$ & Valeur caractéristique de la résistance conventionnelle du béton ou coulis & [Pa] \\
\hline$f_{\mathrm{cd}}$ & Contrainte de calcul en compression de l'inclusion & {$[\mathrm{Pa}]$} \\
\hline$f_{c j}$ & Résistance à la compression du matériau de l'élément rigide & [Pa] \\
\hline$f_{c k}$ & Résistance caractéristique en compression du béton mesurée sur cylindres à 28 jours & {$[\mathrm{Pa}]$} \\
\hline$f_{c k}(t)$ & Résistance caractéristique en compression du béton mesurée sur cylindres au temps t & {$[\mathrm{Pa}]$} \\
\hline$f_{c k}^{*}$ & Résistance caractéristique à la compression du béton du coulis ou mortier d'une inclusion & {$[\mathrm{Pa}]$} \\
\hline$f_{c t}$ & Résistance en traction directe du béton & {$[\mathrm{Pa}]$} \\
\hline$f_{c t d}$ & Résistance de calcul en traction du béton & {$[\mathrm{Pa}]$} \\
\hline$f_{\text {ctk0.05 }}$ & Fractile à $5 \%$ de la résistance caractéristique en traction du béton & {$[\mathrm{Pa}]$} \\
\hline$f_{\text {ctm }}$ & Valeur moyenne de la résistance en traction directe du béton & {$[\mathrm{Pa}]$} \\
\hline$f_{\text {cyd }}$ & Résistance de calcul en cisaillement et compression du béton & {$[\mathrm{Pa}]$} \\
\hline$f_{\text {rwd }}$ & Résistance de calcul de l'acier $\left(=\mathrm{f}_{\mathrm{e}} / \gamma_{\mathrm{s}}\right)$ à l'ELU & \\
\hline$f_{s}$ & Frottement latéral unitaire local (au pénétromètre statique) & [Pa] \\
\hline$f_{e}$ & Limite élastique du matériau dans le cas d'inclusions métalliques & {$[\mathrm{Pa}]$} \\
\hline$F_{\text {eau }}$ & Force verticale de soulèvement exercé par l'eau sur le massif & [N] \\
\hline $\mathrm{F}_{z}$ ou V & Force verticale de compression exercée sur le massif & {$[N]$} \\
\hline $\mathrm{F}_{\mathrm{zELU}}$ & Compression & {$[\mathrm{N}]$} \\
\hline $\mathrm{F}_{\mathrm{zmin}}$ & Charge verticale minimale de compression transmise au sol par la semelle & {$[\mathrm{N}]$} \\
\hline you $\gamma_{\circ}$ & Accélération de la pesanteur terrestre & {$\left[\mathrm{m} / \mathrm{s}^{2}\right]$} \\
\hline G & Module de cisaillement (pour des déformations comprises entre $10^{-3}$ et $10^{-4}$ ) & {$[\mathrm{Pa}]$} \\
\hline$G_{\text {coldyn }}$ & Module de cisaillement à $10^{-4}$ dans les colonnes ballastées & {$[\mathrm{Pa}]$} \\
\hline $\mathrm{G}_{\mathrm{eq}}$ & Module de cisaillement équivalent du système sol-colonne pour les plages de déformations de $10^{-3}$ à $10^{-4}$ & {$[\mathrm{~Pa}]$} \\
\hline$G_{\max }$ & Module de cisaillement à $10^{-6}$ de distorsion & {$[\mathrm{Pa}]$} \\
\hline$G_{\text {dyneq }}$ & Module de cisaillement dynamique équivalent & {$[\mathrm{Pa}]$} \\
\hline $\mathrm{G}_{\text {soldyn }}$ & Module de cisaillement à $10^{-4}$ dans le sol autour des colonnes ballastées & {$[\mathrm{Pa}]$} \\
\hline h & Profondeur d'encastrement du massif & [m] \\
\hline $\mathrm{h}_{1}$ & Enfoncement mini de la semelle & [m] \\
\hline $\mathrm{h}_{1}$ & Longueur du mécanisme de rupture & {$[\mathrm{m}]$} \\
\hline $\mathrm{h}_{2}$ & Enfoncement maxi de la semelle & [m] \\
\hline $\mathrm{h}_{2}$ & Longueur du mécanisme de rupture & [m] \\
\hline $\mathrm{h}_{\mathrm{i}}$ & Effort tranchant en tête de colonne fictive située dans le matelas au droit de l'inclusion & {$[\mathrm{N}]$} \\
\hline$h_{r}$ & Épaisseur de matelas minimale & [m] \\
\hline $\mathrm{h}_{\mathrm{s}}$ & Effort de cisaillement repris en sous face de semelle par le matelas & [m] \\
\hline $\mathrm{H}$ & Force horizontale exercée sur le massif & {$[N]$} \\
\hline $\mathrm{H}_{\mathrm{ELU}}$ & Force horizontale exercée sur le massif à l'ELU & {$[N]$} \\
\hline $\mathrm{H}_{\text {mat }}$ & Épaisseur du matelas & [m] \\
\hline$I_{\delta^{\prime}}, i_{\beta}, i_{e}$ & Facteurs de correction pour une fondation superficielle & \\
\hline$i_{\delta \beta}$ & Facteur de réduction de portance pour la combinaison d'une charge inclinée et d'une pente & \\
\hline I & Inertie de la semelle & {$\left[\mathrm{m}^{4}\right]$} \\
\hline I & Inertie d'un pieu & {$\left[\mathrm{m}^{4}\right]$} \\
\hline I & Inertie de l'inclusion rigide & {$\left[\mathrm{m}^{4}\right]$} \\
\hline
\end{tabular}




\begin{tabular}{|c|c|c|}
\hline $\mathrm{J}$ & $n \varnothing_{2}{ }^{2} / 8$ & {$\left[\mathrm{~m}^{2}\right]$} \\
\hline $\mathrm{k}_{1}$ & Coefficient fonction de la méthode de forage & {$[-]$} \\
\hline $\mathrm{k}_{2}$ & Coefficient fonction de l'élancement & {$[-]$} \\
\hline $\mathrm{k}_{3}$ & Coefficient fonction du type d'ouvrage & {$[-]$} \\
\hline $\mathrm{k}_{\mathrm{c}}$ & Coefficient de portance & {$[-]$} \\
\hline $\mathrm{k}_{\mathrm{p}}$ & Coefficient de portance & {$[-]$} \\
\hline $\mathrm{k}_{\mathrm{y}}$ & Raideur verticale & {$\left[\mathrm{N} / \mathrm{m}^{2} / \mathrm{m}\right]$} \\
\hline $\mathrm{K}_{\mathrm{ph}}$ & Raideur horizontale de l'inclusion ou du pieu en tête & {$[\mathrm{N} / \mathrm{m}]$} \\
\hline $\mathrm{K}_{\mathrm{pv}}$ & Raideur verticale de l'inclusion ou du pieu & {$[\mathrm{N} / \mathrm{m}]$} \\
\hline $\mathrm{K}_{\mathrm{x}} \mathrm{K}_{\mathrm{y}} \mathrm{K}_{\mathrm{z}}$ & Raideur horizontale minimale imposée du massif selon les axes xx, yy et zz & {$[\mathrm{N} / \mathrm{m}]$} \\
\hline $\mathrm{K}_{\mathrm{h}}$ & Coefficient de réaction de la fondation & {$[\mathrm{N} / \mathrm{m}]$} \\
\hline $\mathrm{K}_{\mathrm{s}}$ & Raideur du sol & {$[\mathrm{N} / \mathrm{m}]$} \\
\hline $\mathrm{K}_{\mathrm{v}}$ & Raideur verticale du sol & {$[\mathrm{N} / \mathrm{m}]$} \\
\hline $\mathrm{K}_{\mathrm{vs}}$ & Raideur verticale statique $\mathrm{K}_{\mathrm{vs}}=\mathrm{q} / \mathrm{w}$ & {$[\mathrm{N} / \mathrm{m}]$} \\
\hline $\mathrm{K}_{\varphi}$ & Raideur en rotation & {$[\mathrm{Nm} / \mathrm{rad}]$} \\
\hline $\mathrm{K}_{\varphi \mathrm{CCT}}$ & Raideur en rotation à CT (Court Terme) & {$[\mathrm{Nm} / \mathrm{rad}]$} \\
\hline $\mathrm{K}_{\text {QLT }}$ & Raideur en rotation à LT (Long Terme) & {$[\mathrm{Nm} / \mathrm{rad}]$} \\
\hline $\mathrm{K}_{\varphi \mathrm{NS}}$ & Raideur en rotation quand le massif n'est pas soulevé & {$[\mathrm{Nm} / \mathrm{rad}]$} \\
\hline$K_{\varphi p, d y n}$ & Raideur en rotation à petite déformation (de $10^{-5}$ à $10^{-3}$ ) & [Nm/rad] \\
\hline $\mathrm{L}$ & Longueur d'une fondation (inclusion, pieu ou colonne ballastée) & {$[\mathrm{m}]$} \\
\hline$l_{0}$ & Longueur de transfert & {$[\mathrm{m}]$} \\
\hline M & Moment de renversement appliqué sur le massif & [Nm] \\
\hline$M_{i}$ & Moment maxi en tête de pieu & [Nm] \\
\hline $\mathrm{M}^{\prime}$ & $=M_{x y}-n \cdot M_{i}$ & {$[\mathrm{Nm}]$} \\
\hline $\mathrm{M}_{\mathrm{ELU}}$ & Moment à l’ELU & {$[\mathrm{Nm}]$} \\
\hline M & Moment de renversement & {$[\mathrm{Nm}]$} \\
\hline $\mathrm{m}^{\prime}$ & $=(n-1) / n$ & {$[-]$} \\
\hline$n$ & Porosité & {$[-]$} \\
\hline $\mathrm{n}$ & Facteur d'amélioration $=\sigma_{\text {app }} / \sigma_{\text {sol }}$ & \\
\hline $\mathrm{n}$ & Nombre de colonnes sous la surface de référence $S_{\text {ref }}$ & {$[-]$} \\
\hline $\mathrm{n}$ & Nombre d'inclusions ou de pieux & {$[-]$} \\
\hline $\mathrm{Nc}$ & Terme de cohésion & {$[-]$} \\
\hline $\mathrm{Nq}$ & Terme de profondeur & {$[-]$} \\
\hline$p_{f}$ & Pression de fluage & {$[\mathrm{Pa}]$} \\
\hline$p_{1}$ & Pression limite pressiométrique & {$[\mathrm{Pa}]$} \\
\hline$p_{1^{*}}$ & Pression limite pressiométrique nette & {$[\mathrm{Pa}]$} \\
\hline $\mathrm{p}_{\text {lci }}$ & Pression limite de calcul $p_{\mathrm{lci}}=\mathrm{p}_{\mathrm{li}}{ }^{*}\left[\left(1+\alpha_{\mathrm{i}}\right)^{2}\right]$ & {$[\mathrm{Pa}]$} \\
\hline $\mathrm{p}_{\mathrm{le}}$ & Pression limite équivalente & {$[\mathrm{Pa}]$} \\
\hline $\mathrm{p}_{\mathrm{le}}{ }^{*}$ & Pression limite nette équivalente & {$[\mathrm{Pa}]$} \\
\hline $\mathrm{p}_{\mathrm{li}}$ & Pression limite mesurée de la tranche « $\mathrm{i})$ & {$[\mathrm{Pa}]$} \\
\hline$p_{\operatorname{lmax}}$ & Pression limite maximale mesurée & {$[\mathrm{Pa}]$} \\
\hline $\mathrm{p}_{\text {lmin }}$ & Pression limite minimale mesurée & {$[\mathrm{Pa}]$} \\
\hline
\end{tabular}




\begin{tabular}{|c|c|c|}
\hline q & $\mathrm{K}_{\mathrm{vs}}=\mathrm{q} / \mathrm{w}$ & [Pa] \\
\hline $\mathrm{q}_{0}^{\prime}$ & $=\rho^{\prime} \times \gamma \times z$ & [Pa] \\
\hline$q_{1}$ & Contrainte en sous-face du matelas (au droit de l'inclusion) & [Pa] \\
\hline $\mathrm{q}_{2}$ & Contrainte en sous-face du matelas (au droit du sol) & {$[\mathrm{Pa}]$} \\
\hline $\mathrm{q}_{\mathrm{a}}$ & Contrainte dans les colonnes ballastées & {$[\mathrm{Pa}]$} \\
\hline $\mathrm{q}_{\text {app }}^{\prime}$ & Contrainte reprise moyenne par le sol sur la maille & [Pa] \\
\hline$q_{c}$ & Résistance de pointe (ou résistance de cône) & {$[\mathrm{Pa}]$} \\
\hline qce & Résistance de pointe (ou résistance de cône) équivalente & [Pa] \\
\hline qcci & Résistance de pointe de calcul de la couche i & [Pa] \\
\hline $\mathrm{q}_{\mathrm{ci}}$ & Résistance de pointe de la couche i & {$[\mathrm{Pa}]$} \\
\hline $\mathrm{q}_{\mathrm{cEq}}$ & Moyenne harmonique de qc & {$[\mathrm{Pa}]$} \\
\hline $\mathrm{q}_{\mathrm{cm}}$ & Résistance de pointe moyenne & {$[\mathrm{Pa}]$} \\
\hline $\mathrm{q}_{\mathrm{col}}$ & Contrainte dans les colonnes & {$[\mathrm{Pa}]$} \\
\hline$q_{d}$ & Résistance de pointe au pénétromètre dynamique & {$[\mathrm{Pa}]$} \\
\hline $\mathrm{q}_{\text {mat }}$ & Contrainte admissible dans le matelas en tête d'inclusion & [Pa] \\
\hline $\mathrm{q}_{\mathrm{p}}$ & Portance du sol sous la semelle & {$[\mathrm{Pa}]$} \\
\hline $\mathrm{q}_{\mathrm{p}}$ & Contrainte transmise à l'inclusion par le matelas & [Pa] \\
\hline$q_{p: 1}$ & Résistance unitaire de pointe des inclusions & {$[\mathrm{Pa}]$} \\
\hline $\mathrm{q}_{\mathrm{r}}$ & Contrainte verticale de rupture $q_{r} d^{\prime} u n e$ colonne isolée & {$[\mathrm{Pa}]$} \\
\hline $\mathrm{q}_{\mathrm{re}}$ et $\mathrm{q}_{\mathrm{rp}}$ & Cf. définitions $§ 5.4$ dans les « Recommandations colonnes ballastées du CFMS (RFG n 136, 2011) ) & [Pa] \\
\hline $\mathrm{q}_{\text {ref }}$ & Contrainte maxi appliquée sur le sol & {$[\mathrm{Pa}]$} \\
\hline $\mathrm{q}_{\text {refELS }}$ & Contrainte de calcul ELS & {$[\mathrm{Pa}]$} \\
\hline $\mathrm{q}_{\text {refELU }}$ & Contrainte de calcul ELU & {$[\mathrm{Pa}]$} \\
\hline $\mathrm{q}_{\mathrm{S}}$ & Frottement latéral unitaire limite & {$[\mathrm{Pa}]$} \\
\hline $\mathrm{q}_{\mathrm{S}}$ & Contrainte sous la semelle & {$[\mathrm{Pa}]$} \\
\hline $\mathrm{q}_{\mathrm{S}}$ & Contrainte transmise au sol compressible par le matelas & {$[\mathrm{Pa}]$} \\
\hline $\mathrm{q}_{\mathrm{s} ; \mathrm{l}}$ & Contrainte de rupture sous la semelle & {$[\mathrm{Pa}]$} \\
\hline $\mathrm{q}_{\mathrm{sol}}$ & Portance globale (cas des colonnes ballastées) & [Pa] \\
\hline $\mathrm{Q}_{\mathrm{col}}$ & Valeur de la sollicitation maximale dans la colonne ballastée & {$[\mathrm{N}]$} \\
\hline$Q_{\mathrm{i}}$ & Valeur de sollicitation de la colonne fictive située dans le matelas au droit d'une inclusion & {$[N]$} \\
\hline$Q_{\max }$ & $\begin{array}{l}\text { Charge verticale maximum de compression par élément rigide vertical induite par le moment } \\
\text { de renversement }\end{array}$ & {$[N]$} \\
\hline$Q_{p}$ & Charge verticale par inclusion sous chargement centré & {$[N]$} \\
\hline$Q_{\text {sem }}$ & Charge en compression repris par le sol en sous face de semelle & {$[\mathrm{N}]$} \\
\hline r & Rayon du massif circulaire de même section que le massị d'éolienne & {$[\mathrm{m}]$} \\
\hline $\mathrm{r}^{*}$ & Rayon du massif circulaire de même section que la surface entièrement comprimée & \\
\hline $\mathrm{R}_{\mathrm{b}}$ & Portance en pointe de l'inclusion & {$[\mathrm{N}]$} \\
\hline $\mathrm{R}_{\mathrm{f}}$ & Rapport de frottement & {$[-]$} \\
\hline $\mathrm{R}_{\mathrm{s}}$ & Portance en frottement de l'inclusion & {$[N]$} \\
\hline$s_{t}$ & Espacement des spires & {$[\mathrm{m}]$} \\
\hline s & Tassement & {$[\mathrm{m}]$} \\
\hline $\mathrm{S}$ & Section pleine d'un pieu & {$\left[\mathrm{m}^{2}\right]$} \\
\hline $\mathrm{S}_{\text {col }}$ & Section comprimée de la colonne & {$\left[\mathrm{m}^{2}\right]$} \\
\hline
\end{tabular}




\begin{tabular}{|c|c|c|}
\hline $\mathrm{S}_{\text {comp }}$ & Surface réellement comprimée sous la semelle & {$\left[\mathrm{m}^{2}\right]$} \\
\hline $\mathrm{S}_{\mathrm{c}}$ & Section droite de la dallette & {$\left[\mathrm{m}^{2}\right]$} \\
\hline $\mathrm{S}_{\mathrm{sem}}$ & Surface totale de la semelle & {$\left[\mathrm{m}^{2}\right]$} \\
\hline $\mathrm{S}_{\text {maille }}$ & Surface d'une maille & {$\left[\mathrm{m}^{2}\right]$} \\
\hline $\mathrm{S}_{\mathrm{r}}$ & Degré de saturation & {$[-]$} \\
\hline $\mathrm{S}_{\text {ref }}$ & Surface comprimée de la lunule & {$\left[\mathrm{m}^{2}\right]$} \\
\hline $\mathrm{T}(\mathrm{z})$ & Frottement mobilisable & {$[N]$} \\
\hline $\mathrm{v}$ & Cf. J/v et vaut $\Phi_{2} / 2$ & {$[\mathrm{~m}]$} \\
\hline $\mathrm{V}_{\mathrm{i}}$ & Effort tranchant maximum dans une inclusion rigide & {$[\mathrm{N}]$} \\
\hline $\mathrm{V}_{\mathrm{P}}$ & Vitesse des ondes de compression (dites primaires) & {$[\mathrm{m} / \mathrm{s}]$} \\
\hline $\mathrm{V}_{\mathrm{Rd}, \mathrm{s}}$ & Effort tranchant admissible à l'ELU du pieu ou de l'IR en fonction de l'acier mis en place & {$[\mathrm{N}]$} \\
\hline$V_{\text {rdmax }}$ & Effort tranchant admissible à l'ELU du pieu ou de l'IR en fonction de la résistance du béton & {$[\mathrm{N}]$} \\
\hline $\mathrm{V}_{\mathrm{S}}$ & Vitesse des ondes de cisaillement (dites secondaires) & {$[\mathrm{m} / \mathrm{s}]$} \\
\hline$w_{c}$ & Tassement sphérique & {$[\mathrm{m}]$} \\
\hline$w_{\mathrm{d}}$ & Tassement déviatorique & {$[\mathrm{m}]$} \\
\hline w & Tassement total sous charge centrée & {$[\mathrm{m}]$} \\
\hline w & Teneur en eau & {$[-]$} \\
\hline$w r(z)$ & Tassement relatif & {$[\mathrm{m}]$} \\
\hline $\mathrm{w}_{\mathrm{s}}(0)$ & Enfoncement de la semelle & {$[\mathrm{m}]$} \\
\hline $\mathrm{y}^{\prime}$ & Rotation de l'inclusion ou de la semelle & rd \\
\hline$y_{\max }$ & Enfoncement maximal de la semelle & {$[\mathrm{m}]$} \\
\hline (z) & Profondeur, variable des fonctions w(z), t(z) & {$[\mathrm{m}]$} \\
\hline $\mathrm{z}$ & Bras de levier & {$[\mathrm{m}]$} \\
\hline
\end{tabular}

\section{Notations grecques}

\begin{tabular}{|c|c|c|}
\hline$\alpha$ & Coefficient rhéologique (Fascicule 62, Ménard, $=\mathrm{E}_{\mathrm{M}} / \mathrm{E}$ ) & {$[-]$} \\
\hline$\alpha$ & Inclinaison des cerces & \\
\hline$\alpha_{c w}$ & Coefficient de l’EC2 & \\
\hline$\alpha_{1}$ & $=\mathrm{E}_{\mathrm{oed}} / \mathrm{q}_{\mathrm{C}^{\prime}}$ & {$[-]$} \\
\hline$\alpha_{2}$ & $=\sigma_{\mathrm{max}} / \sigma_{\mathrm{moy}}$ & {$[-]$} \\
\hline$\alpha_{i}$ & Compris entre 0 et 1,5 & \\
\hline$\alpha$ & $\begin{array}{l}\text { Taux de recouvrement (ou de couverture) d'un sol renforcé par inclusions rigides, égal au rapport } \\
\text { de l'aire couverte par les têtes d'inclusions et l'aire totale de la surface traitée }\end{array}$ & \\
\hline$\alpha_{c c}$ & Coefficient qui dépend de la présence ou non d’une armature & \\
\hline$\alpha_{c p l}$ & Coefficient de l’EC2 dépendant de la présence ou non d’armature & \\
\hline$\beta$ & $\begin{array}{l}\text { Taux d'incorporation du renforcement par colonnes ballastées, égal au rapport de l'aire couverte } \\
\text { par les têtes de colonnes et l'aire totale de la surface traitée }=A_{\text {col }} / S_{\text {maille }}\end{array}$ & {$[-]$} \\
\hline$\beta$ & Coefficient réducteur appliqué à la raideur en rotation en fonction du pourcentage de surface comprimée & [-] \\
\hline$\beta_{1}$ & $\begin{array}{l}\text { Coefficient réducteur appliqué à la raideur en rotation en fonction du pourcentage de surface } \\
\text { comprimée } \mathrm{K}_{\varphi} / \mathrm{K}_{\varphi N S}\end{array}$ & {$[-]$} \\
\hline$\delta$ & Angle de frottement entre la semelle et le sol & {$[\mathrm{rad}$} \\
\hline
\end{tabular}




\begin{tabular}{|c|c|c|}
\hline$\varepsilon$ & Déformation linéique $(\Delta \mathrm{l} / \mathrm{l}, \Delta \mathrm{l}$ déplacement dans le sens de l'élément 1$)$ & $\%$ \\
\hline$\varnothing$ & Diamètre équivalent du massif & [m] \\
\hline$\varnothing_{1}$ & Diamètre de l'inclusion, du pieu ou de la colonne ballastée & [m] \\
\hline$\varnothing_{2}$ & Diamètre du cercle où sont situées les inclusions les plus excentrées & [m] \\
\hline$\Phi$ & Angle de rotation de l'éolienne autour d'un axe horizontal & {$[\mathrm{rad}]$} \\
\hline$\varphi^{\prime}$ & Angle de frottement effectif & {$\left[{ }^{\circ}\right]$} \\
\hline$\varphi_{c}$ & Angle de frottement d'une colonne ballastée & {$\left[{ }^{\circ}\right]$} \\
\hline$\varphi_{\text {eq }}$ & Angle de frottement équivalent & {$\left[{ }^{\circ}\right]$} \\
\hline$\varphi_{\mathrm{R}}^{\prime}$ & Angle de frottement résiduel & {$\left[{ }^{\circ}\right]$} \\
\hline$\varphi_{s}$ & Angle de frottement du sol & {$\left[{ }^{\circ}\right]$} \\
\hline$\gamma$ & $\begin{array}{l}\text { Distorsion ou déformation angulaire ( } 2 \Delta \alpha \text { ou } 2 \mathrm{~d} t / \mathrm{l}, \mathrm{d} t=\text { déplacement dans le sens perpendiculaire), } \\
\text { (à ne pas confondre avec les coefficients de sécurité) }\end{array}$ & $\%$ \\
\hline$\gamma_{\mathrm{b}}$ & Coefficient de sécurité sur la pointe de l'inclusion & {$[-]$} \\
\hline$\gamma_{\mathrm{c}}$ & Coefficient partiel sur le matériau de l'inclusion & \\
\hline$\gamma_{\text {matelas }}$ ou $\gamma_{\text {mat }}$ & Coefficient de sécurité sur la rupture du matelas au poinçonnement & {$[-]$} \\
\hline$\gamma_{\mathrm{s}}$ & Coefficient de sécurité sur le frottement de l'inclusion & {$[-]$} \\
\hline$\gamma_{\mathrm{sf}}$ & Coefficient de sécurité sur le frottement semelle/sol & {$[-]$} \\
\hline$\gamma_{\text {sol }}$ & Coefficient de sécurité sur la portance du sol sous la semelle & {$[-]$} \\
\hline$\gamma_{\varphi}$ & Coefficient de sécurité sur l’angle de frottement & \\
\hline$v$ & Coefficient de Poisson & {$[-]$} \\
\hline$v_{\mathrm{Eq}}$ & Coefficient de Poisson équivalent du sol renforcé par colonnes ballastées ou par inclusions rigides & {$[-]$} \\
\hline$\sigma_{\text {clim }}$ & Résistance limite de calcul en compression & {$[\mathrm{Pa}]$} \\
\hline$\sigma_{\mathrm{col}}$ & Contrainte verticale en compression dans la colonne ballastée & {$[\mathrm{Pa}]$} \\
\hline$\sigma_{c p}$ & Contrainte moyenne de compression dans l'inclusion & {$[\mathrm{Pa}]$} \\
\hline$\sigma_{i}$ & Contrainte de compression dans la colonne fictive surmontant l'inclusion ou la colonne & {$[\mathrm{Pa}]$} \\
\hline$\sigma_{\text {mat }}^{\prime}$ & Résistance au poinçonnement & [Pa] \\
\hline$\sigma_{\max }$ & Contrainte maximale du sol sous la semelle & {$[\mathrm{Pa}]$} \\
\hline$\sigma_{\min }$ & Contrainte minimale du sol sous la semelle & {$[\mathrm{Pa}]$} \\
\hline$\sigma_{\text {moy }}$ & $\mathrm{Fz} / \mathrm{S}_{\text {sem }}$ & {$[\mathrm{Pa}]$} \\
\hline$\sigma_{s}$ & Contrainte verticale en compression hors emprise de l'inclusion ou de la colonne & {$[\mathrm{Pa}]$} \\
\hline$\theta$ & Angle de diffusion des bielles & \\
\hline$\tau_{c p}$ & Contrainte de cisaillement & {$[\mathrm{Pa}]$} \\
\hline$\tau_{\mathrm{IR}}$ & Contrainte de cisaillement dans la colonne fictive surmontant l'inclusion ou la colonne & {$[\mathrm{Pa}]$} \\
\hline$\tau_{\mathrm{s}}$ & Contrainte de cisaillement hors emprise de l'inclusion ou de la colonne & [Pa] \\
\hline
\end{tabular}




\section{Acronymes}

ACC

AGAP

ANR

ASIRI

CB

CBR

$\mathrm{CCH}$

CT

DLC

DR

DTU

ELS

ELU

ERP

F

IR

LT

MASW

NS

OPM

PLU

PPR

PSV

[Q]

QP

$\mathrm{R}$

[R]

SOLCYP

ZIG
Accidentel

Assurance qualité des prestations de services en Géophysique APpliquées

Agence Nationale de la Recherche

Amélioration des Sols par Inclusions Rigides (Projet national) (www.irex-asiri.fr)

Colonne Ballastée

Californian Bearing Ratio

Code de la Construction et de l'Habitation

Court Terme

Cas de charges pour la conception (Design Load Case, Norme NF EN 61-400)

Demande de Renseignements

Document Technique Unifié

Etat Limite de Service

Etat Limite Ultime

Etablissement Recevant du Public

Fondamental

Inclusion Rigide

Long Terme

Multichannel Analysis of Surface Wave

Non Soulevé

Optimum Proctor Modifié

Plan Local d’Urbanisme

Plan de Prévention des Risques

Profil Sismique Vertical

Sondage/essai fournissant une information qualitative. A n'envisager qu'en complément d'autres essais (cf. USG « Recommandations sur les investigations minimales »)

Quasi Permanent

Rare

Sondage/essai particulièrement bien adapté. A envisager en priorité (cf. USG « Recommandations sur les investigations minimales »)

Sollicitations CYcliques des Pieux (Projet national et ANR) www.pnsolcyp.org

Zone d'Influence Géotechnique 


\section{Contexte}

On rappelle que, conformément à l'avant-propos national de l'Eurocode 7 partie 1 et du § A.P.1 (1), pendant « la période de transition nécessaire à l'achèvement de cet ensemble de normes européennes..., les membres du CEN ont l'autorisation de maintenir leurs propres normes nationales adoptées antérieurement ».

Par ailleurs, suivant l'Eurocode 7 (NF EN 1997-1, 2005, section 2 ( Bases du calcul géotechnique ) $§ 2.1$ (21)), les éoliennes « ouvrages très grands ou inhabituels » appartiennent à la catégorie géotechnique 3 « qui devraient normalement faire appel à des dispositions ou règles alternatives à celles de cette norme »).

Ces recommandations concernent le dimensionnement et le contrôle des fondations d'éoliennes et peuvent s'inscrire dans le cadre de ces dispositions ou règles alternatives.

Les actions menées pour la conception, le calcul, l'exécution et le contrôle des fondations d'éoliennes, s'appuient en premier lieu sur les règles en vigueur, et des dispositions complémentaires ont été incluses dans le présent texte pour tenir compte des spécificités particulières de ce type d'ouvrage.

Ces recommandations feront l'objet de mises à jour en fonction des retours d'expérience, cela compte tenu des progrès attendus dans la connaissance des sollicitations réelles du sol (par le biais de l'instrumentation d'éoliennes), du comportement des fondations sous sollicitations cycliques (avancement du projet SOLCYP), de la mise en application des Eurocodes et du projet ASIRI sur les Inclusions rigides.

1

\section{Introduction}

-

\section{Définitions et types d'éoliennes}

Les éoliennes, dispositifs permettant de transformer l'énergie du vent en énergie mécanique, sont généralement classées en différentes catégories. Ce classement s'effectue principalement en fonction de leur hauteur, de leur lieu d'implantation et du diamètre de leur rotor lié à la puissance produite.

\section{(1)}

\section{Éoliennes essentiellement terrestre de plus de $12 \mathrm{~m}$}

Ces recommandations s'appliquent aux éoliennes à axe horizontal, à configuration amont (pales situées du côté de la tour exposée au vent) ou aval, implantées sur le domaine terrestre (éoliennes on-shore). Elles s'appliquent aux éoliennes à destination industrielle dont l'axe de rotation du rotor est situé à plus de $12 \mathrm{~m}$ audessus de la plate-forme. En revanche, l'utilisation de ces recommandations ne se justifie pas pour la vérification des éoliennes domestiques de moins de $12 \mathrm{~m}$.

Les termes ( éolienne », utilisé préférentiellement, ( aérogénérateur » ou « turbine à vent » désignent une machine constituée des éléments suivants :

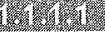

\section{Le rotor}

Le rotor est composé d'un ensemble de pales et de l'arbre primaire. Le rotor est relié à l'arbre secondaire de la nacelle par le moyeu. C'est l'élément qui reçoit directement l'énergie du vent.

\section{W.}

\section{La nacelle}

La nacelle est l'ensemble situé au sommet de l'éolienne qui abrite l'installation de génération de l'énergie électrique et ses périphériques (génératrice, multiplicateur de vitesse, frein, refroidisseurs...).

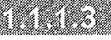

\section{Le mât ou tour}

Le mât est la partie supportant la nacelle et le rotor. Il les place à une hauteur suffisante pour obtenir les meilleures conditions de vent et pour assurer le mouvement libre des pales. On distingue les mâts haubanés, les mâts en treillis (pour de petites éoliennes) et les mâts tubulaires. Seul le cas des éoliennes à mât tubulaire est traité dans ce document.

L'ensemble formé du mât et du système de fondation est communément désigné par structure de support.

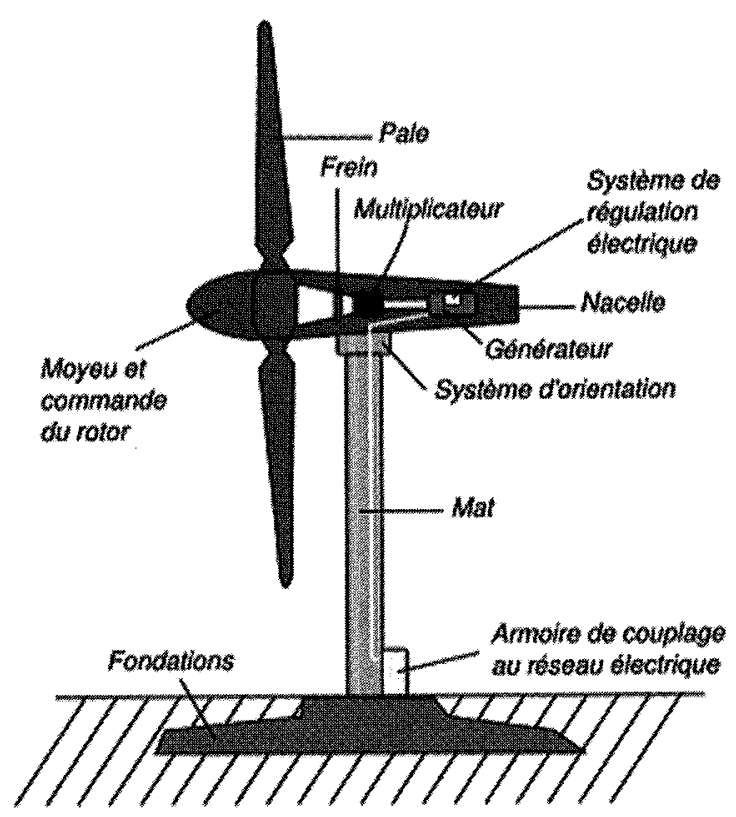

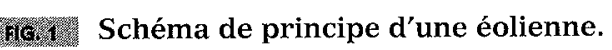

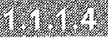

\section{Le système de fondation}

Le système de fondation comprend en partie supérieure le socle qui assure la liaison avec le mât et les éléments de fondations transmettant les charges au sol. Dans ce document, les éléments de fondations envisagés sont les suivants :

- fondations superficielles (embase-poids ; cf. § 5.2) ;

- fondations superficielles sur sol renforcé par des colonnes ballastées (cf. § 5.3); 
- fondations superficielles sur sol renforcé par des inclusions rigides (cf. § 5.4);

- fondations profondes de type pieux (cf. § 5.5) ;

- fondations ( ( mixtes ) ou fondations ( composites ) (cf. $§ 5.6$ ).

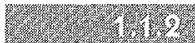

\section{Éolienne offshore}

Les éoliennes offshore doivent faire l'objet d'études spécifiques pour tenir compte des efforts particuliers agissant sur la structure (houle, chocs de navires, glace...) et des conditions géotechniques spécifiques au milieu marin. Elles n'entrent pas dans le cadre de ces recommandations.

\section{Prentis}

\section{Éoliennes de moins de $12 \mathrm{~m}$}

Les éoliennes de moins de $12 \mathrm{~m}$ ne rentrent pas dans le cadre des recommandations données dans ce document. En effet, ce type d'éoliennes n'obéit pas aux mêmes formalités vis-à-vis du code de l'urbanisme, même si l'obtention d'un permis de construire et le respect de certaines dispositions ou lois sont de vigueur.

Ces éoliennes sont, pour la majorité des cas, à usage domestique. Compte tenu de leurs dimensions, elles s'apparentent plus à des dispositifs comme les lampadaires, les candélabres, les panneaux...

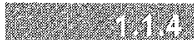

\section{Éoliennes repliables haubanées}

Les éoliennes repliables haubanées visent à limiter les dégâts sur la structure en cas de tempêtes, d'ouragans ou de tornades. On les trouve plus particulièrement dans les zones fortement touchées par ces phénomènes climatiques (Antilles, La Réunion...). Elles devront faire l'objet d'études spécifiques.

\section{1)}

\section{Parcs, fermes, groupes, champs...}

On désigne indifféremment par (c champ éolien » ou ( centrale éolienne » ou encore ( ferme éolienne ») un ou plusieurs ensemble(s) d'éoliennes concentrées géographiquement et appartenant au même maître d'ouvrage ou fournisseur d'électricité.

On réservera la notion de (c parc éolien ) à la description de l'ensemble des « champs d'éoliennes » dans une région donnée.

Au sens des présentes recommandations, on désigne par ( groupe d'éoliennes ) un ensemble d'éoliennes construites dans une zone homogène du point de vue géologique et géotechnique (nature du sol, stratigraphie, propriétés mécaniques...).
$-1-$

EMBASE POIDS

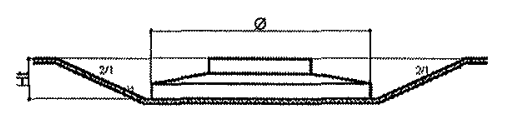

-3-

SUR INCLUSIONS RIGIDES

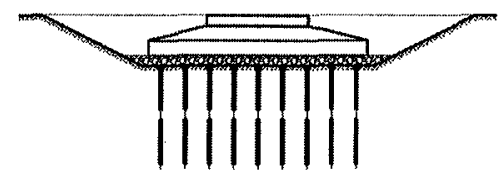

$-5-$

SUR FONDATIONS MIXTES

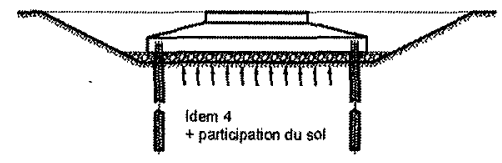

$-2-$ SUR COLONNES BALLASTEES

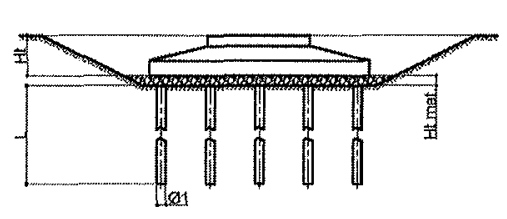

$-4 m$

SURPIEUX

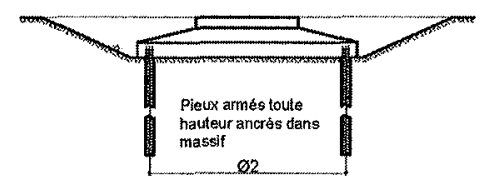

$-6-$ SUR FONDATIONS COMPOSITES

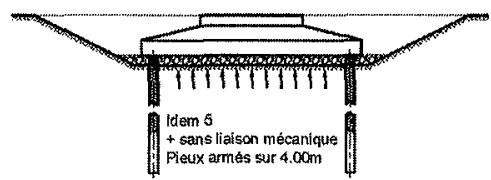




\section{Domaine d'application de ces recommandations}

Ce document s'adresse exclusivement aux éoliennes terrestre de plus de $12 \mathrm{~m}$, de taille moyenne (diamètre du rotor compris entre 12 et $60 \mathrm{~m}$ ) ou dites ( géantes ) (diamètres de rotor supérieurs à $60 \mathrm{~m}$ ).

Ce document ne s'applique pas directement :

- aux éoliennes offshore ;

- aux éoliennes haubanées.

Pour les éoliennes situées en zone sismique, une étude particulière doit être menée pour tenir compte de ce risque. Ce document ne traite pas de cette situation.

\section{1,3}

\section{Définitions d'intérêt général}

\section{1)}

\section{Données du site}

Les données du site sont les données environnementales, sismiques (non traitées dans ce document), sur le sol et le réseau électrique pour le site de l'éolienne. Les données du vent doivent être les statistiques d'échantillons sur 10 minutes, sauf indication contraire.

\section{5.}

\section{Conditions d'environnement}

Les conditions d'environnement que sont le vent, l'altitude, la température, l'humidité... peuvent affecter le comportement de l'éolienne.

\section{1.}

\section{Conditions extérieures}

Ce sont les facteurs affectant le fonctionnement d'une éolienne que sont les conditions d'environnement (température, neige, glace...) mais aussi les conditions d'état du réseau électrique. Les conditions de vent constituent le facteur extérieur principal dont il doit être tenu compte dans l'intégrité structurale.

Les propriétés du sol revêtent une importance significative dans la conception des fondations de l'éolienne.

\section{$16 x^{2}$}

\section{Contraintes d'environnement}

Au sens des présentes recommandations, les contraintes d'environnement résultent de la prise en compte des plans de prévention des risques naturels (PPR, annexés aux plans locaux d'urbanisme PLU), tels que définis par l'article L562-1 du code de l'Environnement et dont les objectifs sont de réduire la vulnérabilité des personnes et des biens. Les risques à évaluer comprennent; le risque d'inondation, les séismes (non traités dans ce document), les mouvements de terrain, les incendies de forêt, les avalanches...

\section{Référentiel}

La liste complète des textes et normes cités dans ce texte est en annexe F.

\section{1}

\section{Cadre réglementaire}

Depuis le $1^{\mathrm{er}}$ octobre 2008, l'article R111-38 du code de la Construction et de l'Habitation (CCH) est complété par un sixième point relatif au contrôle technique d'éoliennes dont la hauteur du mât et de la nacelle audessus du sol est supérieure à $12 \mathrm{~m}$.

Ces ouvrages s'inscrivent donc dans le cadre de la loi $\mathrm{n}^{\circ} 78-12$ du 4 janvier 1978 , relative à la responsabilité et à l'assurance dans le domaine de la construction modifiée par la loi $\mathrm{n}^{\circ}$ 2008-735 du 28 juillet 2008 dite ( loi Spinetta », et applicable sur tout le territoire français.

Commentaire : Ouvrages soumis obligatoirement au contrôle technique prévu à l'article L111-23 du CCH.

\section{3.}

\section{Les principes de la loi Spinetta}

La loi Spinetta comporte trois titres dont les principes fondamentaux sont:

\section{2.}

Titre I : présomption de responsabilité ( décennale ) pour tout constructeur d'un ouvrage

Est réputé constructeur d'un ouvrage :

- tout architecte, entrepreneur, technicien ou autre personne liée au maître de l'ouvrage par un contrat de louage d'ouvrage;

- toute personne qui vend, après achèvement, un ouvrage qu'elle a construit ou fait construire ;

- toute personne qui, bien qu'agissant en qualité de mandataire du propriétaire de l'ouvrage, accomplit une mission assimilable à celle d'un locateur d'ouvrage.

\section{1.}

Titre II : contrôle technique dans la réalisation des ouvrages

Le contrôleur technique intervient à la demande du maître de l'ouvrage et donne son avis à ce dernier sur les problèmes d'ordre technique « dans le cadre du contrat qui le lie à celui-ci ).

Cet avis porte notamment sur les problèmes qui concernent la solidité de l'ouvrage et la sécurité des personnes. Il formule à chaque stade de l'opération un examen critique sur les documents qui lui sont transmis et il s'assure que les vérifications techniques qui incombent aux constructeurs se déroulent de manière satisfaisante.

Le contrôle technique est rendu obligatoire pour certaines constructions, suivant l'article R111-38 du $\mathrm{CCH}$ et l'article 2 du décret $\mathrm{n}^{\circ}$ 2007-1327 du 11 sep- 
tembre 2007, dont les éoliennes « dont la hauteur du mât et de la nacelle au-dessus du sol est supérieure à 12 mètres $)$.

\section{1.}

\section{Titres III et IV : de l'assurance obligatoire des travaux de bâtiment et l'assurance des travaux de construction}

Toute personne physique ou morale, dont la responsabilité « décennale ) peut être engagée sur le fondement de la présomption établie par les articles 1792 et suivants du code civil, doit être couverte par une assurance.

Toute personne physique ou morale qui, agissant en qualité de propriétaire de l'ouvrage, fait réaliser des (c travaux de construction ), doit souscrire avant l'ouverture du chantier une assurance garantissant, en dehors de toute recherche des responsabilités, le paiement de la totalité des travaux de réparation de la totalité des dommages.

\section{2}

\section{Référentiel normatif}

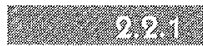 \\ Norme NF EN 61400-1 (juin 2006)}

La norme européenne NF EN 61400-1, 2005 présente les exigences de conception des éoliennes, notamment celles liées aux cas de charges. Elle n'est pas destinée à fixer des exigences pour les éoliennes installées en pleine mer, en particulier pour la structure de support (éléments d'une éolienne comprenant le mât et les fondations au sens du $\S 3.49$ de cette norme).

Le dimensionnement des fondations d'une éolienne doit permettre :

- de reprendre des efforts verticaux et horizontaux, statiques ou transitoires dus à l'éolienne elle-même, à son fonctionnement, aux conditions de vents et éventuellement aux séismes (non traité dans ce document) ;

- de n'engendrer que des tassements totaux et tassements différentiels compatibles avec le bon fonctionnement de l'éolienne.

La durée de vie prévue à la conception pour les classes I à III d'éoliennes (c'est-à-dire pour des vents ordinaires en site terrestre) doit être au moins égale à 20 ans ( 6.2 NF EN 61400-1).

Les éoliennes font l'objet d'une directive « Machine ». Elles sont assujetties à la directive européenne correspondante et soumises au marquage CE. La notion de machine est extensive et s'étend au mât de l'éolienne.

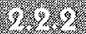

\section{Norme NFP 94-500 (décembre 2006)}

Le dimensionnement de la fondation s'appuie sur une investigation géotechnique adaptée, une bonne connaissance des efforts et une estimation correcte des contraintes et des tassements, qui doivent faire l'objet des missions d'ingénierie géotechnique telles que définies dans la norme NFP 94-500.
Les propriétés du sol sur un site proposé doivent être évaluées dans le cadre d'une étude géotechnique, en se référant aux normes et règlements de construction locaux disponibles.

\section{\%)}

\section{Norme de calculs des fondations}

Les fondations classiquement retenues sont de type superficiel ou profond. Un traitement ou un renforcement du sol de fondation se pratique également.

Les règles de calcul des fondations dépendent de leur nature (superficielle ou profonde). Les documents de référence en France sont actuellement :

- le fascicule 62 Titre V (MELT, 1993);

- les cahiers des charges particuliers validés ;

- les « Recommandations sur la conception, le calcul, l'exécution et le contrôle des colonnes ballastées sous bâtiments et sous ouvrages sensibles au tassement ) dites « Recommandations colonnes ballastées du CFMS, $2011)$;

- la note d'information technique de O. Combarieu, ( Calcul d'une fondation mixte semelle-pieux sous charge verticale centrée ».

Commentaire : Le document d'application nationale de l'Eurocode 0 (NF EN 1990-1/NA) propose un classement en catégories de durée d'utilisation de projet $(10,25,50$ et 100 ans) et en catégories géotechniques engendrant 3 familles de base de justification:

- expérience et reconnaissance géotechnique qualitative;

- reconnaissance géotechnique et calculs;

- reconnaissance géotechnique et calculs approfondis.

Les fondations des éoliennes entrent généralement dans la troisième catégorie.

\section{Ints}

\section{Norme NF P 03100 (septembre 95)}

L'activité de contrôle technique de la construction est exercée en conformité avec la norme française NF P 03100 relative au « Critères généraux pour la contribution du contrôle technique à la prévention des aléas techniques dans le domaine de la construction »).

La notion de contrôle technique implique l'existence d'un objet à contrôler et d'un référentiel par rapport auquel s'exerce ce contrôle.

Le référentiel est constitué par les dispositions techniques concernées par la mission de contrôle et figurant dans les documents relatifs au domaine de la construction :

- les normes françaises ;

- les documents techniques unifiés (DTU) ;

- les règles ou Recommandations professionnelles. 


\section{Cas de charge et sollicitations de calcul}

\section{1.}

\section{Introduction}

Les cas de charges élémentaires sont donnés par le constructeur. Ils reprennent certaines situations conceptuelles classées dans la norme NF EN 61400 (production électrique, production d'électricité plus survenance de la panne, immobilisation...).

Ces différents cas de charges sont calculés sur la base d'une durée de vie escomptée de la machine (pm : 20 ans soit environ 175000 heures).

\section{Analyse des cas de charge}

Les documents à prendre en considération sont :

- la norme NF EN 61-400;

- et en complément les cahiers des charges particuliers des constructeurs.

Les différents cas de charges doivent être transmis non pondérés.

\section{3.}

\section{Détermination des cas de charge utilisables vis-à-vis des fondations}

Le tableau 2 de l'article 7.4 (pages 34 et 35) de la NF EN 61-400 présente 22 cas de charge, qui intègrent ou non le poids du massif.

Commentaire : D'autres cas de charge doivent être pris en compte en complément s'ils sont relatifs à l'intégrité structurale dans la conception d'éoliennes spécifiques (haubanées, repliables...), mais ne font pas partie de ces Recommandations.

Il y a lieu de définir pour chacun des cas les pondérations à appliquer pour déterminer les sollicitations aux états limites ultimes (ELU) et de service (ELS).

\section{4}

\section{Sollicitations de calcul aux ELU/ELS}

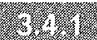

\section{Cas de charge déterminant vis-à-vis des fondations}

Ces cas de charges doivent être classés par référence aux sollicitations de calcul habituelles :

- ELS quasi permanent (QP) et ELS rare (R) ;

- ELU fondamental (F) et ELU accidentel (Acc).

Pour le dimensionnement des fondations, les cas de charge élémentaires (Design Load Case : DLC) pris en considération sont les suivants :

- situation conceptuelle $\mathrm{n}^{\circ} 1$ (production électrique) ;

- cas de charge 1.1, 1.3, 1.4 et 1.5 $\quad$ DLC $_{\text {Rare }}$

- cas de charge 1.2
- situation conceptuelle $n^{\circ} 2$ (production d'électricité + survenance de la panne);

- cas de charge 2.1,2.2 et $2.3 \quad$ DLC $_{\text {Rare }}$

- cas de charge $2.4 \quad$ Fatigue

- situation conceptuelle $n^{\circ} 5$ (arrêt d'urgence) ;

- cas de charge $5.1 \quad$ DLC $_{\text {Rare }}$

- situation conceptuelle $n^{\circ} 6$ (immobilisation [arrêt ou ralenti]) ;

- cas de charge 6.1 et 6.3

- cas de charge 6.2

- cas de charge 6.4

${ }_{\text {DLC }}$

DLC $_{\text {Acc }}$

Fatigue

- situation conceptuelle $\mathrm{n}^{\circ} 7$ (immobilisation et conditions de panne) ;
- cas de charge 7.1
DLC $_{\text {Acc }}$

Commentaires : A ce stade, et en attente des conclusions du projet national SOLCYP, les cas de charge ( fatigue ) ne sont pas à prendre en compte pour le dimensionnement du système de fondation vis-àvis du sol. Ils interviennent pour la vérification de la structure et du béton armé du massif.

Les constructeurs rajoutent parfois un cas « DLC 1.0》 considéré comme un $D L C_{Q P}$.

Les constructeurs doivent donner les cas les plus défavorables pour chacun des cas de charge $\mathrm{DLC}_{\mathrm{qp}}$ $D_{\text {Rare' }}, D_{\text {Acc }}$ et ( fatigue )).

\section{2.}

\section{Coefficients de pondération}

Hors cas de fatigue, pour définir les sollicitations de calcul aux ELS et ELU, il faut appliquer les coefficients de pondération du tableau I.

Commentaires : On rappelle que le torseur ramené à la base de la fondation est composé de $F_{z}$ l'effort vertical de compression, $H$ effort horizontal et $M$ moment de renversement. Ce torseur doit prendre en compte la présence ou l'absence d'eau; la pression résultante de l'eau est prise en compte si le niveau de la nappe est au-dessus de l'arase inférieure du massif.

On doit prendre en compte le facteur partiel de sécurité qui génère la situation de calcul la plus défavorable.

\section{5}

\section{Vérification des critères de dimensionnement}

\section{8.}

\section{Pourcentage de surface comprimée en cas de fondations superficielles}

Il s'agit de semelles considérées comme infiniment rigides, généralement circulaires.

On doit vérifier que les pourcentages de surface comprimée $\left(\mathrm{S}_{\text {comp }} / \mathrm{S}_{\text {sem }}\right)$ sont au moins ceux du tableau II.

\section{61.9}

\section{Critère de portance}

Les contraintes de calcul du sol permettent de justifier la fondation aux états limites de service (ELS) et aux états limites ultimes (ELU). 
TRBLAur Facteurs partiels de pondération sur sollicitations.

\begin{tabular}{|c|c|c|c|c|c|}
\hline \multirow{2}{*}{$\mathrm{DLC}_{\mathrm{QP}}$} & $E_{\text {Fond }}$ & 1,0 ou 1,35 & 1,8 & 1,8 & $1,125 \times 1,05$ \\
\hline & ELS $_{\text {perm }}$ & 1,0 & 1,0 & 1,0 & 1,0 \\
\hline \multirow{2}{*}{$\mathrm{DLC}_{\text {Rare }}$} & ELU ${ }_{\text {Fond }}$ & 1,0 ou 1,35 & 1,5 & 1,5 & $1,125 \times 1,05$ \\
\hline & ELS $_{\text {Rare }}$ & 1,0 & 1,0 & 1,0 & 1,0 \\
\hline $\mathrm{DLC}_{\mathrm{Acc}}$ & ELU $_{\mathrm{Acc}}$ & 0,9 ou 1,1 & 1,1 & 1,1 & 1,0 \\
\hline
\end{tabular}

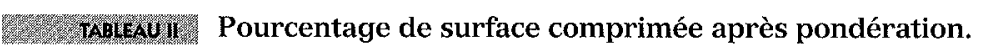

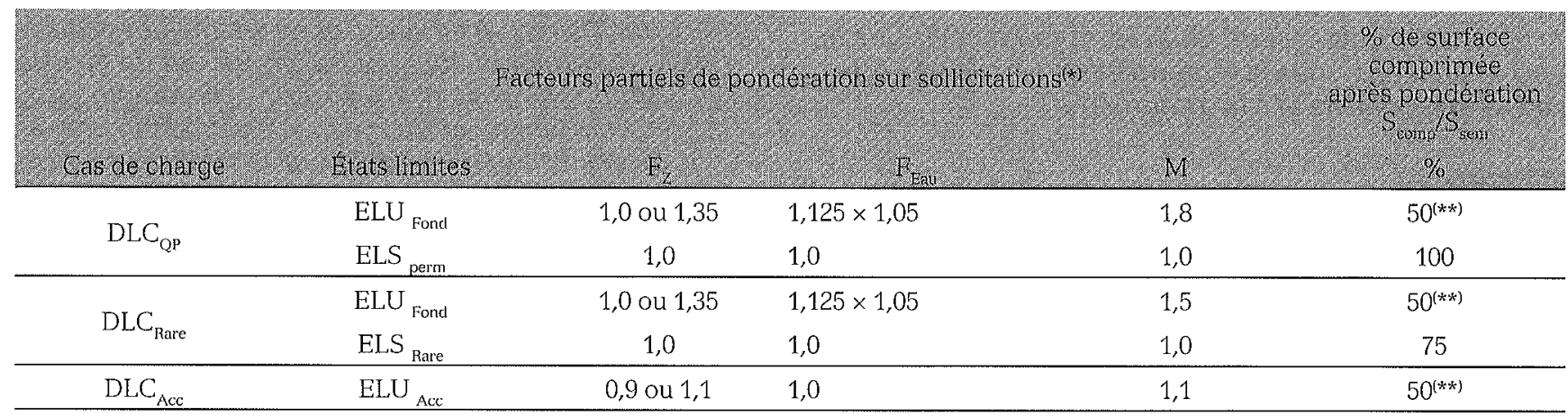

${ }^{(*)}$ Le facteur partiel à prendre en compte est celui qui génère la situation la plus défavorable.

${ }^{(* *)}$ Cette valeur est ramenée à $30 \%$ dans les types de sol suivants (cf. classification $\$ 4.6 .5 .4$ ).

\begin{tabular}{|c|c|c|c|}
\hline 1. ARBLAU III & \multicolumn{3}{|c|}{$\begin{array}{l}\text { Pourcentage de surface comprimée } \\
\text { après pondération; compléments } \\
\text { pour sols raides. }\end{array}$} \\
\hline \multicolumn{2}{|c|}{ Craies } & $\mathrm{B}^{+}+\mathrm{C}$ & 30 \\
\hline \multicolumn{2}{|c|}{ Marno, marno-calcaires } & $\mathrm{A}^{+}+\mathrm{B}$ & 30 \\
\hline \multicolumn{2}{|c|}{ Roches } & $\mathrm{A}^{+}+\mathrm{B}$ & 30 \\
\hline
\end{tabular}

- les sollicitations de calcul aux ELU déduites des cas de charge $D_{L C}, D_{O P} C_{\text {Rare }}$ et DLC ${ }_{A c c}$ (après pondération) permettent de déterminer la contrainte de calcul $\mathrm{q}_{\text {refELU }}(\mathrm{ELU}$ fondamental au sens du Fascicule 62-Titre V, MELT, 1993);

- les sollicitations de calcul aux ELS déduites des cas de charge DLC $\mathrm{OP}_{\mathrm{OP}}$ et DLC $\mathrm{DL}_{\mathrm{Rare}}$ (sans pondération) permettent de déterminer la contrainte de calcul $\mathrm{q}_{\text {reffis }}$ (ELS respectivement quasi permanent et rare au sens du Fascicule 62-Titre V, MELT, 1993).

Ces valeurs sont à comparer aux valeurs limites de résistances du sol établies dans le cadre d'une étude géotechnique.

On doit attirer l'attention sur le rôle majeur du paramètre ( $\%$ de surface comprimée ), qui, comme le montre la figure 3 pour des semelles circulaires, peut majorer la contrainte maximale à 8 fois la contrainte obtenue sous la même charge verticale supposée centrée quand ce pourcentage vaut $30 \%$.

Commentaire : La “ surface comprimée $S_{\text {comp }}$ ), utilisée ultérieurement et proposée dans la figure $n^{\circ} 6$, est celle obtenue en négligeant les parties soulevées. La surface de référence $S_{\text {ref }}$ est une " surface fictive de compression » utilisée dans les vérifications de portance globale. Ces deux surfaces sont définies en annexe $B$.

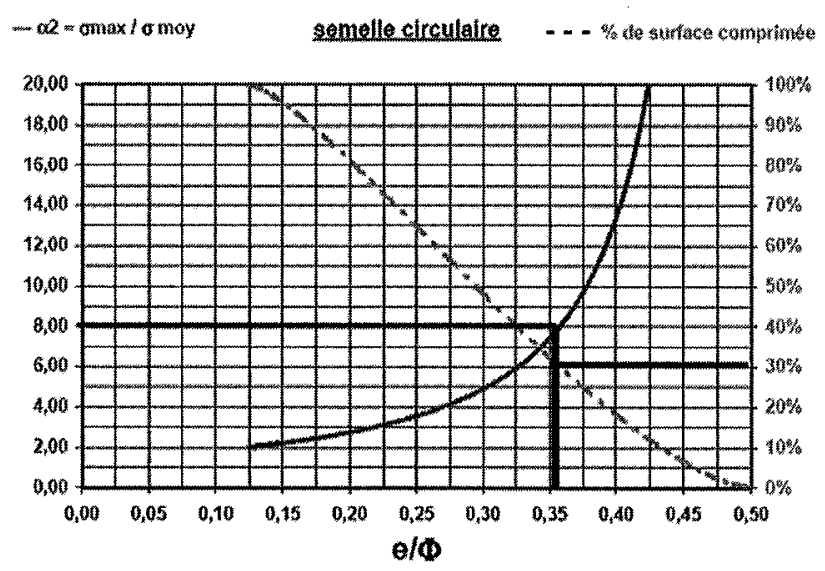

Corrélations entre excentricité, \% de surface comprimée $\mathrm{S}_{\text {comp }} / \mathrm{S}_{\text {sem }}$ et contrainte maximale.

Conformément aux règles en usage, on doit vérifier la contrainte de référence de calcul par rapport à la contrainte admissible aux ELS et aux ELU; cette contrainte de référence $q_{\text {ref }}$ vaut, en fonction des contraintes minimum $\sigma_{\min }$ et maximum $\sigma_{\max }$ sous la semelle :

$$
q_{\text {ref }}=\left(3 . \sigma_{\text {max }}+\sigma_{\text {min }}\right) / 4 \text { avec } \sigma_{\text {min }} \geq 0
$$

Cette contrainte de référence peut aussi se calculer pour une surface rectangulaire selon l'approche de Meyerhof, et pour une surface circulaire selon le modèle de la (c lunule ») (cf. Annexe B). 


\section{Modèle général}

A l’ELU, la contrainte de référence peut être calculée comme suit:

$$
\begin{gathered}
\sigma_{\text {moy }}=F_{\mathrm{zELU}} / \mathrm{S}_{\mathrm{sem}} ; \\
\alpha_{2}=\sigma_{\text {max }} / \sigma_{\text {moy }}
\end{gathered}
$$

(cf. courbe en trait plein de la figure 3) ;

$$
\mathrm{q}_{\text {ref }}=3 \cdot \alpha_{2} \cdot \sigma_{\text {moy }} / 4 \text { si } \sigma_{\text {min }}=0
$$$$
\mathrm{e}=\mathrm{M}_{\mathrm{ELU}} / \mathrm{F}_{\mathrm{zELU}}
$$

Exemple tiré de la figure $n^{\circ} 5: \mathrm{e} / \Phi=0,35>1 / 8$ $\%$ surface comprimée : $30 \% \mathrm{~S}_{\text {ref }}$

$$
\begin{gathered}
\alpha_{2}=\sigma_{\text {max }} / \sigma_{\text {moy }}=8, \text { et } \sigma_{\text {min }}=0 \\
\mathrm{q}_{\text {ref }}=3 \cdot \alpha_{2} \cdot \sigma_{\text {moy }} / 4=6 \sigma_{\text {moy }}
\end{gathered}
$$

\section{69}

\section{Modèle de la (c Iunule ) pour une surface circulaire}

La contrainte de référence peut être aussi calculée à partir de la méthode dite de la (c lunule ) telle que définie en annexe $B$.

- la surface de référence $S_{\text {ref }}$ est la zone hachurée de la figure en annexe B (lunule : limitée par deux arcs de cercles symétriques par rapport à un axe situé à $\mathrm{e}=\mathrm{M}_{\mathrm{ELU}} / \mathrm{F}_{\mathrm{zELU}}$ du centre de l'éolienne).

$-\mathrm{q}_{\mathrm{ref}}=\mathrm{F}_{\mathrm{zELU}} / \mathrm{S}_{\mathrm{ref}}$

Commentaire : Dans le cas d'une fondation circulaire, les deux calculs de $q_{\text {ref }}$ conduisent à des résultats très proches.

Il convient de vérifier que le système de fondations choisi est compatible avec la contrainte maximale.

\section{3.}

\section{Vérification au glissement}

On doit vérifier à l'ELU :

$$
\mathrm{H}_{\mathrm{ELU}}<\mathrm{F}_{\mathrm{zmin}} \cdot \tan \delta / \gamma_{\mathrm{s}}
$$

Commentaire : $\delta$ est l'angle de frottement entre la semelle et le sol qu'on assimile à l'angle de frottement $\varphi^{\prime}$ du sol, $\gamma_{s}$ est un coefficient de sécurité valant 1,2 sous combinaisons fondamentales et 1,1 sous combinaisons accidentelles et $F_{z \min }$ est le minimum de compression transmis au sol par la semelle. Pour les éléments de fondation coulés en place il faut utiliser l'angle à l'état critique du matériau. La norme soutènements fait observer en complément que $\phi^{\prime}$ est souvent supérieur à $\phi_{\text {critique }}^{\prime}$ Pour les éléments préfabriqués, c'est 2/3 de l'angle à l'état critique.

La valeur de $\varphi^{\prime}$ à prendre en compte est établie dans le cadre d'une étude géotechnique.

En général, on ne prend pas en compte la butée du sol, ni la cohésion du sol.

Commentaire : Les efforts horizontaux en sous-face de la semelle sont donc égaux à ceux appliqués en tête de semelle. Néanmoins, dans le cas où le massif est coulé en pleine fouille, on pourra faire participer la butée de la semelle jusqu'à une valeur déterminée lors de l'étude géotechnique, qu'on limitera cependant à $30 \%$ de la valeur maximale de la poussée passive.

\section{Critère de rotation à long terme (LT)}

Le constructeur fournit généralement une valeur de critère de rotation limite $(\mathrm{en} \mathrm{mm} / \mathrm{m})$ à l'ELS. Cette valeur ne doit jamais être dépassée durant la vie de l'ouvrage. Elle prend en compte les déformations permanentes sous l'effet de vent normal (il pourra être retenu les valeurs issues de la combinaison $\mathrm{DLC}_{\mathrm{QP}}$ avec des caractéristiques (c long terme )), mais aussi des effets « court terme ) correspondants à des vents plus importants issus des cas de charge DLC ${ }_{\text {rare }}$ ou DLC Acc $_{\text {(calculés sur }}$ la base des caractéristiques ( court terme »).

On désigne par coefficient de raideur en rotation (exprimé en $\mathrm{MNm} / \mathrm{rad}$ ou un multiple) le rapport du moment de rotation $\mathrm{M}_{\mathrm{xy}}$ appliqué à la fondation sur la rotation $\varphi$ de celle-ci.

$$
\varphi=M_{x y} / K_{. \varphi}
$$

$\mathrm{K}_{\varphi}(\mathrm{en} \mathrm{MNm} / \mathrm{rad})$ avec

$\mathrm{K}_{\text {का , }, \mathrm{T}}$ coefficient de raideur en rotation « à long terme se evalué pour $\mathrm{DLC}_{\mathrm{QP}}$ :

- calculé à partir des formules habituelles de la mécanique des sols (laboratoire, essais pressiométriques ou pénétrométriques) ;

$K_{\varphi C T}$ coefficient de raideur en rotation « à court terme s) non pondéré évalué pour $\mathrm{DLC}_{\mathrm{rare}}-\mathrm{DLC}_{\mathrm{OP}}$ (ou pour $\mathrm{DLC}_{\mathrm{Acc}}-\mathrm{DLC}_{\mathrm{QP}}$ en conformité avec le cahier des charges du constructeur):

- calculé à partir des formules de géodynamique et des modules de cisaillement $G$ (cf. § 4.6.3.2);

- calculé à partir de modèles analytiques numériques ; - ou, à défaut, calculé à partir des valeurs LT de la mécanique des sols dans le domaine des déformations de l'ordre de $10^{-2}$ et en multipliant cette valeur par 2.

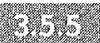

\section{Critère de raideur en rotation $\mathrm{K}_{\varphi \text { dyn }}$}

\section{6) (6)}

\section{Raideur en rotation}

Les constructeurs imposent une valeur minimale de la raideur en rotation à petite déformation (de $10^{-5}$ à $10^{-3}$ ) ( $K_{\text {pdyn }}$ ) pour éviter les phénomènes de couplage avec les parties mécaniques de la machine.

Cette valeur est à prendre en compte sous tous les cas en fonctionnement de la machine :

$-\mathrm{DLC}_{\mathrm{QP}}$

- et certains DLC $\mathrm{C}_{\text {Rare }}$ en conformité avec les cahiers des charges des constructeurs (cas des situations conceptuelles $n^{\circ} 1$ et $n^{\circ} 2$ de la norme NF EN 61400 ).

Les calculs de la raideur en rotation nécessitent de connaître pour un massif de fondation :

- ses dimensions (diamètre, aire...) ;

- le pourcentage de surface comprimée ;

- et par couches de sol, les courbes de variation des modules d'élasticité $E$ et des modules de cisaillement $\mathrm{G}$ en fonction de la distorsion $\gamma$, le coefficient de Poisson $v$.

Dans la mesure où le sol reste entièrement comprimé sous la totalité du massif de fondation et si on reste dans le domaine élastique, on pourra utiliser les coefficients de raideur $\mathrm{K}_{\varphi \mathrm{NS}}$ (cf. tableau $\mathrm{n}^{\circ} 16$ du paragra- 
phe 5.2.3.3.1.1) sur la base d'un module de cisaillement $G$ pris dans la plage de déformation adéquate.

Commentaire : Dans le cas où le sol ne reste pas entièrement comprimé, il y a lieu de prendre un coefficient réducteur $\beta_{1}=\mathrm{K}_{\text {op }} / \mathrm{K}_{\text {tps }}$ appliqué à la raideur en rotation en fonction du pourcentage de surface comprimée $\left(\mathrm{S}_{\text {comp }} / \mathrm{S}_{\mathrm{sem}}\right)$. Le calcul de $\beta_{1}$ se fait par analogie à la résistance des matériaux avec une solution implicite par itérations successives.

En première approche, les valeurs de $\boldsymbol{\beta}_{1}$ sont représentées en fonction du rapport $M_{x y} / F_{z}\left(M_{x y}\right.$ non pondéré et $F_{z}$ non pondérée) et du diametre de la fondation $\Phi=2$ r dans le graphique suivant.

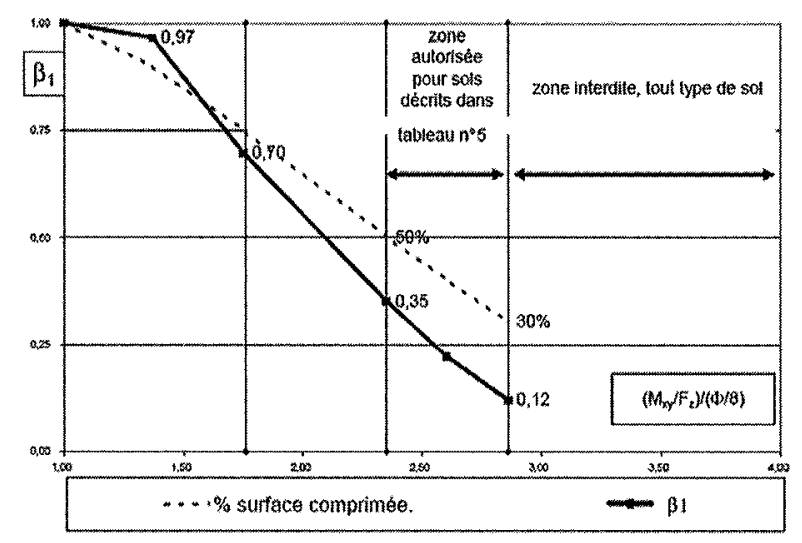

Valeurs du coefficient réducteur $\beta 1$ appliqué à la raideur en rotation.

Exemple d'application :

$$
\begin{gathered}
\left(\mathrm{M}_{\mathrm{xy}} / \mathrm{F}_{z}\right) /(\operatorname{diam} / 8)=2,3 \\
\rightarrow \% \text { comprimée }=50 \% \\
\text { et } \beta_{1}=0,35, \rightarrow \mathrm{K}_{\varphi}=0,35 \mathrm{~K}_{\mathrm{\varphi NS}}
\end{gathered}
$$

\section{6 .}

\section{Raideurs (( statique ) et (( dynamique )}

Pour des distorsions $\gamma$ de l'ordre $10^{-2}$ à $10^{-3}$, les raideurs en rotation sont usuellement qualifiées comme ( statiques ).

Pour des distorsions $\gamma$ de l'ordre $10^{-6}$ à $10^{-4}$, les raideurs en rotation sont usuellement qualifiées comme (c dynamiques ).

Dans le cadre de ces recommandations, les raideurs en rotation « statiques » sont à utiliser pour la vérification des tassements et des déformations, et les raideurs en rotation (c dynamiques ) permettent de s'assurer de l'absence de phénomène de couplage entre le sol, la fondation et la machine.

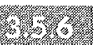

\section{Critères de raideur en déplacement}

Ils sont parfois fixés par le constructeur, et sont liés aux raideurs horizontales $\left(\mathrm{K}_{x^{\prime}} \mathrm{K}_{\mathrm{y}}\right)$, et verticales $\mathrm{K}_{\mathrm{z}}$

Commentaire: On désigne par coefficient de raideur vertical ou horizontal (exprimé en $\mathrm{N} / \mathrm{m}$ ou un multiple) le rapport de la force verticale $F_{z}$ ou horizontal $H$ appliquée à la fondation sur le déplacement vertical ou horizontal $w$ de celle-ci.
Les raideurs calculées doivent être supérieures aux valeurs imposées par le constructeur.

$$
4
$$

\section{Géotechnique et paramètres de dimensionnement}

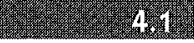 \\ Introduction}

On rappelle que l'enchaînement des missions types d'ingénierie géotechnique est défini par la norme NF P 94-500.

Celui-ci comprend notamment au stade ( études ) : - une étude géotechnique d'avant-projet (G12) qui identifie les aléas majeurs, s'assure de la bonne adéquation entre la nature et la profondeur des terrains rencontrés avec ceux proposés par les cartes géologiques et estime les caractéristiques mécaniques de chaque couche. Elle préconise un ou plusieurs types de fondations ;

- une étude géotechnique de projet (G2).

Si la mission préalable (G12) a identifié des aléas majeurs, elle doit définir les dispositions pour en limiter les conséquences (par exemple, réalisation d'une étude de microgravimétrie préconisant un déplacement de l'éolienne ou une injection des karsts situés sous son emprise). De plus, dans le cadre de cette mission, le géotechnicien doit vérifier que la fondation respecte les critères géotechniques ainsi que ceux mentionnés dans le cahier des charges du fabricant d'éoliennes et doit définir également des modules dans les différentes plages de déformations.

Il comprend aussi au stade ( exécution » :

- l'étude et le suivi géotechniques d'exécution (mission G3)

- la supervision géotechnique d'exécution (mission G4).

\section{2}

\section{Éléments à fournir au géotechnicien}

Le maître d'ouvrage, assisté par son maître d'œuvre, doit fournir au géotechnicien (conformément au $\$ 3.47$ (( Données du site de la NF EN 61-400 ), juin 2005) :

- l'implantation du projet ;

- le nivellement du projet;

- le plan topographique ;

- le plan des réseaux existants et la liste des concessionnaires pouvant être concernés (Demande de renseignements, décret 91-1147);

- les plans particuliers des risques (PPR);

- les critères d'environnement ;

- les différentes descentes de charge (conformément au chapitre 3 des présentes recommandations) ;

- les exigences imposées en termes de :

- tassement ( $W$ ) et déformations ( $\varepsilon$ ), distorsion

( $\gamma)$, raideur,

- contraintes requises du sol (le cas échéant). 


\section{Données géotechniques}

Les études géotechniques successives permettent de préciser :

- le périmètre géotechnique au sens de la norme NF P 94 500, 2006 (Zone d'influence géotechnique - ZIG -, stabilité de pente, etc.) ;

- la géologie et la stratigraphie ;

- l'hydrologie et hydrogéologie du site ;

- les niveaux d'eau ;

- l'agressivité (eau et sol);

- un modèle géotechnique décrivant les différentes couches à prendre en compte, et définissant par couche (liste non exhaustive)

- l'épaisseur,

- la nature des terrains (cf. tableau n ${ }^{\circ} 12$ du $\$$ 4.6.5.4,

et norme ISO 14688-1 et 2 et 14689-1 et 2),

- les perméabilités, si nécessaire,

- une pression limite moyenne ou une valeur au pé-

nétromètre statique moyenne,

- les modules (cf. \$ 4.6),

$\mathrm{E}_{\mathrm{yst}}$ pour une déformation $\boldsymbol{\varepsilon}$ de l'ordre de $10^{-2}$ [souvent dénommés ( modules d'Young )]

$E$ ou $G$ pour des déformations $\varepsilon$ comprises entre $10^{-3}$ et $10^{-4}$

- le coefficient de Poisson $v$,

- les dispositions constructives ;

- les hypothèses géotechniques de dimensionnement les types de fondations et leurs justifications (cf. § 4.6).

\section{4.}

\section{Reconnaissance minimale hors zone d'anomalies}

On définit l'anomalie comme étant par exemple (liste non exhaustive) :

- zone de dissolution, carrière, ordure ménagère, karst, remblais de forte épaisseur, zone d'instabilité, zone glissée..

On définit le programme des reconnaissances : - par zones homogènes au sens géologique et en tenant compte du nombre d'éoliennes ;

- par éolienne.

\section{4.}

Par groupe (ou zone) homogène au sens géologique

\section{4.}

\section{Définition}

Une zone homogène au sens géologique est un site pour lequel la nature et la stratigraphie géologiques sont considérées homogènes.

Un groupe d'éoliennes représente le nombre d'éoliennes à construire dans une zone homogène.

\section{1.}

Carottage avec prélèvements

(NF EN ISO 22475-1)

Les prélèvements minimaux sont réalisés à raison de un par faciès géotechnique; ils permettent :
- l'identification des matériaux (prélèvements remaniés);

- la caractérisation des propriétés géomécaniques (prélèvements intacts) : mesure de $\varphi^{\prime}$ et $c^{\prime}$ selon norme NF P 94074.

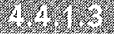

\section{Piézomètre (NF EN ISO 22475-1)}

Un piézomètre permet la réalisation de mesures ponctuelles dans le temps. Le suivi des mesures est à réaliser le plus tôt possible avant le démarrage de la construction.

Les conditions de réalisation devront être conformes à la norme. Nous rappelons qu'il faut éviter :

- le colmatage des crépines (filtre d'enrobage et remblai de graviers) ;

- les venues d'eau d'origine météorique en tête (massif béton en tête coulé en pleine fouille et tête de protection).

La durée minimale de relevés sera de 12 mois avec une intervention minimale mensuelle.

\section{Whes}

\section{Essais géophysiques}

Ils sont décrits dans le document de l'AGAP «Code de bonne pratique en géophysique appliquée ». On utilisera plus particulièrement les essais de type : CrossHole, MASW, sismocône ou équivalent.

Ils doivent permettre :

- de mesurer au moins $\mathrm{V}_{\mathrm{s}^{\prime}}$ voire $\mathrm{V}_{\mathrm{p}}$;

- et donc de déterminer le module de cisaillement $G_{\text {max }}$ à $10^{-6}$ de distorsion, voire le coefficient de Poisson $v$.

\section{$44^{2}$}

\section{Nombre minimal et nature des sondages}

Le tableau IV résume le nombre et le type de sondages à réaliser.

\section{(1)}

\section{Par éolienne}

De plus, par éolienne, on réalise au minimum 4 sondages ( 1 au centre et 3 en périphérie [entre 5 à $15 \mathrm{~m}$ du centre]) dont :

- 1 sondage in situ [R] au centre ;

- soit pressiométrique selon la norme NF P 94-110-1 avec essais pressiométriques tous les mètres;

- soit au pénétromètre statique $\left(q_{c^{\prime}} f_{s^{\prime}}, R_{f}\right)$, selon la norme NF P 94-113.

Commentaire : Il est nécessaire de souligner que des fluctuations de la nappe peuvent occasionner une baisse significative des valeurs mesurées dans la tranche possiblement non saturée à l'époque des essais qui deviendrait saturée ensuite.

- trois sondages [Q] à choisir en fonction de la nature des sols pour vérifier en particulier l'homogénéité (profondeur...) des sols sous l'emprise de la fondation ;

- soit au pénétromètre statique $\left(\mathrm{q}_{\mathrm{c}^{\prime}} \mathrm{f}_{\mathrm{s}^{\prime}}, \mathrm{R}_{\mathrm{f}}\right)$, selon la norme NF P 94-113;

- soit pressiométrique selon la norme NF P 94-110-1 avec essais pressiométriques tous les mètres, 


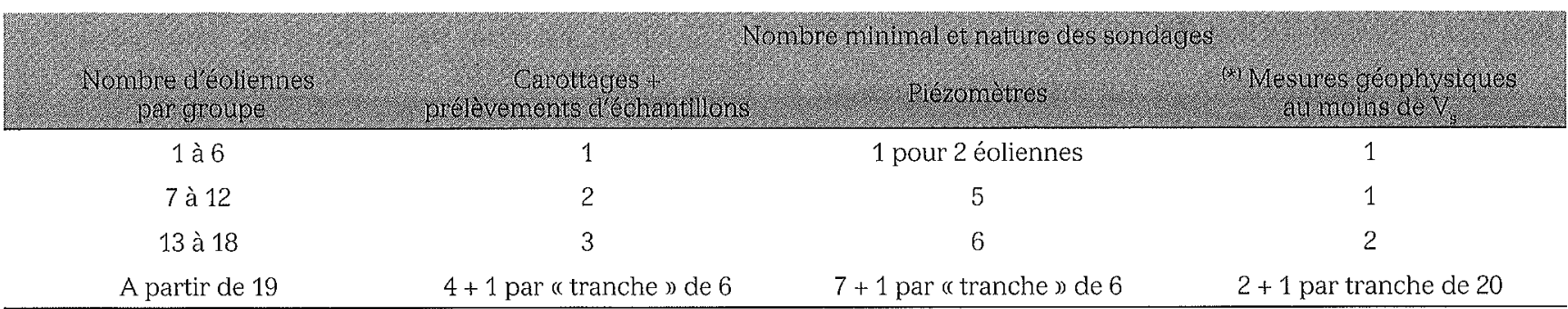

${ }^{(*)}$ selon les domaines de classement de sol (cf. \$ 4.6.5)

- soit destructif avec enregistrement de paramètres [cf. Reiffsteck et al. (2010)] ;

- soit au pénétromètre dynamique selon la norme NF

P 94-114 ou au SPT selon la norme NF P 94-116;

- soit à la pelle.

Commentaires : On trouvera la définition de $[R]$ et [Q] dans le texte USG « Recommandations sur les investigations géotechniques pour la construction (Le Moniteur $n^{\circ} 5325$ du 16 décembre 2005) 》, avec pour mémoire :

- [R] : sondage/essai particulièrement bien adapté. A envisager en priorité ;

- [Q] : sondage/essai fournissant une information qualitative. An'envisager qu'en complément d'autres essais.

On préconise les sondages à la pelle en cas de substratum rocheux rencontré à faible profondeur.

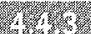

\section{Profondeur des investigations}

\section{Hy.}

\section{Sondage carotté et sondage réalisé au centre de chaque éolienne}

La reconnaissance de sol doit permettre de déterminer les caractéristiques de sol sur une profondeur égale ou supérieure à celle sur laquelle les contraintes) induites par le massif sont encore sensibles et engendrent des déformations significatives.

On pourra ainsi limiter la profondeur de reconnaissance sous le massif de la manière suivante :

- pour les massifs de type fondation superficielle de diamètre $\varnothing$ (cf. figure 2), la plus petite des deux valeurs ;

- 1,5 fois le diamètre théorique du massif : $1,5 \varnothing$;

Commentaire : Cette limitation à $1,5 \varnothing$ (et non pas $8 \varnothing$ selon les errements) suppose qu'au-delà, et en adéquation avec la carte géologique, les sols ont des caractéristiques supérieures ou égales à celles mesurées au-dessus et qu'on peut ainsi négliger les déformations de ceux-ci. Si cette hypothèse n'est pas avérée, il faut prolonger la reconnaissance.

- la profondeur à laquelle les modules de sols sont suffisants pour ne plus engendrer des déformations significatives du massif, profondeur majorée de $5 \mathrm{~m}$.

Commentaires : Pour des éoliennes de 80 à $100 \mathrm{~m}$ de hauteur, une valeur de module $E_{y s t}$ supérieure à $100 \mathrm{MPa}$ peut être considérée.

Dans le cas d'essai pressiométrique, une valeur de module $E_{m}$ supérieure à 1000 fois l'augmentation des contraintes peut aussi être considérée.
- pour des fondations profondes de diamètre $\Phi 1$ jusqu'à la plus grande des trois valeurs suivantes sous la pointe:
$7 \varnothing 1$
5 mètres,
$\varnothing / 2$.

Commentaire : Cette dernière condition peut permettre de répondre à la problématique de l'effet de groupe.

- pour des fondations mixtes ou composites, et pour des sols renforcés par inclusions rigides ou colonnes ballastées de diamètre $\Phi 1$, on prend l'enveloppe des deux cas précédents.

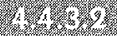

\section{Autres sondages en périphérie}

La reconnaissance de sol doit permettre de vérifier l'homogénéité (profondeur, nature...) des sols sous l'emprise de la fondation. Elle peut donc être limitée en profondeur au toit du substratum sain ou de l'horizon de fondation.

\section{5}

\section{En présence d'anomalie}

En complément des prescriptions du paragraphe précédent, et en conformité avec la norme NF P94-500 pour identifier les aléas majeurs et importants, il convient d'approfondir les investigations définies ci-avant par une analyse au cas par cas permettant de s'assurer que les investigations intéressent la totalité de l'épaisseur de sol concernée par les anomalies et ayant encore une influence sur l'ouvrage.

\section{6}

\section{Modèle géotechnique}

\section{6)}

\section{Pour des déformations comprises entre $10^{-2}$ et $10^{-3}$}

Les paramètres principaux de calcul à fournir pour les couches de sol qui intéressent le projet sont les suivants :

- les domaines de classement des couches de sol (cf. § 4.6.5.) ;

- les niveaux d'eau à prendre en compte dans les calculs ; 
- les caractéristiques géomécaniques de rupture définies à partir des essais in situ et des essais de laboratoire (cf. annexe F) par exemple ;

- la pression limite nette $p_{1}^{*}$,

- la résistance de pointe au pénétromètre statique $\mathrm{q}_{c^{\prime}}$

- les résistances de cisaillement : $\varphi^{\prime}$ et $c^{\prime}$,

- les paramètres de déformation du sol permettant de calculer les tassements et les rotations du massif en fonction du niveau de déformation du sol, à savoir :

- les valeurs du modules de déformation $E$, et du module de cisaillement $\mathrm{G}$ (cf. figure 9), en fonction du niveau de déformation $\varepsilon$ des cas étudiés $\left(10^{-2}>\varepsilon>\right.$ $\left.10^{-3}\right)$,

- les valeurs du coefficient de Poisson v.

Commentaires : Pour les sols non saturés, les coefficients de Poisson v à court terme et v à long terme sont identiques et sont compris entre 0,20 et 0,35.

Pour les sols saturés :

- à long terme, $v$ doit être compris entre 0,20 et 0,35 ;

- et à court terme, v est généralement compris entre 0,30 et 0,45 .

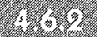

\section{Pour des déformations comprises entre $10^{-3}$ et $10^{-4}$}

Sous sollicitations cycliques, certains types de sol peuvent subir une dégradation de leurs caractéristiques mécaniques liées par exemple à une montée progressive des surpressions interstitielles, ou à une attrition du sol. Le géotechnicien de l'opération devra définir l'éventualité de ce risque.

La reconnaissance de sol à partir des essais in situ traditionnels doit être complétée par une campagne plus détaillée permettant de mesurer (cf. Fig. 5) :

- les paramètres à très faible déformation du sol (cf. § 4.4.1.4 : essais géophysiques);

- les paramètres à faible déformation du sol à partir par exemple d'essais au laboratoire (type essai à la colonne résonante ou essai au triaxial cyclique).

et ainsi d'estimer la courbe complète $\mathrm{E} / \mathrm{E}_{\max }$ et/ou $\mathrm{G} / \mathrm{G}_{\max }$ en fonction de $\varepsilon$ et/ou de $\gamma$.

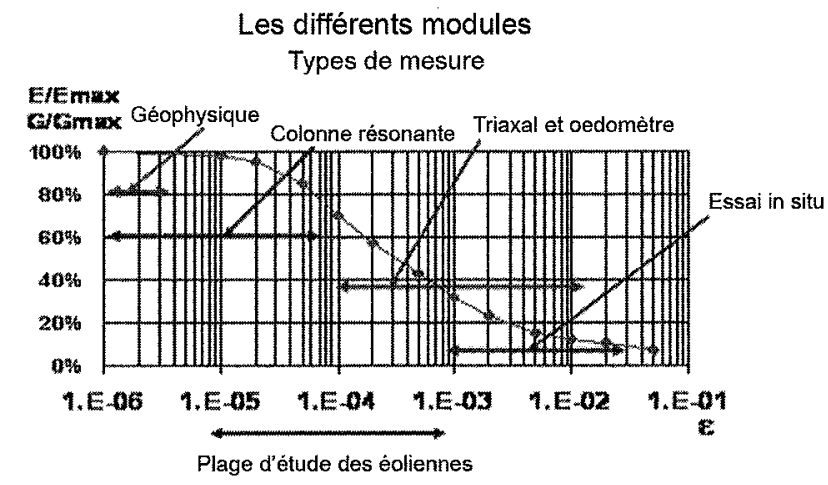

Hes Schéma indicatif de principe (d'après F. Durand, CFMS, oct. 2009).

Commentaires : Ce schéma n'est que représentatif des différentes plages de déformations et ne doit en aucun cas être utilisé en dimensionnement. On pourra consulter en annexe $C$ les courbes de dégradation de $G$ en fonction de la distorsion pour des matériaux. argileux et granulaires.
Les vitesses d'ondes de cisaillement et de compression déterminées à partir de mesures géophysiques pourront utilement être comparées aux valeurs moyennes habituelles données dans le tableau 5.2.1 de la norme NF P 06013 [dites Règles PS 92] rappelées dans l'annexe C (chapitre C.2).

Tout ou partie de ces paramètres est nécessaire pour, entre autres, le calcul des différents coefficients de raideur verticale, horizontale et en rotation.

\section{4.}

\section{Données géotechniques permettant le dimensionnement de la semelle}

\subsection{6.}

\section{Portance}

Il faut connaître sous la base du massif les pressions limites ( $\left.p_{1}\right)$ ou les valeurs pénétrométriques statiques ( $\left.q_{c}\right)$.

Pour calculer la portance, on doit déterminer une pression limite équivalente ( $\left.p_{\text {le }}{ }^{*}\right)$ ou une valeur pénétrométrique équivalente $\left(\right.$ $\left.\mathrm{q}_{\mathrm{ce}}\right)$ calculée à partir des valeurs précédentes mesurées sur une hauteur de $1.5 \varnothing$ sous la semelle (cf. annexe E.2 du fascicule 62-Titre V et $\$ 3.2 .2$ de la norme NF P11-211 [DTU 13.11])

Cette façon de déterminer les caractéristiques moyennes des sols intéressés sur $1.5 \varnothing$ sous la semelle ne nous paraît pas pleinement compatible pour les cas de fondations de grande dimension, en particulier parce qu'elle limite la valeur de calcul à 1,5 fois la plus faible des valeurs mesurées de pression limite. Nous proposons donc ci-après une méthode de calcul de $q_{c e}$ et de $\mathrm{p}_{\mathrm{le}}$ appropriée à ce type d'ouvrage permettant de mieux pondérer le « poids » d'une valeur faible en fonction de sa profondeur par rapport à la base du massif. Les valeurs de $\mathrm{p}_{\mathrm{le}}$ et/ou de $\mathrm{q}_{\mathrm{ce}}$ en fonction d'une gamme de diamètres $\Phi$ sont établies dans la cadre d'une étude géotechnique.

\section{1.}

Méthode proposée de calcul de $p_{l e}$ adaptée aux semelles de grande dimension

Pour tenir compte des variations de mesures de $p_{1}$ sur 1,5 $\Phi$, on propose la méthode de calcul suivante de $\mathrm{p}_{\mathrm{le}}$, basée sur la formule de la semelle fictive :

- à chaque niveau $\alpha_{i} \Phi\left(\alpha_{i}\right.$ compris entre 0 et 1,5$)$ de mesure de pression limite $\mathrm{p}_{\mathrm{l},}$, on utilisera la formule de la semelle fictive avec une diffusion de $1 \mathrm{H} / 2 \mathrm{~V}$ pour déterminer la pression limite de calcul $p_{\text {Ici }}$ telle que $p_{\text {lci }}=p_{\text {li }}$ * $\left[\left(1+\alpha_{i}\right)^{2}\right]$

$-\mathrm{p}_{\mathrm{le}}=\min \operatorname{des} \mathrm{p}_{\mathrm{li}}{ }^{*}\left[\left(1+\alpha_{\mathrm{i}}\right)^{2}\right]$ ainsi calculées sur une profondeur 1,5 $\Phi$

Commentaires : $\alpha \Phi$ correspond au toit de la tranche (cf. exemple en annexe D). Cette méthode présente l'avantage de pondérer le ( poids » d'une valeur faible en fonction de sa profondeur par rapport à la base du massif.

Dans le cas d'une semelle partiellement comprimée, on limitera ces calculs à 1,5 $b^{\prime}$, avec $b^{\prime}$ défini sur la figure suivante et en remplaçant $\varnothing$ par $b^{\prime}$ dans les équations 10 et 11. 


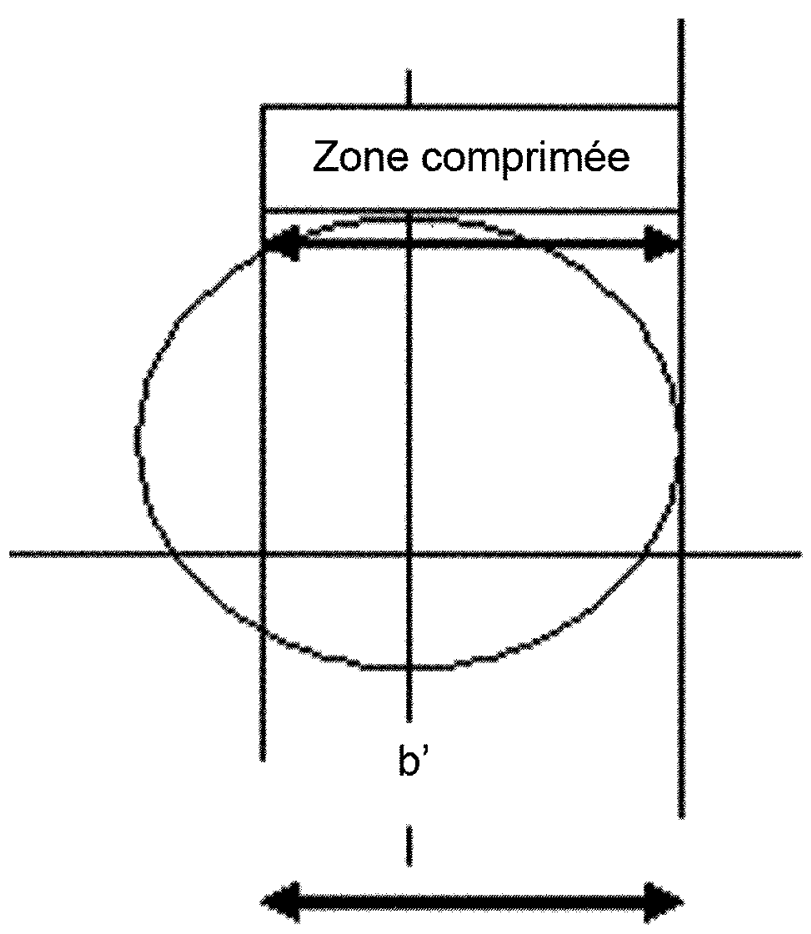

16.6 Définition de la dimension de la zone comprimée : b' (cf. annexe B)
Commentaire : Dans le cas d'un sol homogène caractérisé par des variations des pressions limites entre une valeur max $p_{l \max }$ et une valeur min $p_{\text {Imin }}$ telle que $p_{\text {Imax }} / p_{\text {Imin }}<2$, on peut simplifier cette methode de calcul, et déterminer $p_{t}{ }^{*}$ en prenant une moyenne géométrique et en limitant le résultat à $1,5 p_{\text {Imin }}$.

\section{(3) 6 (1)}

Méthode proposée de calcul de $q_{c e}$

Pour tenir compte des variations de $q_{c}$ sur $1,5 \Phi$, on se réfère à la méthode de calcul du Fascicule 62-Titre V (MELT 1993 et Annexe E2) :

- on calcule la moyenne arithmétique des $q_{c}$ sur 1,5 $\mathbf{\Phi}$; - puis on écrête les valeurs de $q_{c}$ à 1,3 fois cette moyenne ;

- puis on recalcule la moyenne des valeurs écrêtées qu'on retient comme valeur de $\mathrm{q}_{\mathrm{ce}}$.

Commentaire : Dans le cas d'une semelle partiellement comprimée, on limitera ces calculs à 1,5 b' (cf. figure 5 et annexe B).

En cas de couche molle d'épaisseur métrique, on appliquera la méthode de la semelle fictive en limitant $\mathrm{q}_{\mathrm{ce}}$ à la valeur ainsi mesurée :

- à chaque niveau $\alpha_{i} \Phi\left(\alpha_{i}\right.$ compris entre 0 et 1,5$)$ de mesure de valeur pénétrométrique $\mathrm{q}_{\mathrm{ci}}$ on utilisera la formule de la semelle fictive avec une diffusion de $1 \mathrm{H} / 2 \mathrm{~V}$ pour déterminer la valeur pénétrométrique de calcul $\mathrm{q}_{\mathrm{cci}}$ telle que $\mathrm{q}_{\mathrm{cci}}=\mathrm{q}_{\mathrm{cj}}{ }^{*}\left[\left(1+\alpha_{\mathrm{i}}\right)^{2}\right]$;

$-\mathrm{q}_{\mathrm{ce}}=\min \operatorname{des} \mathrm{q}_{\mathrm{ci}} \cdot\left[\left(1+\alpha_{\mathrm{i}}\right)^{2}\right]$ ainsi calculées sur une profondeur 1,5 $\Phi$.

\section{\%}

\section{Déformation}

\subsection{8.}

Entre $10^{-3}$ et $10^{-2}$

La valeur du tassement $w$ est déterminée à partir des méthodes habituelles de la Mécanique des Sols :

- les méthodes à partir des essais de laboratoire : il s'agit essentiellement de l'essai cedométrique surtout utilisé pour les sols fins cohérents et saturés;

- les méthodes à partir des essais en place : essai pres siométrique Ménard, essai de pénétration statique CPT, très utilisées notamment pour les sols pulvérulents.

Commentaires : Dans le cas de l'essai pressiométrique Ménard, on se référera à l'Annexe F2 du Fascicule 62-Titre $V$ (MELT, 1993) pour l'évaluation du tassement $w$ et à l'Annexe F3 de ce Fascicule pour l'évaluation du module de réaction verticale $K_{v s}$

Dans le cas de l'essai au pénétromètre statique, la résistance de pointe $q_{c}$ est reliée au module odométrique $E_{\text {oed }}$ et au module d'Young $E_{y s t}$ (cf. $\$ 4.6 .5$ ) par les relations suivantes:

$$
\mathrm{E}_{\text {oed }}=\alpha_{1} \mathrm{q}_{\mathrm{c}}
$$

(pour des déformations de l'ordre de $10^{-2}$ )

$$
\mathrm{E}_{\text {oed }}=\mathrm{E}_{\mathrm{yst}}(1-v) /[(1+v)(1-2 v)]
$$

Le tableau $V$ propose des fourchettes de valeurs de $\alpha_{1}$ pour différents types de sol normalement consolidé et différentes valeurs de $q_{c}$. (Frank, 1996).

Dans le domaine de déformations statiques comprises entre $10^{-3}$ et $10^{-2}$, il faut donc connaître :

- soit les caractéristiques pressiométriques permettant;

\section{TABAuY Valeur de $\alpha 1$ pour différents types de sol et

\begin{tabular}{|c|c|c|c|}
\hline Argile peu plastique & $<0,7$ & & 3 à 8 \\
\hline Argile peu plastique & $<2,0$ & & 2 à 5 \\
\hline Argile peu plastique & $>2,0$ & & 1 à 2 \\
\hline Limon peu plastique & $<2,0$ & & 3 à 6 \\
\hline Limon peu plastique & $>2,0$ & & 1 à 2 \\
\hline Argile très plastique & $<2,0$ & & 2 à 6 \\
\hline Limon très plastique & $>2,0$ & & 1 à 2 \\
\hline Limon très organique & $<1,2$ & & 2 à 8 \\
\hline $\begin{array}{l}\text { Tourbe et argile } \\
\text { très organique }\end{array}$ & $<0,7$ & $50-100 \%$ & 1,5 à 4,0 \\
\hline $\begin{array}{l}\text { Tourbe et argile } \\
\text { très organique }\end{array}$ & $<0,7$ & $100-200 \%$ & 1,0 à 1,5 \\
\hline $\begin{array}{l}\text { Tourbe et argile } \\
\text { très organique }\end{array}$ & $<0,7$ & $>200$ & $<1$ \\
\hline Craie & 2 à 3 & & 2 à 4 \\
\hline Craie & $>3$ & & 1,5 à 3 \\
\hline Sable & $<5$ & & 2 \\
\hline Sable & $>10$ & & 1,5 \\
\hline
\end{tabular} différentes valeurs de $q_{c}$.}


- de définir en fonction de la nature du sol le coefficient rhéologique $\alpha$ et de calculer le module sphérique $\mathrm{E}_{\mathrm{c}}$ et le module déviatorique $\mathrm{E}_{\mathrm{d}}$ selon les usages en vigueur,

- de calculer le tassement sphérique $\mathrm{w}_{\mathrm{c}^{\prime}}$ le tassement déviatorique $\mathrm{w}_{\mathrm{d}}$,

- et le tassement total w,

- et enfin de calculer une raideur $K_{v s}=q / w$ statique à court terme (CT) et à long terme (LT),

- soit les caractéristiques au pénétromètre statique $q_{c^{\prime}}$ le rapport $\alpha_{1}=E_{\text {oed }} / q_{\mathcal{c}^{\prime}}$ permettant :

- de calculer sous une charge apportant une contrainte q le tassement total $w$,

- et, enfin, de calculer une raideur $\mathrm{K}_{\mathrm{vs}}=\mathrm{q} / \mathrm{w}$ statique

à CT et à LT,

- soit les caractéristiques mesurées en laboratoire.

Ces différentes valeurs de $\mathrm{K}_{\mathrm{vs}}$ à CT et à LT sont établies dans la cadre d'une étude géotechnique.

\section{Why}

Entre $10^{-3}$ et $10^{-5}$

Pour un disque rigide reposant sur un milieu élastique homogène semi-indéfini, l'extension de la solution donnée en 1885 par Boussinesq pour le cas d'une charge verticale centrée aboutit dans le cas de chargement complexe aux modules suivants :

$$
\begin{array}{r}
\mathrm{k}_{\mathrm{v}}=4 \mathrm{G} /(\pi \mathrm{r}[1-\mathrm{v}]) \text { en } \mathrm{N} / \mathrm{m}^{2} / \mathrm{m} \\
\mathrm{K}_{v}=4 \mathrm{Gr} /(1-v) \text { en } \mathrm{N} / \mathrm{m} \\
\mathrm{K}_{\varphi}=8 \mathrm{Gr}^{3} /[3(1-v)] \text { en } \mathrm{MNm} / \mathrm{rad}
\end{array}
$$

Ce modèle élastique est valable pour une semelle soumise à un moment sans décollement, pour des actions de courte durée d'application ne provoquant ni consolidation, ni fluage.

Commentaire : Ne s'applique strictement qu'au cas d'une semelle soumise à un moment sans décollement : $r$ peut être identifié au rayon du disque. Si on a un effort vertical excentré, la largeur de la zone comprimée est inférieure à $2 r$. Il faut donc adopter un rayon équivalent $r^{*}$ à définir (a priori égal à celui du disque de même inertie que la zone comprimée).

Les valeurs de $\mathrm{G}$ dans le domaine de déformation considéré sont établies dans la cadre d'une étude géotechnique.

\section{6.}

\section{Données permettant de dimensionner les pieux, les inclusions rigides ou les colonnes ballastées}

Conformément aux usages (Fascicule 62-Titre V, MELT, 1993) ( Recommandations colonnes ballastées », CFMS, 2011) pour justifier la portance et pour calculer les tassements des pieux, des inclusions rigides ou des colonnes ballastées (de longueur L et de diamètre $\varnothing_{1}$ ), et la rotation de ce massif, il faut reconnaître au droit de l'ouvrage le sol sur une profondeur égale à $\mathrm{h}+\mathrm{L}+$ $\max \left(5 \mathrm{~m} ; 7 \varnothing_{1} ; \varnothing / 2\right)$ (cf. $\$ 5.4 .1$ ).

Commentaire : La reconnaissance sera adaptée en cas de risque d'anomalie connue (karst, carrière...).

Les études géotechniques successives doivent permettre de préciser une coupe type, avec par couche :

- la nature des terrains ;
- la pression limite moyenne ou la valeur au pénétromètre statique caractéristique permettant de calculer le frottement et l'étreinte latérale pour les colonnes ballastées;

- une valeur maximale de la pression limite équivalente $\left(\mathrm{p}_{\mathrm{le}}{ }^{*}\right)$ ou de la valeur au pénétromètre statique $\left(\mathrm{q}_{\mathrm{ce}}\right)$ permettant de calculer le terme de pointe ;

- la valeur moyenne du module pressiométrique pour calculer la mobilisation du frottement ;

- la valeur du coefficient rhéologique $\alpha$, pour calculer les raideurs horizontales du sol ;

- la valeur du module pressiométrique moyen sous la pointe pour calculer la mobilisation de la pointe.

\section{(1.}

\section{Les domaines de classement de sol}

En fonction des valeurs des modules de déformation (module d'Young) (c statique habituel ) $\mathrm{E}_{\mathrm{yst}}$ (déformation comprise entre $10^{-3}$ et $10^{-2}$ ) et des essais in situ (pressiomètre et/ou pénétromètre statique), il est possible de différencier trois domaines d'études pour un sol équivalent sur une épaisseur de 1,5 $\Phi$

\section{2.}

\section{Domaine $\mathrm{n}^{\circ} 1: \mathrm{E}_{\mathrm{yst}}<15 \mathrm{MPa}$}

Dans ce domaine, a priori, il n'est pas possible d'envisager des fondations superficielles sans aménagement particulier :

- si la couche directement au contact du massif se trouve dans le domaine $n^{\circ} 1$, les caractéristiques de sol de cette couche ne permettent pas la réalisation de fondations superficielles compatibles avec les critères de déformation et de rotation prescrits par les constructeurs dans leur cahier des charges particulier. Dans ce cas, les fondations profondes s'imposent. On pourra également s'orienter vers une adaptation du système de fondation superficielle par l'utilisation de substitution ou de renforcement de sol ;

- si une couche de sol appartenant au domaine $n^{0} 1$ se situe suffisamment en profondeur avec une faible épaisseur, on pourra éventuellement se ramener au domaine $n^{\circ} 2$ moyennant une étude spécifique.

\section{1.}

\section{Domaine $\mathrm{n}^{\circ} 2$ : $15 \mathrm{MPa} \leq$ Eyst $\leq 50 \mathrm{MPa}$}

Dans le cas d'un multicouche sur une profondeur égale à 1,5 fois le diamètre du massif, si une des couches se trouve dans le domaine $n^{\circ} 2$ et si aucune couche ne se situe dans le domaine $n^{\circ} 1$, le projet se situe dans le domaine $n^{\circ} 2$.

Si une couche de sol appartenant au domaine $n^{\circ} 1$ se situe suffisamment en profondeur avec une faible épaisseur, on pourra éventuellement se ramener au domaine $n^{\circ} 2$ moyennant une étude spécifique.

La seule définition du module de déformation « statique habituel » $\mathrm{E}_{\mathrm{yst}}$ ne permet pas de retenir en l'état un système de fondation superficielle.

Toutefois, un principe de fondation superficielle n'est pas exclu.

- pour cela, la reconnaissance de sol à partir des essais in situ traditionnels doit être complétée par une campagne plus détaillée $(\$ 4.6 .2)$ permettant de mesu- 
rer les paramètres à très faible déformation du sol et ainsi d'estimer la courbe complète $E / E_{\max }$ et/ou $G / G_{\max }$ en fonction de $\varepsilon$ et/ou de $\gamma$;

- on peut également s'orienter vers un renforcement de sol.

Commentaire : Dans ce cadre de fondations superfcielles sur sol renforcé ou substitué, et dans le cadre de fondation mixte ou composite, on pourra s'affranchir de la campagne détaillée du § 4.6.2.

Dans le cadre d'un prédimensionnement, on pourra utiliser la corrélation suivante entre les modules ( statiques ) et les modules maximum à très faible déformation [de l'ordre de $10^{-6}$ ] ( dits dynamiques » (cf. $\$ 3.5 .5 .2)$ :

$\mathrm{G}_{\max }=10 \mathrm{G}$, avec $\mathrm{G}$ module « statique » pour des déformations à $10^{-2}$

$E_{\max }=10 E_{y^{\prime} t^{\prime}}$ avec $E_{\text {yst }}$ module (c statique ) pour des dêformations à $10^{-2}$

Dans le cadre de corrélations à partir de l'essai pressiométrique, on peut retenir :

$$
\mathrm{G}_{\max }=\left(6 \text { à 8) } \mathrm{E}_{\mathrm{m}}\right.
$$

Par défaut, pour les taux de déformation considérés pour les éoliennes $\left(\varepsilon \approx 10^{-3}\right.$ à $\left.10^{-4}\right)$, on peut considérer que :

$\mathrm{G}_{\grave{1} 10}{ }^{-4} / \mathrm{G}_{\max }=0,33$ pour les matériaux argileux et compacts

$\mathrm{G}_{\grave{a} 10}{ }^{-4} / \mathrm{G}_{\mathrm{max}}=0,50$ pour les matériaux sablo-graveleux compacts (et roches altérées).

Pour les autres matériaux, une interpolation est possible.

En prédimensionnement, la prise en compte de valeurs plus favorables que celles obtenues par les corrélations précédentes doit faire l'objet des essais décrits dans le $4^{\mathrm{e}}$ paragraphe du chapitre 4.6.5.2.

\section{T.}

\section{Domaine $n^{\circ} 3: \mathrm{E}_{\mathrm{yst}}>50 \mathrm{MPa}$}

Un principe de fondation superficielle de l'éolienne est tout à fait envisageable et la reconnaissance de sol fournissant des modules de déformation « statiques habituels » $\mathrm{E}_{\text {yst }}$ est suffisante.

- pour que le projet soit dans le domaine $n^{\circ} 3$, toutes les couches sur une profondeur égale à 1,5 fois le diamètre du massif doivent se situer dans le domaine $n^{\circ} 3$;

- si une couche de sol appartenant au domaine $n^{\circ} 2$ se situe suffisamment en profondeur avec une faible épaisseur, on pourra éventuellement se ramener au domaine $n^{\circ} 3$ moyennant une étude spécifique.

\section{6.}

\section{Classement}

En reprenant les types de sols tirés du Fascicule 62-Titre V (MELT, 1993), on propose le classement des domaines décrits ci-dessus synthétisés dans le tableau « Synthèse des domaines d'études ».

Dans la pratique, et sur une hauteur égale à 1,5 fois la largeur du massif $\Phi$, il convient de définir les différentes couches de sols de caractéristiques géologiques et mécaniques homogènes.

Les caractéristiques moyennes de ces différentes couches de sol sont déterminées de la façon suivante :

$q_{c E q}$ et $E_{m E q}$ sont obtenus en établissant la moyenne harmonique (respectivement de $\mathrm{q}_{c}$ et de $\mathrm{E}_{\mathrm{m}}$ ) sur la hauteur de la couche considérée, et limités à 1,5 fois la plus faible des valeurs mesurées.

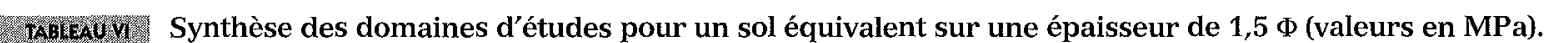

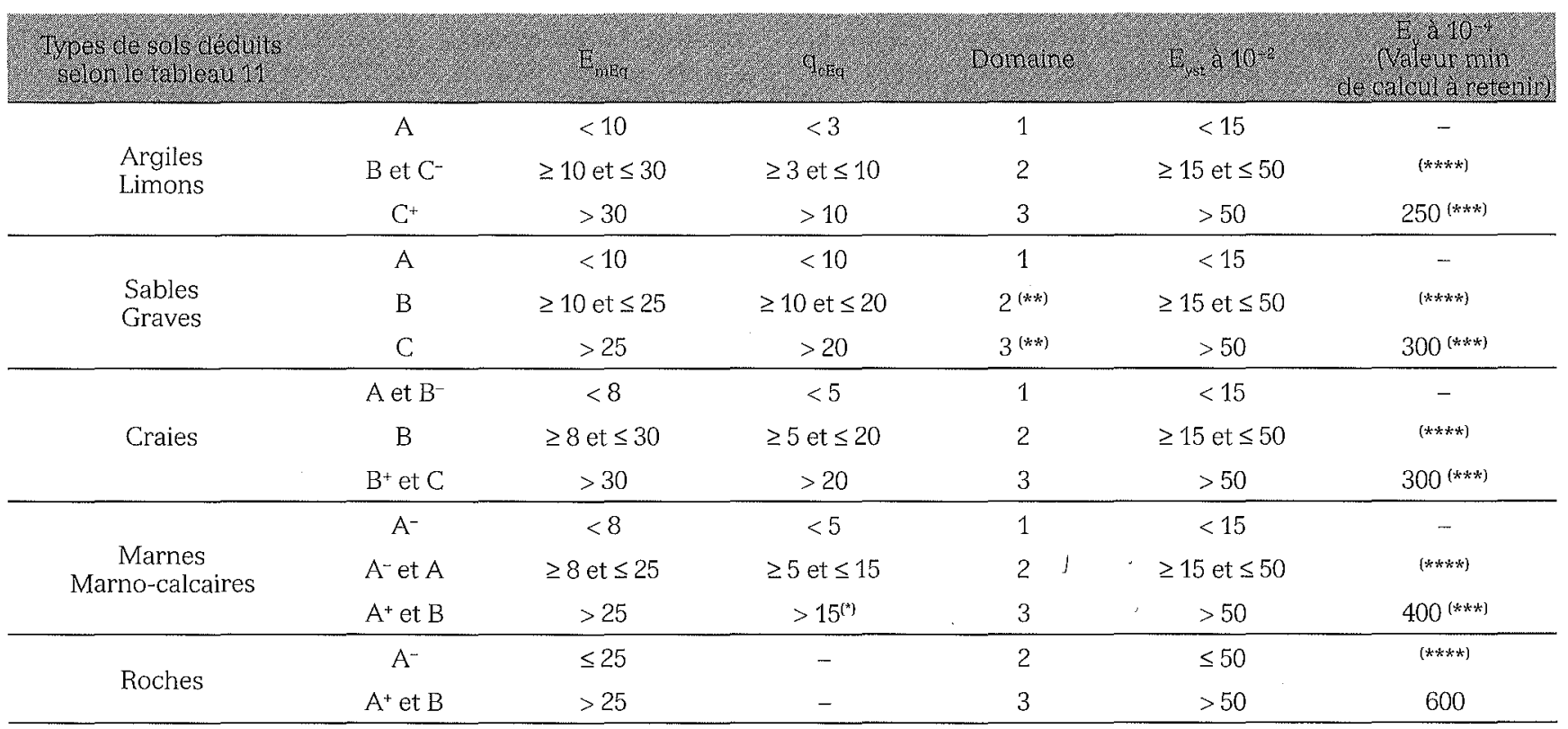

(*) Plus essais complémerlaires si sefus prétraluré.

${ }^{(* *)}$ Plus étude de liquéfaction sous sollicitations cycliques à réaliser si $\mathrm{D}_{10}$ (diamètre du passant à $10 \%$ ) $<2$ mm, Norme NF P 06-013-PS 92 Article 9.122. ${ }_{(* \star *)}$ Déterminées à partir de corrélations habituelles. ${ }_{(* * * *)}^{(* *)}$ Valeurs à définir lor's de la reconnaissance complémentaire.

$\mathrm{A}^{-}, \mathrm{A}^{+}, \mathrm{B}^{-}, \mathrm{B}^{+}, \mathrm{C}^{-}, \mathrm{C}^{+}$: catégories supplémentaires à celles proposées dans le Fascicule 62-Titre $\mathrm{V}$

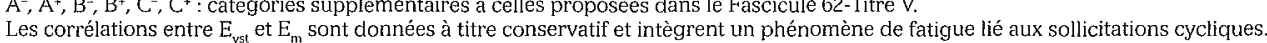




\section{Les différents types de fondation}

\section{1}

\section{Généralités}

\subsection{1}

\section{Préambule}

Un massif de fondation d'éolienne a généralement une forme polygonale assimilable à un massif circulaire de même surface et de diamètre $\varnothing$. Sa base est généralement horizontale et se situe à une profondeur d'encastrement $h$ par rapport au niveau de la surface.

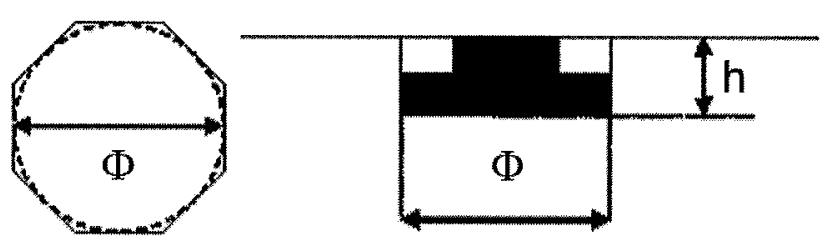

IC. I Massif de fondation.

Il peut être fondé (cf. § 1.1.1.4) :

- soit sans renforcement du sol : il s'agit « d’embase poids 》 (§5.2);

- soit sur sols renforcé de colonnes ballastées [CB] (§ 5.3) ;

- soit sur sol renforcé d'inclusions rigides [IR] (\$ 5.4) ;

- soit sur pieux : il s'agit de fondations profondes $(\S 5.5)$;

- soit en « fondations mixtes » ( ou en fondations composites 》 (§5.6).

Commentaires : Les solutions de renforcement par colonnes mixtes (de type inclusion rigide surmontée d'une tête en colonnes ballastées) doivent vérifier et respecter simultanément les recommandations et les dispositions des colonnes ballastées et des Inclusions Rigides telles qu'explicitées dans les $\$ 5.3$ et 5.4 .

Pour les sols dont les caractéristiques mécaniques sont susceptibles d'évoluer significativement dans le temps (décharges, remblais sous-consolidés, vases et tourbes...), on s'orientera préférentiellement vers des solutions de types fondations profondes, ou embases-poids après substitution de ces sols.

\section{6.}

\section{Dispositions constructives communes}

\section{3.}

\section{Vis-à-vis de nappe d'eau}

En présence de nappe, on prend toujours l'effet de celle-ci.

Les niveaux d'eau à prendre en compte sont établis dans la cadre d'une mission géotechnique.

\section{3.}

\section{Vis-à-vis de l'eau de surface}

En cas de risque d'accumulation d'eau et de saturation du terrain jusqu'à un niveau supérieur à la base de la fondation, on prend l'effet de l'eau sauf à pouvoir justifier d'un drainage gravitaire pérenne.

Commentaire : Les niveaux d'eau à prendre en tenant compte de la topographie du site, de la stratigraphie, des perméabilités des différentes couches et de la pluviométrie de la zone, sont établis dans la cadre d'une étude géotechnique.

\section{tris 9}

\section{Vis-à-vis de l'altération du fond de fouille}

Après réception du fond de fouille par le géotechnicien dans le cadre d'une supervision géotechnique d'exécution (étape 3 de la norme NF P94-500), on prend les dispositions nécessaires pour protéger le fond de fouille en cours de travaux.

Selon la solution de fondation utilisée, on réalise alors soit un béton de propreté, soit une couche de protection de type plate-forme de travail ou couche de support de fondation.

On prendra des dispositions constructives nécessaires pour que cette couche de protection ne soit pas contaminée par le sol support en fond de fouille (géotextile, couche anti-contaminante...).

\section{3.}

Vis-à-vis de la mise en butée des sols

On se référera au paragraphe 3.5.3.

\section{texists.}

\section{Longueur minimum du renforcement (CB, IR ou pieux)}

Sauf justifications particulières, la longueur minimale des renforcements est la hauteur des terrains situés en domaine $n^{\circ} 1$ et/ou $n^{\circ} 2$.

\section{1.}

\section{Vérifications et contrôles communs}

Les dispositions des règlements en vigueur s'appliquent et sont complétées par les suivantes.

\section{3.}

\section{Béton de massif}

Les documents du marché précisent le nombre et la nature des prélèvements. Les fréquences recommandées sont au minimum les suivants :

- de 1 prélèvement pour $100 \mathrm{~m}^{3}$ de béton mis en place ;

- et de 1 prélèvement par éolienne;

- cela à raison de 6 éprouvettes par prélèvement.

Les caractéristiques du béton devront être conformes à la NF EN 206. 


\section{then}

Vis-à-vis de la portance et de l'altération du fond de fouille

A la fin de l'excavation, le géotechnicien vérifie la concordance du modèle géotechnique avec la nature et l'homogénéité du sol en fond de fouille.

En cas de niveaux d'assise différents, le géotechnicien s'assure que la règle des redans est respectée.

En cas d'apport de matériaux, leur classification et leur portance doivent être définies et contrôlées par le géotechnicien.

\section{10}

\section{Vis-à-vis des hypothèses hydrauliques}

Le géotechnicien vérifie la validité du modèle hydrogéologique, en particulier l'absence d'accumulation des eaux surfaciques au niveau du massif.

\section{2 .}

\section{Embase poids}

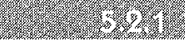 \\ Description}

Un massif de fondation d'éolienne a généralement une forme polygonale assimilable à un massif circulaire de même surface et de diamètre $\varnothing$. Sa base est généralement horizontale et se situe à une profondeur d'encastrement h par rapport au niveau de la surface.

Il s'agit de semelles considérées comme infiniment rigides.

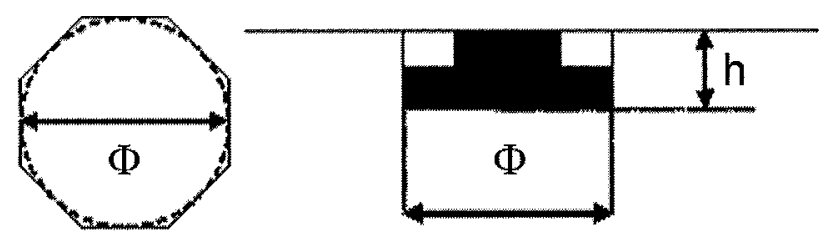

Fิ6. 8 Massif de fondation d'éoliennes.

Ce chapitre 5.2 s'applique d'une part aux embases poids réalisées directement sur le sol naturel mais également aux embases poids réalisées sur un sol substitué ou sur un sol amélioré (c dans la masse ) par des techniques spécifiques qui ne font pas l'objet de ces Recommandations (du type compactage dynamique, vibroflotation, injection solide).

\section{is:}

\section{Données géotechniques}

Conformément aux usages [Fascicule 62-Titre V (MELT 1993)], pour justifier la portance et pour calculer les tassements et la rotation d'un massif de fondation, il faut connaître le sol sur une hauteur théorique égale à $\mathrm{h}+8 \varnothing$, hauteur qu'on peut limiter dans le cas des massifs d'éoliennes aux valeurs décrites au $\$ 4.4 .3 .1$.

La portance est calculée à partir d'une pression limite équivalente ( $\left.p_{l e}{ }^{*}\right)$ ou d'une valeur pénétrométrique équivalente $\left(\left(\mathrm{q}_{\mathrm{ce}}\right)\right.$.

Ces valeurs de $p_{\text {le. }}$ et/ou de $q_{\mathrm{ce}^{\prime}}$ en fonction d'une gamme de diamètres $\Phi$ sont données dans le cadre d'une étude géotechnique.

\section{8}

\section{Justifications}

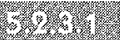

Portance

La portance se fait par application des règlements en vigueur (exemple développé ci-dessous : règles pressiométriques et pénétrométriques) à l'ELS et à l’ELU.

Dans tous les cas de charges ELS et ELU, on calcule $\sigma_{\max } \sigma_{\min }$ et $q_{\text {ref }}=\left(3 \sigma_{\max }+\sigma_{\text {min }}\right) / 4$ conformément au $\S 3.5 .2$.

On vérifie pour la contrainte maxi $q_{\text {ref }}$ que :

$$
\begin{aligned}
& q_{\text {ref }}<i_{\delta \beta} k_{p} p_{l e}{ }^{*} / \gamma_{\text {sol }}+q^{\prime} \\
& q_{\text {ref }}<i_{\delta \beta} k_{c} q_{\text {ce }} / \gamma_{\text {sol }}+q^{\prime}
\end{aligned}
$$

où $\gamma_{\text {sol }}$ est le coefficient partiel de sécurité des règlements en vigueur sous semelle.

Commentaire : $i_{\delta \beta}$ calculé selon la norme en vigueur en fonction de l'inclinaison de la charge appliquée et de la proximité de la pente d'un talus.

Commentaire : En cas d'essais pressiométriques, on s'assure que la pression maximale appliquée sur le sol n'excède pas la pression de fluage $p_{f}$

\section{9.}

\section{Tassement}

\section{Tassement global}

Dans le domaine de déformations statiques comprises entre $10^{-3}$ et $10^{-2}$, sous une charge apportant une contrainte $q$, on peut calculer le tassement $w$, et donc déterminer une raideur $\mathrm{K}_{\mathrm{vs}}=\mathrm{q} / \mathrm{w}$ statique à CT et à LT.

On rappelle que ces valeurs de $\mathrm{K}_{\mathrm{vS}}$ à CT et à LT sont données dans le cadre d'une étude géotechnique.
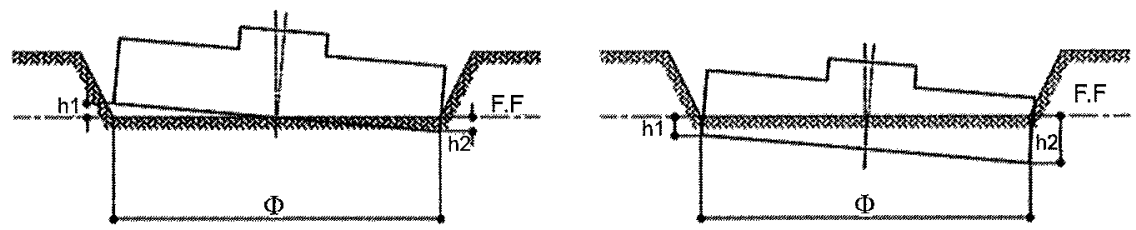


\section{1.}

\section{Rotation}

La rotation est définie à partir des grandeurs de la figure 9 par la formule suivante :

$$
\varphi=\left(h_{2}-h_{1}\right) / \Phi
$$

La raideur en rotation est définie comme $K_{\varphi}=M / \varphi$

Cas particulier. du sol entièrement comprimé

Le tableau suivant donne les expressions littérales permettant de déterminer les valeurs des coefficients de raideur des ressorts pour des massifs circulaires rigides de rayon $r$ dans le cadre d'un milieu parfaitement élastique homogène semi-indéfini et isotrope.

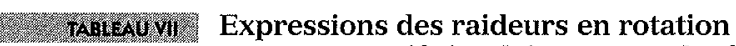
pour un massif circulaire non soulevé.

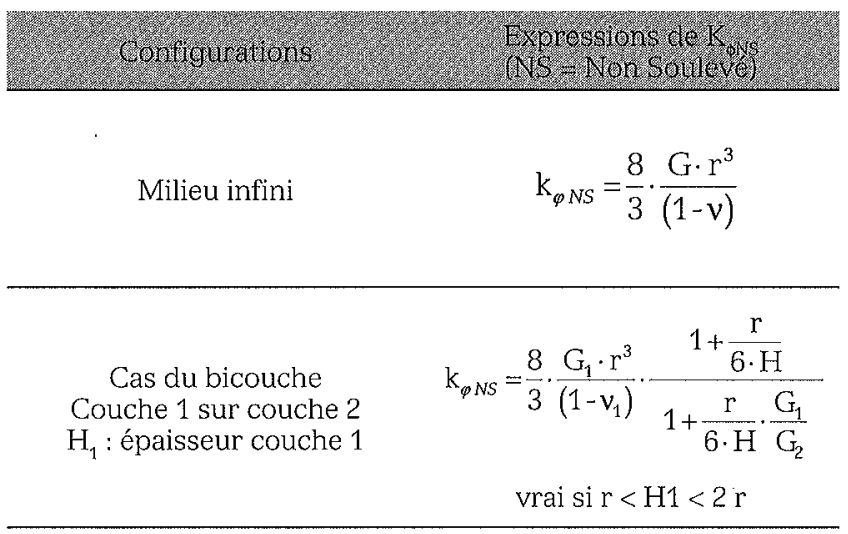

$$
\operatorname{avec} \mathrm{G}=\mathrm{E} /[2(1+v)]
$$

Commentaire : Ces expressions, rapportées à l'axe principal d'inertie, ne sont valables que dans la mesure où le sol reste comprimé sous la totalité du massif circulaire de rayon $r=\Phi / 2$.

Il est également rappelé que, sous ELS quasi permanent (obtenu à partir du cas de charge $\mathrm{DLC}_{\mathrm{QP}}$ ), le sol sous la semelle doit toujours être entièrement comprimé.

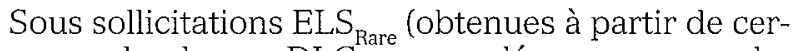
tains cas de charge DLC ${ }_{\text {Rare }}$ rappelés au paragraphe 3.4 .1 ( s situations conceptuelles $n^{\circ} 1$ et $\left.n^{\circ} 2\right)$ ), le sol peut ne pas être entièrement comprimé ; il faut alors pondérer $K_{\varphi}$ d'un coefficient $\beta_{1}$ (cf. $§$ 3.5.5.1 et figure 3) qui dépend du pourcentage de sol entièrement comprimé sous la semelle.

\section{6.}

\section{Vérification au glissement}

On se référera au paragraphe 3.5 .3 des présentes Recommandations.

\section{3}

\section{Embase poids sur sols renforcés par colonnes ballastées}

Ce chapitre s'applique exclusivement au renforcement de sol par la technique des colonnes ballastées sous les massifs de fondation d'éoliennes.
Il s'agit de semelles considérées comme infiniment rigides.

Les colonnes ballastées sont réalisées et contrôlées conformément aux « Recommandations colonnes ballastées du CFMS (2011) ) et de la norme NF EN 14731 (Amélioration des massifs de sols par vibration). Les présentes recommandations de ce document viennent en supplément de ces documents de références, pour tenir compte du caractère spécifique des fondations d'éoliennes. En cas de divergence, la condition ou la méthode la plus défavorable doit être utilisée.

\section{6.}

\section{Description}

Ce type de renforcement de sol consiste à mettre en place un groupe de colonnes verticales, constituées de matériaux granulaires, sans cohésion, mis en place par refoulement dans le sol et compactés par passes successives.

Ces colonnes traversent les sols compressibles de manière à améliorer et à homogénéiser les conditions de sol sous la fondation.

En complément du paragraphe 4.2 des « Recommandations colonnes ballastées du CFMS (2011) ), pour le cas particulier des éoliennes, le transfert des charges (en particulier de cisaillement) doit se faire par l'intermédiaire d'une couche de répartition en sousface du massif de fondation.

Commentaire : Si les colonnes ballastées sont réalisées depuis le fond de fouille sur une plate-forme de travail en matériau naturel granulaire, cette plateforme peut faire partie intégrante de cette couche de répartition. Si les colonnes ballastées sont réalisées depuis le terrain naturel avant excavation, il convient de mettre en place un matelas de répartition entre la tête des colonnes et la sous-face de la fondation.

Le traitement d'un sol par colonnes ballastées conjugue les actions suivantes, dont une seule ou plusieurs peuvent être recherchées :

- amélioration de la portance, réduction des tassements :

- augmentation des caractéristiques équivalentes du massif de sol traité (la résistance au cisaillement horizontal, l'angle de frottement interne et les paramètres de déformation).

Une colonne ballastée est un procédé de renforcement de sol : ce n'est ni un élément de fondation, ni une fondation profonde, et la fondation d'un ouvrage reposant sur un sol traité par colonnes ballastées est toujours de type superficiel.

Les paramètres de dimensionnement du renforcement de sol sont alors les suivants :

- la profondeur L des colonnes ballastées ;

- la surface de la section droite de ces inclusions et/ou leur diamètre équivalent $\Phi_{1}$ dans chacune des couches traversées;

- la contrainte admissible dans la colonne ballastée (dépendante des caractéristiques mécaniques du sol encaissant), ses caractéristiques de déformation (module de déformation, coefficient de Poisson...) ;

- le nombre de colonnes;

- le maillage des colonnes ou alternativement le taux d'incorporation $\beta$ du renforcement représentant le rapport de l'aire couverte par les têtes de colonnes et l'aire totale de la surface traitée ; 
- les caractéristiques du matelas de répartition;

- l'épaisseur $\mathrm{H}_{\text {mat' }}$

- les caractéristiques intrinsèques : $c^{\prime}$ et $\varphi^{\prime}$.

- les modules de déformation $E$ et de cisaillement $G$,

- sa compacité.

\section{res}

\section{Données géotechniques}

On se référera au chapitre 4 ( Géotechnique et paramètres de dimensionnement » des présentes Recommandations.

Pour justifier la portance et pour calculer les tassements et la rotation de ce massif sur sol renforcé, on rappelle que les données géotechniques doivent combiner :

- les exigences du chapitre 5.1.2 sur les embases poids, d'une part.

- les exigences propres aux calculs de la portance des colonnes ballastées d'autre part, à savoir une connaissance du sol sur une hauteur égale à $L+\max (5 \mathrm{~m}$; $\left.7 \Phi_{1}\right)$.

Les études géotechniques successives doivent permettre de préciser une coupe type, avec par couche sensiblement homogène l'ensemble des paramètres de sol listés aux paragraphes 4.6.1 et 4.6.2.

Commentaire : On rappelle que les valeurs de $p_{1}^{*}$ ou de $q_{c}$ pour calculer l'étreinte latérale de la colonne ballastée doivent être données dans le cadre d'une étude géotechnique.

\section{1.}

\section{Fonctionnement des colonnes ballastées}

\section{3.}

\section{Principes généraux}

On admet les hypothèses suivantes :

- les charges apportées par la fondation sont réparties entre le sol et les colonnes ballastées en fonction des raideurs verticales et de leur taux d'incorporation, par diffusion des contraintes à travers la couche de répartition;

- le chargement des colonnes ballastées, et par conséquent la répartition des charges, est limité par l'étreinte latérale mobilisable dans le terrain environnant (étreinte latérale fonction de la pression limite, ou de la résistance de pointe au pénétromètre statique, cf. chapitre 5.4 .1 des « Recommandations colonnes ballastées du CFMS (2011) )).

\section{6.}

\section{Domaines d'application}

Pour les projets d'éoliennes, on impose que les colonnes ballastées ne doivent pas être utilisées dans les sols compressibles qui ne permettent pas de garantir un confinement latéral suffisant.

On attire 1'attention sur les difficultés de justification des contraintes admissibles dans le sol et dans les colonnes (essentiellement sous les sollicitations de type ELU) sauf à augmenter le diamètre du massif de façon à réduire sensiblement les contraintes appliquées sous le massif.
Commentaire : Dans les sols compressibles, il est en effet en général difficile de justifier un massif sur colonnes ballastées avec un taux de travail sous le massif supérieur à $250 \mathrm{kPa}(2,5$ bars $)$ à l'ELS et supérieur à $350 \mathrm{kPa}(3,5$ bars $)$ à l'ELU.

\section{3.}

\section{Objectifs du renforcement de sol}

L'objectif du renforcement de sol est de donner au sol renforcé des caractéristiques mécaniques suffisantes pour permettre la construction d'une éolienne sur un massif poids classique. La fondation doit pouvoir se comporter de la même manière que sur un sol homogène.

Les paramètres à déterminer nécessaires au dimensionnement du massif sont ainsi les suivants :

- ceux permettant le calcul de la capacité portante ELS/ ELU du sol renforcé ;

- les coefficients de réaction $\mathrm{K}_{v}$ et $\mathrm{K}_{\mathrm{h}}$ de la fondation ou modules de déformation équivalent $\mathrm{E}_{\text {eq }}$ du sol renforcé à CT et à LT ;

- ceux permettant la vérification du glissement, en particulier l'angle de frottement $\varphi$ du sol sous la fondation (qui correspond à l'angle de frottement de la fondation sur le matelas de répartition) ;

- le coefficient de Poisson équivalent $v_{\text {eq }}$ du sol renforcé ;

- le module de cisaillement dynamique équivalent $\mathrm{G}_{\mathrm{dyneq}}$ du sol renforcé dans le domaine de déformation $10^{-3}$ a $10^{-4}$ et le coefficient de raideur dynamique en rotation $\mathrm{K}_{\text {pdyn }}$ de la fondation sur sol renforcé.

\subsection{5.}

\section{Justifications}

\section{3.}

\section{Vérification de la portance}

On doit vérifier, lors des calculs de dimensionnement, que pour tous les cas de charge ELS et ELU la répartition des contraintes entre le sol et les colonnes ballastées n'entraîne pas de dépassement des valeurs limites $q_{S}$ sous la semelle et $q_{a} / q_{a E L U}$ dans les colonnes (cf. définition de $q_{a}$ et $q_{a E L u}$ dans les paragraphes 5.4.4 et 5.4.5 des « Recommandations colonnes ballastées du CFMS ») et cela pour toutes les colonnes ballastées.

Dans tous les cas ELS ou ELU, on calcule $\mathrm{S}_{\text {comp }^{\prime}} \sigma_{\max }$ $\sigma_{\text {min }}$ et $q_{\text {ref }}=\left(3 \sigma_{\max }+\sigma_{\text {min }}\right) / 4$ conformément au $\$ 3.5 .2$.

Commentaire : Pour qu'une colonne soit prise en compte dans le calcul de portance globale, il faut vérifier qu'elle se situe dans la partie fictive du sol entièrement comprimée telle que proposée au \$3.5.2 et dessinée dans l'annexe $B$.

Pour tenir compte du caractère spécifique des fondations d'éoliennes, et dans le cadre des méthodes pressiométriques et pénétrométriques, on doit vérifier dans tous les cas de charges les critères de portance globale et de portance locale décrits aux paragraphes suivants. 


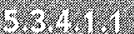

Critère de portance globale

Le critère suivant de portance globale est vérifié pour tous les cas de charges ELS et ELU avec :

$$
\begin{gathered}
\mathrm{q}_{\text {solELS }}>\left(\mathrm{q}_{\text {refELS }} \mathrm{S}_{\text {comp }}-\mathrm{n} \mathrm{Q}_{\text {colELS }}\right) /\left(\mathrm{S}_{\text {comp }}-\mathrm{n} \mathrm{A}_{\mathrm{p}}\right) \\
\mathrm{q}_{\text {solELU }}>\left(\mathrm{q}_{\text {refELU }} \mathrm{S}_{\text {comp }}-\mathrm{n} \mathrm{Q}_{\text {colELU }}\right) /\left(\mathrm{S}_{\text {comp }}-\mathrm{n} \mathrm{A}_{\mathrm{p}}\right)
\end{gathered}
$$

$\mathrm{n}=$ nombre de colonnes sous la surface de référence $\mathrm{S}_{\text {ref }}$ dessinée en annexe $\mathrm{B}$

$$
\begin{aligned}
& Q_{\text {colELS }}=A_{p} q_{a E L S} \\
& Q_{\text {colELU }}=A_{p} q_{a E L U}
\end{aligned}
$$

avec les méthodes pressiométriques ou pénétrométriques, on utilise respectivement:

$$
\begin{aligned}
& \mathrm{q}_{\text {solELU }}=\mathrm{k}_{\mathrm{p}} \mathrm{p}_{\mathrm{le}} / \gamma_{\text {solELU }}+\mathrm{q}_{\mathrm{o}}^{\prime} \text { ou } \\
& \mathrm{q}_{\text {solELU }}=\mathrm{k}_{\mathrm{c}} \mathrm{q}_{\text {ce }} / \gamma_{\text {solELU }}+\mathrm{q}_{\mathrm{O}} \mathrm{O} \\
& \mathrm{q}_{\text {solELS }}=\mathrm{k}_{\mathrm{p}} \mathrm{p}_{\mathrm{le}} / \gamma_{\text {solELS }}+\mathrm{q}_{\mathrm{o}}^{\prime} \text { ou } \\
& \mathrm{q}_{\text {solELS }}=\mathrm{k}_{\mathrm{c}} \mathrm{q}_{\mathrm{c}} / \gamma_{\text {solELS }}+\mathrm{q}^{\prime} \mathrm{o}
\end{aligned}
$$

avec $\gamma_{\text {solELS }}=3$ et $\gamma_{\text {solELU }}=2$

\section{4.}

Critère de portance locale

On doit vérifier maille par maille pour tous les cas de charges (ELS et ELU) :

- les critères suivants de portance du sol :

$$
\begin{gathered}
q_{\text {solELS }}>\left(q_{\text {refELS }} S_{\text {maille }}-Q_{\text {colELS }}\right) /\left(S_{\text {maille }}-A_{p}\right) \\
q_{\text {solELU }}>\left(q_{\text {refELU }} S_{\text {maille }}-Q_{\text {colELU }}\right) /\left(S_{\text {maille }}-A_{p}\right) \\
Q_{\text {colELS }}=A_{p} q_{a E L S} \\
Q_{\text {colELU }}=A_{p} q_{\text {aELU }}
\end{gathered}
$$

avec les méthodes pressiométriques ou pénétrométriques, on utilise aussi respectivement les équations suivantes :

$$
\begin{gathered}
q_{\text {solELU }}=\mathrm{k}_{\mathrm{p}} \cdot \mathrm{p}_{\mathrm{le}} / \gamma_{\text {solELU }}+\mathrm{q}_{\circ}^{\prime} \text { ou } \mathrm{q}_{\text {solELU }}=\mathrm{k}_{\mathrm{c}} \cdot \mathrm{q}_{\mathrm{ce}} / \gamma_{\text {solELU }}+\mathrm{q}_{\mathrm{o}}^{\prime} \\
\mathrm{qS}_{\text {olELS }}=\mathrm{k}_{\mathrm{p}} \cdot \mathrm{p}_{\mathrm{le}} / \gamma_{\text {solELS }}+\mathrm{q}_{\mathrm{o}}^{\prime} \text { ou } \mathrm{q}_{\text {solELS }}=\mathrm{k}_{\mathrm{c}} \cdot \mathrm{q}_{\mathrm{ce}} / \gamma_{\text {solELS }}+\mathrm{q}_{\mathrm{o}}^{\prime} \\
\text { avec } \gamma_{\text {solELS }}=3 \text { et } \gamma_{\text {solELU }}=2
\end{gathered}
$$

- les critères suivants à l'ELS pour les tassements afin qu'ils restent élastiques :

$$
\mathrm{q}_{\text {app }}^{\prime}<\mathrm{k}_{\mathrm{p}} \mathrm{p}_{\mathrm{le}} / \gamma+\mathrm{q}_{\mathrm{o}}^{\prime} \text { ou bien } \mathrm{q}_{\mathrm{app}}^{\prime}<\mathrm{k}_{\mathrm{c}} \mathrm{q}_{\mathrm{ce}} / \gamma+\mathrm{q}_{\mathrm{o}}^{\prime} \text { (38) }
$$
où $\mathrm{q}_{\text {app }}^{\prime}$ est la contrainte reprise moyenne par le sol sur la maille

- les critères suivants pour les contraintes dans les colonnes :

$\mathrm{q}_{\mathrm{col}}<\mathrm{q}_{\mathrm{aELS}}$ à l'ELS limitée à min $\left(\mathrm{q}_{\mathrm{re}} ; \mathrm{q}_{\mathrm{rp}} ; 1,6 \mathrm{MPa}\right) / 2$;

$q_{\text {col }}<q_{a E L U}$ à l'ELU limitée à $\min \left(q_{r e} ; q_{r p} ; 1,6 \mathrm{MPa}\right) / 1,5$;

$\mathrm{q}_{\mathrm{aELS}}$ : contrainte maximale admissible dans la colonne à I'ELS ;

$\mathrm{q}_{\mathrm{aELU}}$ : contrainte maximale admissible dans la colonne à l'ELU ;

$\mathrm{q}_{\mathrm{re}}$ et $\mathrm{q}_{\mathrm{rp}}$ : cf. définitions $\$ 5.4$ dans les « Recommandations colonnes ballastées ) du CFMS, 2011.

\section{3 in}

\section{Vérification au glissement}

Les colonnes ballastées permettent d'augmenter les caractéristiques équivalentes du massif de sol traité : résistance au cisaillement horizontal, angle de frottement interne et éventuellement paramètres de déformation.

Les efforts de cisaillement au niveau de la sousface de la semelle sont répartis à travers le matelas de répartition en fonction du frottement sous la semelle, donc au prorata de la répartition des contraintes verticales en compression :

- respectivement $\sigma_{\mathrm{col}}$ dans la colonne ballastée;

- et $\sigma_{\mathrm{s}}$ hors emprise de cette colonne ballastée.

Ils ne s'appliquent donc que sur les sols ou sur les colonnes travaillant en compression, en particulier en cas de moments de renversement s'appliquant sur la semelle.

Dans le cas d'une semelle soumise à un torseur $(Q$, $\mathrm{M}, \mathrm{H}_{\mathrm{ELU}}$ ), seules les colonnes sollicitées en compression sont prises en compte dans la vérification.

En fonction de la part de la charge totale reprise respectivement par le sol et les colonnes ballastées, on peut déterminer à partir des angles de frottement internes la résistance au cisaillement équivalente pour l'ensemble sol/colonne ballastée.

avec

$$
\tan \varphi_{\mathrm{eq}}=\mathrm{m}^{\prime} \tan \varphi_{\mathrm{c}}+\left(1-\mathrm{m}^{\prime}\right) \tan \varphi_{\mathrm{s}}
$$

$$
m^{\prime}=(n-1) / n
$$

$$
\mathrm{n}=\text { facteur } \mathrm{d}^{\prime} \text { amélioration }=\sigma_{\text {appl }} / \sigma \mathrm{sol}
$$

On se référera alors au commentaire du paragraphe 3.5.3 en remplaçant $\varphi^{\prime} \operatorname{par} \varphi_{\text {eq }}$

\section{5}

\section{Calcul des déformations}

Le tassement et la rotation de la fondation sont calculés à partir des caractéristiques équivalentes du sol renforcé pour les charges de courte et de longue durée d’application selon les principes du § 5.2.3.

\section{6.}

\section{Comportement intrinsèque des colonnes ballastées}

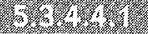

Contraintes maximales admissibles dans les colonnes

Le calcul de la contrainte maximale admissible consiste d'abord à déterminer la contrainte verticale de rupture $\mathrm{q}_{\mathrm{r}}$ d'une colonne isolée à partir des caractéristiques du matériau des colonnes et celles du sol encaissant, et cela selon les schémas de rupture possibles suivants :

- rupture par expansion latérale (critère souvent dimensionnant) ;

- rupture par poinçonnement (colonnes flottantes).

\section{(6)}

Module de déformation statique à 10-2 de la colonne ballastée

Ce module vaut au maximum 10 fois le module du sol encaissant. Selon le paragraphe 5.3 des « Recommandations colonnes ballastées ») du CFMS, 2011, il peut être pris égal à $\mathrm{E}=60 \mathrm{MPa}$ si les colonnes respectent les critères de compacité recherchés :

$$
\begin{aligned}
& \mathrm{q}_{\mathrm{cm}}>10 \mathrm{MPa} ; \\
& \mathrm{p}_{\mathrm{l}}>1,2 \mathrm{MPa} ; \\
& \mathrm{q}_{\mathrm{d}}>10 \mathrm{MPa} .
\end{aligned}
$$




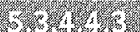

Sollicitations « dynamiques » à $10^{-4}$ dans la colonne ballastée

Si les colonnes respectent les critères minimum de compacité précédents, le module de cisaillement à $10^{-4}$ dans les colonnes ballastées peut être pris égal à :

$$
\mathrm{G}_{\text {coldyn }}=0,55 \mathrm{G}_{\max }=55 \mathrm{MPa}
$$

Commentaire : Cette valeur est obtenue en utilisant les corrélations suivantes: $G_{\max }=7 E_{m} ; E_{m}=15 \mathrm{MPa}$; $G_{\text {coldyn }} / G_{\max }=0,55$ à $10^{-4}$.

\section{$3 \sin ^{2}$}

\section{Calcul des caractéristiques (c dynamiques ) équivalentes du sol renforcé}

Pour les plages de déformations de $10^{-3}$ à $10^{-4}$, on fait l'hypothèse simplificatrice que les déformations de cisaillement du sol et des colonnes ballastées sont égales et que le module de cisaillement équivalent du système sol-colonne s'écrit (cf. § 4.6.2 et 5.3.4.4.3) :

$$
\begin{gathered}
G_{\text {eq. }}=\beta \cdot G_{\text {coldyn }}+(1-\beta) \cdot G_{\text {soldyn }} \\
\beta: \text { taux de substitution }=A_{\text {col }} / S_{\text {maille }} \\
A_{\text {col }} \text { : aire de la colonne ballastee } \\
S_{\text {maille }} \text { : surface d'une maille }
\end{gathered}
$$

Cette valeur de $\mathrm{G}_{\text {eq }}$ à $10^{-4}$ permet de calculer le critère de raideur en rotation $K_{\text {p }}$ par la formule du $\S 4$ 4.3.2.2 et d'appliquer le critère du $\S 3.5 .5$.

\section{a.s.}

\section{Dispositions constructives}

Les dispositions des règlements en vigueur ( $(\mathrm{Re}$ commandations colonnes ballastées », CFMS, 2011) et celles du paragraphe 5.1.2 s'appliquent et sont complétées ci-après.

\section{8}

\section{Colonnes de confinement}

La charge mobilisable dans les colonnes ballastées est limitée par l'étreinte latérale mobilisable dans le terrain environnant (fonction de la pression limite, ou de la résistance de pointe au pénétromètre statique, cf. chapitre 5.4 .12 des " Recommandations colonnes ballastées, CFMS, 2011 ).

Les massifs de fondation d'éolienne induisent spécifiquement des contraintes importantes en bord de fondation.

Si les méthodes de calcul utilisées pour le dimensionnement des colonnes ballastées considèrent un parfait confinement des colonnes et un maillage infini, il est nécessaire de renforcer le nombre de colonnes ballastées en périphérie sous le massif de fondation ou de disposer une rangée de colonnes périphériques pour assurer ce confinement.

On doit de plus prévoir des colonnes de confinement à l'extérieur de la semelle dans les cas suivants :

- dans le cas où le dimensionnement de la fondation tient compte d'une amélioration des caractéristiques du sol entre les colonnes (en particulier de l'étreinte latérale) due à leur mode d'exécution (serrage du terrain). Dans ce cas, des essais de réception entre colonnes (CPT, PMT...) devront être réalisés pour confirmer l'amélioration obtenue ;
- dans le cas de sol liquéfiable où la réalisation des colonnes ballastées a un rôle de traitement anti-liquéfaction). Le traitement doit alors être étendu sur une largeur de débord égale à la moitié de la profondeur de la base de la couche sensible à la liquéfaction.

\section{b.}

\section{Couche de répartition}

En complément du paragraphe 4.2 des « Recommandations colonnes ballastées du CFMS » et pour le cas particulier des éoliennes, le transfert des charges (en particulier de cisaillement) doit se faire par l'intermédiaire d'une couche de répartition en sous-face du massif de fondation.

Elle a pour but d'éviter le remaniement et d'assurer un contact homogène entre la semelle et le sol.

Commentaire : Si les colonnes ballastées sont réalisées depuis le fond de fouille sur une plate-forme de travail en matériau naturel granulaire, cette plateforme peut faire partie intégrante du matelas de répartition. Si les colonnes ballastées sont réalisées depuis le terrain naturel avant excavation, il convient de mettre en place un matelas de répartition entre la tête des colonnes et la sous-face de la fondation.

La couche de répartition de hauteur $\mathrm{H}_{\text {mat }}$ doit être mise en œuvre en fonction des critères de type “ couche de forme ) ou ( routiers » (LCPC/SETRA 2000a et b), qu'elle soit constituée de matériaux frottants (graves naturelles) et/ou renforcée de liants (ciments, chaux, etc.).

Elle est caractérisée par des essais in situ de type ( routiers ) ou de type (couche de forme » (essais à la plaque...), ou par des essais géotechniques plus classiques (type essais pressiométriques ou pénétrométriques), ou par des essais de laboratoire (indice CBR ou IPI, mesures de la cohésion, de l'angle de frottement et de la teneur en eau, etc.).

Ces essais ont pour but de pouvoir vérifier la compacité en place des matériaux et de déterminer sa loi de comportement par l'estimation ses différents modules de déformations usuels (module pressiométrique si possible, module d'Young E ou module œdométrique $M$ ) et de cisaillement $\left(\mathrm{c}^{\prime}, \varphi^{\prime}\right)$ et de pouvoir calculer le tassement et la résistance ultime à la compression du matériau de cette couche et sa résistance au cisaillement.

Ses caractéristiques géomécaniques, par exemple le module EV2, et l'épaisseur de ce matelas, sont fonction de l'étude de dimensionnement du système de fondation.

Généralement cette couche de répartition est constituée d'au moins $40 \mathrm{~cm}$ de matériaux :

- de remblai granulaire en grave naturelle ;

- par exemple de classe D1, D2 ou D3 ou R suivant LCPC/SETRA (2000a et b) (ou NFP 11-300),

- compactée à $95 \%$ de l'Optimum Proctor Modifié (OPM),

- ce qui lui conférera un module de déformation (équivalent à un module EV2) de l'ordre de $50 \mathrm{MPa}$, un rapport EV2/EV1 $<2,1$ et un angle de frottement de $40^{\circ}$ pour les matériaux concassés et $38^{\circ}$ pour les matériaux roulés,

- de sols traités au liant dont les caractéristiques usuelles de cohésion et d'angle de frottement à prendre en compte pour les calculs sont $c^{\prime}=50 \mathrm{kPa}$ et $\varphi^{\prime}=25^{\circ}$. 
La couche de répartition sera mise en ouvre selon les règles de l'art avec les contrôles usuels de réception appliqués aux couches de formes sous dallage.

Afin de répartir le mieux possible la concentration des efforts en périphérie de massif, un débord de matelas est nécessaire sur une largeur correspondant au minimum à $\max \left(\mathrm{H}_{\mathrm{mat}} / 2 ; 0,5 \mathrm{~m}\right)$ au-delà du bord du massif et de la dernière rangée de colonnes, correspondant à la largeur minimale pour assurer un compactage satisfaisant.

On prendra des dispositions constructives nécessaires pour que le matelas de répartition ne soit pas contaminé par le sol support (géotextile, couche anti-contaminante...).

\section{8.}

\section{Vérifications et contrôles}

Ils seront conformes à ceux du chapitre 6 des « Recommandations colonnes ballastées ), CFMS, 2011 et complétés par les suivants.

\section{8.}

\section{Portance et altération du toit de l'excavation}

On se référera au paragraphe 5.1.3.

\section{S.8.}

\section{Couche de répartition}

\section{2.}

Épaisseur

On vérifie l'épaisseur du matelas par comparaison de relevés topographiques à raison de 3 points par éolienne.

\section{1.29}

Qualité

On recommande les essais de type « couche de forme m, essais de plaque, CBR ou IPI, ainsi que des mesures de $\varphi^{\prime}$, et de granulométrie.

Commentaire : En cas d'épaisseur de remblai supérieure à $1 \mathrm{~m}$, on peut utiliser le pressiomètre ou le pénétromètre statique.

Les densités de ces différents types d'essais peuvent être les suivantes:

- essais de portance (plaque, $\mathrm{q}_{\mathrm{c}^{\prime}} \mathrm{p}_{1}$ ou CBR, au choix) ; un minimum de 3 par massif et 3 par chantier, - essais d'identification (granulométrie) et/ou de caractérisation $\left(c^{\prime}, \varphi^{\prime}\right)$;

un minimum de 1 par chantier.

\section{4}

\section{Embase poids sur sols renforcés par inclusions rigides}

Pour tenir compte du caractère spécifique des fondations d'éoliennes, les recommandations de ce paragraphe 5.4 viennent en complément des recommandations du Projet national ASIRI (Amélioration des Sols par Inclusions RIgides) et des cahiers des charges de l'entrepreneur.

\section{1.}

\section{Description}

Un massif de fondation d'éolienne a généralement une forme polygonale assimilable à un massif circulaire de même surface et de diamètre $\Phi$. Sa base est généralement horizontale et se situe à une profondeur h du niveau de la surface. Il s'agit de semelles considérées comme infiniment rigides.

Le massif de fondation repose sur un sol amélioré par un groupe de $n$ inclusion rigides (IR) de diamètre équivalent $\Phi_{1}$ et de longueur L.
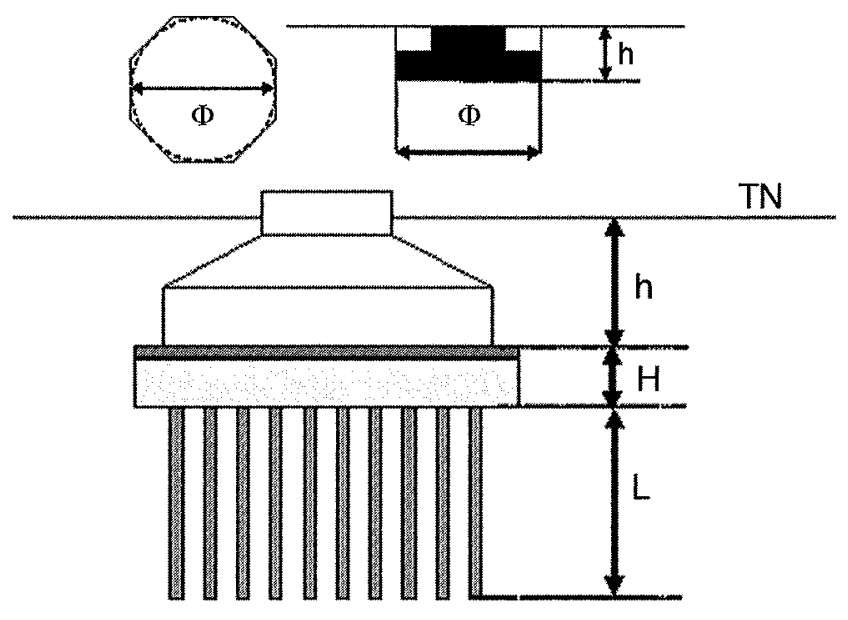

1E. 10. Schéma du renforcement.

Ce paragraphe 5.4 s'applique exclusivement au renforcement de sol par la technique des inclusions rigides verticales sous les massifs de fondation d"éolienne.

Ce type de renforcement de sol consiste à mettre en place un groupe d'inclusions rigides verticales traversant les sols compressibles de manière à améliorer et à homogénéiser les conditions de sol sous la fondation. en créant un matériau composite :

- ces inclusions peuvent être mises en œuvre suivant des méthodes très variées (foration, percussion, vibration, refoulement...);

- ces inclusions sont généralement de type Inclusion en béton ou mortier ou de type Inclusions métalliques.

Le transfert de charge depuis le massif de fondation se fait alors nécessairement par l'intermédiaire d'un matelas de répartition intercalé entre l'arase inférieure de la fondation et la tête des inclusions. Dans le cas contraire, on appliquera les principes des fondations mixtes ou des ( fondations composites ) (cf. \$ 5.6).

Les paramètres de dimensionnement du renforcement de sol sont alors les suivants :

- la profondeur L des inclusions ;

- la surface de la section droite de ces inclusions et/ou leur diamètre équivalent $\Phi_{1}$;

- la résistance du matériau de cette inclusion, ses caractéristiques de déformation (module de déformation, coefficient de Poisson...) ;

- les critères de portance de ces inclusions vis-à-vis du sol ; 
- le nombre des inclusions ; le maillage des inclusions ou alternativement le taux d'incorporation $\alpha$ du renforcement représentant le rapport de l'aire couverte par les têtes d'inclusions et l'aire totale de la surface traitée ;

- les caractéristiques du matelas de répartition ;

- l'épaisseur $\mathrm{H}_{\text {mat' }}$

- les caractéristiques intrinsèques : $\mathrm{c}^{\prime}$ et $\varphi^{\prime}$,

- les modules de déformation $\mathrm{E}$ et de cisaillement $\mathrm{G}$

- sa compacité.

Ce système de renforcements de sols s'applique préférentiellement dans les domaines $n^{\circ} 1$ et $n^{\circ} 2$, en tenant compte du commentaire du paragraphe 5.1.

\section{8.}

\section{Données géotechniques}

Pour justifier la portance et pour calculer les tassements et la rotation de ce massif sur sol renforcé, on rappelle que les données géotechniques doivent combiner :

- les exigences du chapitre 5.1.2 sur les embases poids d'une part ;

- les exigences propres aux calculs de la portance des inclusions rigides d'autre part, à savoir une connaissance du sol sur une hauteur égale à $\mathrm{h}+\mathrm{H}+\mathrm{L}+\max (5 \mathrm{~m}$; $\left.7 \varnothing_{1} ; \varnothing / 2\right)$.

Les études géotechniques successives doivent permettre de préciser une coupe type, avec par couche sensiblement homogène l'ensemble des paramètres de sol listés au $\$ 4.6 .1$ et 4.6.2.

\section{3.}

\section{Fonctionnement des inclusions rigides}

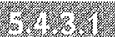

\section{Efforts verticaux de structure}

On admet les hypothèses suivantes:

- les charges des structures sont réparties entre le sol et les inclusions rigides :

- par diffusion des contraintes à travers la couche de répartition,

- par un transfert de charges « sol - inclusions » et ( inclusions - sol ), sous l'effet des déplacements relatifs des inclusions et du sol environnant en raison de leur différence de raccourcissement (comportement élastique),

- le chargement des inclusions rigides est limité par l'effet de leur tassement dans la couche d'assise en pied d'inclusions et par la pénétration de l'inclusion dans la couche de répartition (comportement élasto-plastique du contact ( inclusion-couche de répartition ») et « inclusion-ancrage $)$ ).

\section{6.}

\section{Efforts non verticaux de structure}

Les inclusions rigides ne sont pas faites pour reprendre directement les efforts horizontaux, les moments ou les efforts de soulèvement provenant de la structure.

Des dispositions spécifiques doivent être adoptées pour la reprise de ces efforts, et les méthodes classiques de dimensionnement de semelles superficielles (frottement, lestage...) sont applicables pour ces efforts.

Les inclusions, suivant leurs caractéristiques intrinsèques et les conditions de sol peuvent accepter de faibles valeurs d'efforts parasites (efforts horizontaux, cisaillement...) qu'il convient d'estimer et dont il convient de vérifier la compatibilité avec les critères de résistance des matériaux des inclusions (cf. § 5.4.4).

\section{(3)}

\section{Objectif du renforcement de sol}

L'objectif du renforcement de sol est de donner au sol renforcé des caractéristiques mécaniques suffisantes pour permettre la construction d'une éolienne sur un massif poids classique. La fondation doit pouvoir se comporter de la même manière que sur un sol homogène.

Les paramètres à déterminer nécessaires au dimensionnement du massif sont ainsi les suivants :

- ceux permettant le calcul de la capacité portante ELS/ ELU du sol renforcé ;

- les coefficients de réaction $K_{v}$ et $K_{h}$ de la fondation ou modules de déformation équivalent $\mathrm{E}_{\mathrm{eq}}$ du sol renforcé à CT et à LT ;

- ceux permettant la vérification du glissement, en particulier l'angle de frottement;

- égal à celui du matelas pour la vérification du glissement sur le plan horizontal de la sous-face de la semelle,

- égal à celui du sol pour la vérification du glissement sur le plan horizontal en sous-face du matelas,

- le coefficient de Poisson équivalent $v_{\mathrm{eq}}$ du sol renforcé ;

- le module de cisaillement dynamique équivalent $\mathrm{G}_{\text {dyne }}$ du sol renforcé dans le domaine de déformation $10^{-3}$ a $10^{-4}$ et le coefficient de raideur dynamique en rotation $\mathrm{K}_{\text {cdyn }}$ de la fondation sur sol renforcé.

La réalisation, le dimensionnement et le contrôle des inclusions rigides non définies dans le Fascicule 62-Titre $\mathrm{V}$ doivent impérativement être couverts par les recommandations ASIRI, ou par des cahiers des charges propres à la méthode de réalisation de l'entrepreneur et validés [conformément au DTU 13.2 \$ 1.11 $(+$ commentaire $)]$.

Une inclusion rigide est un procédé de renforcement de sol : ce n'est ni un élément de fondation, ni une fondation profonde.

Les méthodes réglementaires de dimensionnement utilisées pour les pieux de fondation ou pour les inclusions granulaires ne s'appliquent pas aux sols renforcés par Inclusions rigides.

\section{3.}

\section{Justification de l'inclusion}

\section{6.}

\section{Vérification de la portance du sol renforcé}

On doit vérifier, lors des calculs de dimensionnement, que la répartition des contraintes entre le sol et les inclusions n'entraîne pas de dépassement des valeurs limites d'interaction entre le sol et l'inclusion et cela pour toutes les IR, y compris entre le matelas et l'IR. 
On admet cependant, sous réserve de la vérification de la capacité portante globale et locale du système, que certaines de ces valeurs limites d'interaction soient partiellement saturées.

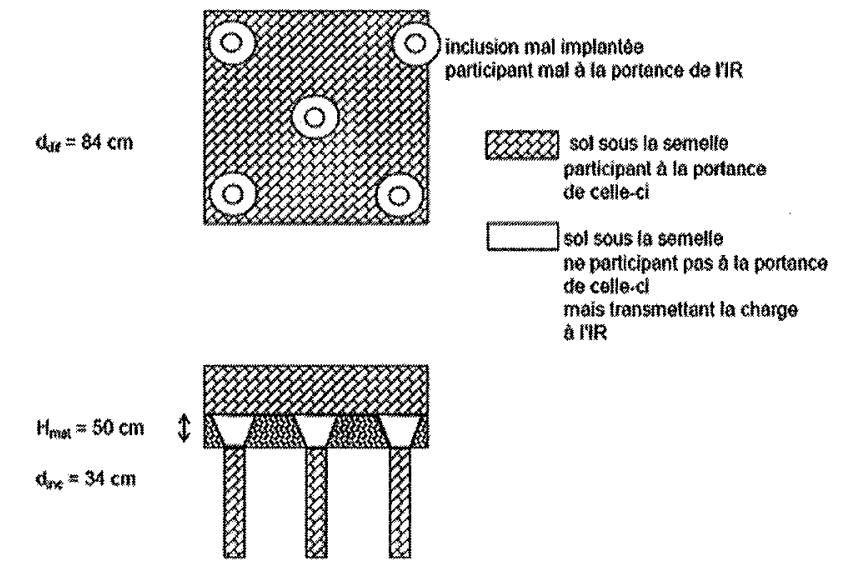

10.611 Exemple de répartition d'inclusions rigides sous une semelle.

Dans tous les cas ELS ou ELU, on calcule $S_{\text {comp' }} \sigma_{\text {max }}$ $\sigma_{\min }$ et $\mathrm{q}_{\mathrm{ref}}=\left(3 \sigma_{\max }+\sigma_{\min }\right) / 4$ conformément au paragraphe 3.5.2.

Commentaire : Pour qu'une colonne soit prise en compte dans le calcul de portance globale, il faut vérifier qu'elle se situe dans la partie fictive du sol entièrement comprimée telle que proposée au $\$ 3.5 .2$ et dessinée dans l'annexe $B$.

Pour tenir compte du caractère spécifique des fondations d'éoliennes, et dans le cadre des méthodes pressiométriques et pénétrométriques, on doit vérifier dans tous les cas de charges les critères de portance globale et de portance locale décrits aux paragraphes suivants.

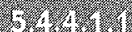

Critère de portance globale

Le critère suivant de portance globale est vérifié pour tous les cas de charges ELS et ELU avec :

$$
\begin{aligned}
& \mathrm{q}_{\text {solELS }}>\left(\mathrm{q}_{\text {refELS }} \mathrm{S}_{\text {ref }}-\mathrm{nQ}_{\text {colELS }}\right) /\left(\mathrm{S}_{\text {ref }}-\mathrm{n} A_{\mathrm{p}}\right) ; \\
& \mathrm{q}_{\text {solELU }}>\left(\mathrm{q}_{\text {refELU }} \mathrm{S}_{\text {ref }}-\mathrm{nQ}_{\text {colELU }}\right) /\left(\mathrm{S}_{\text {ref }}-\mathrm{n} A_{\mathrm{p}}\right) ;
\end{aligned}
$$

$\mathrm{n}=$ nombre d'inclusions sous la surface de référence $\mathrm{S}_{\text {ref }}$ dessinée en annexe $\mathrm{B}$;

$$
\begin{gathered}
Q_{\text {colELS }}=A_{p} \min \left[0,3 f_{c}{ }^{*} ;\left(R_{b} / \gamma_{b}+R_{s} / \gamma_{s}\right) / A_{p}\right] \\
Q_{\text {colELU }}=A_{p} \min \left[\alpha_{c c} f_{c}{ }_{c}^{*} / 1,5 ;\left(R_{b} / \gamma_{b}+R_{s} / \gamma_{s}\right) / A_{p}\right]
\end{gathered}
$$

$\alpha_{c c}=0,8$ pour les inclusions non armées, 1 sinon

- avec les méthodes pressiométriques ou pénétrométriques, on utilise respectivement

$$
\mathrm{q}_{\text {solELU }}=\mathrm{k}_{\mathrm{p}} \mathrm{p}_{\mathrm{le}} / \gamma_{\text {solELU }}+\mathrm{q}_{\mathrm{o}}^{\prime} \text { ou } \mathrm{q}_{\text {solELU }}=\mathrm{k}_{\mathrm{c}} \mathrm{q}_{\mathrm{ce}} / \gamma_{\text {solELU }}+\mathrm{q}_{\mathrm{o}^{\prime}}^{\prime}
$$$$
q_{\text {solELS }}=k_{p} p_{l e} / \gamma_{\text {solELS }}+q_{o}^{\prime} \text { ou } q_{\text {solELS }}=k_{c} q_{c e} / \gamma_{\text {solELS }}+q_{o}^{\prime} \text {. }
$$

\begin{tabular}{|c|c|c|c|c|c|}
\hline \multirow[t]{2}{*}{ ELS QP } & IR refoulée & 2,00 & 2,00 & & 3,00 \\
\hline & IR forée & 2,80 & 2,00 & & 3,00 \\
\hline \multirow[t]{2}{*}{ ELS Rare } & IR refoulée & 1,57 & 1,57 & & 3,00 \\
\hline & IR forée & 2,20 & 1,57 & & 3,00 \\
\hline ELU & & 1,40 & 1,40 & 1,75 & 2,00 \\
\hline
\end{tabular}

Vis-à-vis du critère de portance globale, les coefficients retenus pour l'application des formules sont les suivants :
Westraxuvil Coefficients de sécurité proposés.

Compléments : Dans la limite de la vérification de la capacité portante globale du système avec l'application des coefficients de sécurité ci-dessus, il peut apparaitre lors des calculs de la répartition des contraintes entre le sol, le matelas et les inclusions que des valeurs limites de certains des éléments résistants (sol sous la semelle, matelas, frottement de l'inclusion et pointe de l'inclusion) soient atteintes sans être dépassées (coefficient partiel de sécurité de 1 en fonctionnement

\section{6.}

Critère de portance locale

On doit vérifier maille par maille pour tous les cas de charges (ELS et ELU) que le critère suivant de portance locale est vérifié avec :

$$
\begin{gathered}
\mathrm{q}_{\text {solELS }}>\left(\mathrm{q}_{\text {refELUU }} \mathrm{S}_{\text {maille }}-\mathrm{Q}_{\text {colELU }}\right) /\left(\mathrm{S}_{\text {maille }}-\mathrm{A}_{\mathrm{p}}\right) \\
\mathrm{q}_{\text {solELU }}>\left(\mathrm{q}_{\text {refELS }} \mathrm{S}_{\text {maille }}-\mathrm{Q}_{\text {coleLS }}\right) /\left(\mathrm{S}_{\text {maille }}-\mathrm{A}_{\mathrm{p}}\right) \\
\mathrm{q}_{\text {colELS }}=\mathrm{A}_{\mathrm{p}} \min \left[0,3 \mathrm{f}_{\mathrm{c}}^{*} ; \max \left(\mathrm{S}_{\mathrm{d}^{\prime}} \mathrm{A}_{\mathrm{p}}\right) / \mathrm{A}_{\mathrm{p}} \sigma_{\text {mat }}^{\prime} / \gamma_{\text {mat }} ;\right. \\
\left.\left(\mathrm{R}_{\mathrm{b}} / \gamma_{\mathrm{b}}+\mathrm{R}_{\mathrm{s}} / \gamma_{\mathrm{s}}\right) / \mathrm{A}_{\mathrm{p}}\right] \\
\mathrm{q}_{\text {colELU }}=\mathrm{A}_{\mathrm{p}} \min \left[\alpha_{\mathrm{cc}} \mathrm{f}_{\mathrm{c}}^{*} / 1,5 ; \max _{\mathrm{d}} / \mathrm{S}_{\mathrm{d}} ; \mathrm{A}_{\mathrm{p}}\right) / \mathrm{A}_{\mathrm{p}} \sigma_{\text {mat }}^{\prime} / \gamma_{\text {mat }} ; \\
\left.\left(\mathrm{R}_{\mathrm{b}} / \gamma_{\mathrm{b}}+\mathrm{R}_{\mathrm{s}} / \gamma_{\mathrm{s}}\right) / \mathrm{A}_{\mathrm{p}}\right]
\end{gathered}
$$

Commentaire : On rappelle que la portance de la $\mathrm{CO}^{-}$ lonne fictive $\left(Q_{c o l}=q_{c o l} . S_{c o l}\right)$ est égale au minimum de la portance de l'inclusion en termes de résistance de matériau, de la portance de l'inclusion vis-à-vis du sol évaluée sous le point neutre et de la portance de la colonne de remblais au-dessus de l'inclusion en termes de poinçonnement :

$\sigma_{\text {mat }}^{\prime}$ et $\gamma_{\text {mat }}$ définis au paragraphe 5.4.5,

avec les méthodes pressiométriques ou pénétrométriques, on utilise respectivement

$$
\begin{aligned}
& \mathrm{q}_{\text {solELU }}=\mathrm{k}_{\mathrm{p}} \mathrm{p}_{\text {le }} / \gamma_{\text {solELU }}+\mathrm{q}_{\mathrm{o}}^{\prime} \text { ou } \mathrm{q}_{\text {solELU }}=\mathrm{k}_{\mathrm{c}} \mathrm{q}_{\mathrm{ce}} / \gamma_{\text {solELU }}+\mathrm{q}_{\mathrm{o}}^{\prime} \\
& \mathrm{q}_{\text {solELS }}=\mathrm{k}_{\mathrm{p}} \mathrm{p}_{\mathrm{le}} / \gamma_{\text {solELS }}+\mathrm{q}_{\mathrm{o}}^{\prime} \text { ou } \mathrm{q}_{\text {solELS }}=\mathrm{k}_{\mathrm{c}} \mathrm{q}_{\mathrm{ce}} / \gamma_{\text {solELS }}+\mathrm{q}_{\mathrm{o}}^{\prime}
\end{aligned}
$$

Les coefficients de sécurité sont ceux du tableau VIII

\section{6) 1 .}

\section{Calcul des déformations}

Le tassement et la rotation de la fondation sont calculés à partir des caractéristiques équivalentes du sol renforcé pour les charges de courte et de longue durée d'application selon les principes du paragraphe 5.2.3.

Il convient de prendre en compte les variations significatives de ces caractéristiques équivalentes en fonction de la position de la maille, des différences de conditions géotechniques et des charges appliquées.

\section{1.}

\section{Vérification en compression}

Le comportement intrinsèque des inclusions rigides est caractérisé par : 
- le module d’Young $E_{\mathrm{Y}}$ du matériau de l'élément rigide pour des charges de longue et de courte durée d'application;

- la résistance à la compression $\mathrm{f}_{c j}$ du matériau de l'élément rigide, contrôlée par écrasement d'éprouvettes dans le cas d'inclusions en béton;

- la nuance, la qualité et la limite élastique $\mathrm{f}_{\mathrm{s}}$ du matériau dans le cas d'inclusions métalliques.

\section{5.}

Inclusions en béton, coulis ou mortier

- Définition de $f_{c d}$

En cas de mise en cuvre de béton ou de coulis, la valeur caractéristique de calcul $f_{c d}$ du matériau est définie selon la Norme d'application Nationale de l'Eurocode 7 (NFP 94262 Fondations profondes) ou les cahiers des charges particuliers :

$$
f_{c d}=\operatorname{Min}\left(\alpha_{c c} k_{3} \frac{f_{c k}^{*}}{\gamma_{C}} ; \alpha_{c c} \frac{f_{c k}(t)}{\gamma_{C}} ; \alpha_{c c} \frac{C_{\max }}{\gamma_{C}}\right)
$$

avec :

$\alpha_{\text {cc }}$ coefficient qui dépend de la présence ou non d'une armature (armé $=1$, non armé $=0,8$ ) ;

$\gamma_{C}$ coefficient partiel dont la valeur est 1,5 à I'ELU fondamental et 1,2 à l'ELU accidentel;

$\mathrm{f}_{\mathrm{ck}}{ }^{*}$ résistance caractéristique à la compression du béton du coulis ou mortier d'une inclusion déterminée à partir de la formule suivante :

$$
f_{c k}^{*}=\inf \left(f_{c k}(t) ; C_{\text {max }} ; f_{c k}\right) \frac{1}{k_{1} k_{2}}
$$

avec :

$\mathrm{f}_{\mathrm{ck}}$ résistance caractéristique en compression du béton mesurée sur cylindres à 28 jours ;

$\mathrm{f}_{\mathrm{ck}}(\mathrm{t})$ résistance caractéristique en compression du béton mesurée sur cylindres au temps $t$;

$\mathrm{k}_{1}$ et $\mathrm{k}_{2}$ fonction de la méthode de forage et de l'élancement ;

$\mathrm{k}_{3}$ fonction du type d'ouvrage en cas de contrôle renforcé ou de procédures supplémentaires d'essais insitu ;

$\mathrm{C}_{\max }$ tient compte de la consistance qu'il est nécessaire de donner au béton, coulis ou mortier frais.

- Définition de $\mathrm{k}_{3}$

La valeur de $\mathrm{k}_{3}$ est définie dans le tableau suivant en fonction du type d'essai, du type de fondations et du nombre d'inclusions sous cette fondation.

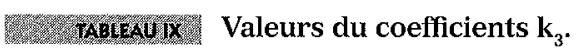

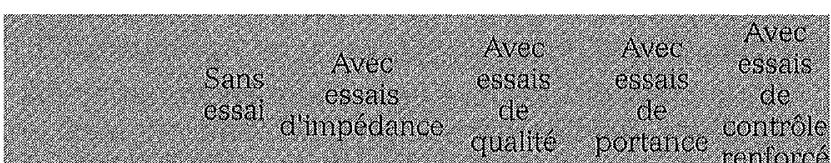

Domaine 1 :

(inclusions

nécessaires

à la stabilité)

Domaine 2 :

(inclusions

$$
\text { non }
$$

1,0

1,0

1,1

1,15

1,2

1,2

nécessaires

à la stabilité)
Essais de qualité à la charge de service ; essais de portance à 1,5 fois la charge de service avec paliers de fluage ; essais de contrôle renforcé au sens du DTU 13.2. La charge de service est définie comme la charge apportant la contrainte maximale à I'ELS. Les colonnes testées peuvent faire partie ou non de l'ouvrage.

- Taux de travail à l'ELS et à l'ELU

A l’ELU, la contrainte maximale de compression est limitée à la valeur de calcul $f_{c d}$ et la contrainte moyenne de compression sur la seule section comprimée est limitée forfaitairement à $7 \mathrm{MPa}$.

A l'ELS, la contrainte maximale de compression du béton est limitée à $0,6 \mathrm{f}_{c}{ }^{*}$ et la contrainte moyenne de compression sur la seule section comprimée de celui-ci limitée à min $\left(0,3 \mathrm{f}_{c}^{*} ; 0,45 \mathrm{f}_{c}\right)$.

Commentaire : En cas d'inclusions rigides réalisées en mortier ( $f_{C} \leq 15 \mathrm{MPa}$ ) ou en béton de sol (Jetgrouting, soil mixing..., $f_{c j}$ calculée en fonction des résultats obtenus à partir des essais d'écrasement), avec des dosages en ciment ou en liant inférieurs aux normes concernant les bétons, la contrainte moyenne sur la section comprimée s'obtient avec les coefficients $k_{1}, k_{2}$, et $k_{3}$ et limitée à la valeur de $5 \mathrm{MPa}$ en situation ELS ; la contrainte maximale en flexion composée est limitée à $10 \mathrm{MPa}$ en situation ELU.

\section{4.}

Inclusions métalliques

Pour les matériaux métalliques, les nuances et qualités du matériau sont celles de la NF EN 10025.

Commentaire : Prendre en compte les épaisseurs sacrificielles dues à la corrosion (cf. Fascicule 62-Titre $V$ [MELT, 1993])

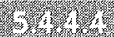

\section{Vérification en flexion composée et en cisaillement}

\section{5.}

Transmission des efforts horizontaux

Il convient de dimensionner le matelas de répartition de manière à limiter au maximum la transmission des efforts horizontaux aux inclusions rigides (effort horizontal, cisaillement...). L'intensité de ces efforts doit être évaluée ainsi que les conséquences sur les inclusions rigides en termes de contraintes maximum et minimum dans le matériau des inclusions.

L'objectif du calcul est de vérifier que les contraintes dans les inclusions rigides sont acceptables en considérant à la fois :

- la sollicitation verticale ;

- l'effort horizontal qui s'applique en tête d'inclusion; - le déplacement du sol sous l'effort horizontal appliqué par la fondation.

On propose la méthode simplifié suivante devant permettre de fournir un ordre de grandeur des résultats attendus.

- Méthode simplifiée

Les contraintes de cisaillement au niveau de la sousface de la semelle sont réparties en fonction du frottement sous la semelle donc au prorata de la répartition des contraintes verticales en compression: 
- respectivement $\sigma_{i}$ et $\tau_{\text {IR }}$ dans la colonne fictive surmontant l'inclusion rigide à travers le matelas d'une part;

- et $\sigma_{\mathrm{s}}$ et $\tau_{\mathrm{s}}$ hors emprise de cette colonne fictive d'autre part.

Elles ne s'appliquent donc que sur les sols ou sur les colonnes fictives travaillant en compression, en particulier en cas de moments de renversement s'appliquant sur la semelle.

Dans le cas d'une semelle soumise à un torseur $(Q$, $\mathrm{M}, \mathrm{H})$, chaque colonne sollicitée en compression à une valeur $\mathrm{Q}_{i}$ reprend donc en tête de colonne fictive un effort tranchant $h_{\mathrm{i}}$ tel que :

$$
\begin{gathered}
h_{\mathrm{i}}=H \mathrm{Q}_{\mathrm{i}} / \mathrm{Q} \\
\mathrm{h}_{\mathrm{s}}=\mathrm{H} \mathrm{Q}_{\mathrm{sem}} / \mathrm{Q} \text { avec } \mathrm{Q}=\mathrm{Q}_{\mathrm{sem}}+\Sigma \mathrm{Q}_{\mathrm{i}},
\end{gathered}
$$

avec $\mathrm{H}=\mathrm{h}_{\mathrm{s}}+\Sigma \mathrm{h}_{\mathrm{i}}$

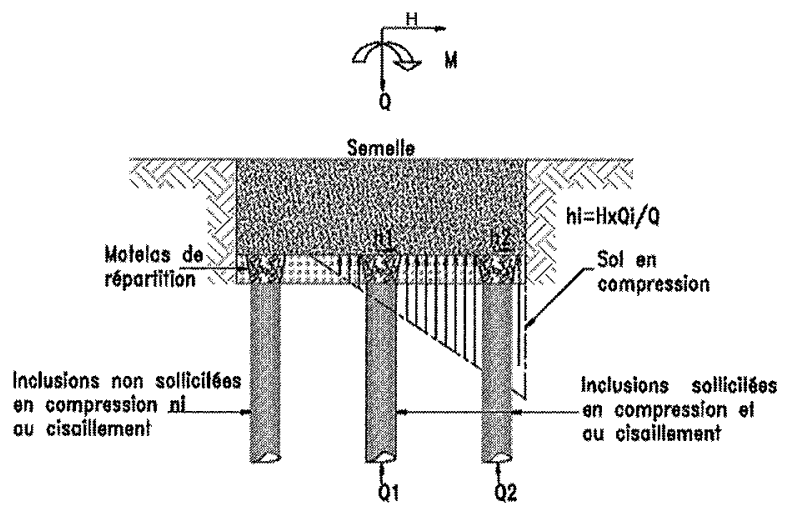

H6. 12. Schéma de répartition.

En fonction de l'épaisseur du matelas de répartition, les contraintes horizontales dans la colonne fictive diminuent avec la profondeur.

En première approche, on peut négliger cette diminution et calculer l'effort tranchant $h_{i}$ et le moment fléchissant $\mathrm{M}_{\mathrm{i}}$ induit dans la partie rigide de l'inclusion rigide selon le modèle élasto-plastique aux modules de réaction :

$h_{i}=\tau_{i} A_{\text {où }} A_{p}$ est la surface de l'inclusion rigide

$M_{i}=0,32 l_{o} h_{i}$ où $l_{o}$ est la longueur de transfert et dimensionner le ferraillage éventuel en fonction de $\mathrm{M}_{\mathrm{i}} / \mathrm{Q}_{\mathrm{i}}$ et de $\mathrm{h}_{\mathrm{i}}$.

Commentaire : A noter que cette méthode simplifiée est sécuritaire car elle ne prend pas en compte de diffusion des contraintes à travers le matelas de répartition et ne permet donc de donner qu'une valeur maximale des efforts horizontaux qui peuvent se transmettre aux inclusions.

- Méthodes aux éléments finis

Pour obtenir ces efforts de manière plus précise, on peut utiliser une approche plus complexe aux éléments finis (ou aux différences finies...), où l'on pourra :

- soit réaliser un modèle complet en 3D modélisant les inclusions rigides, le matelas de répartition, la semelle de fondation et leurs interfaces en appliquant le torseur total $(\mathrm{Q}, \mathrm{H}, \mathrm{M})$;

- soit par un modèle plus simple ;

- étape $n^{\circ} 1$ : on calcule avec un modèle axisymétrique les caractéristiques équivalentes du sol renforcé autour d'une inclusion rigide représentative (cylindre concentrique de sol autour de l'inclusion rigide, pour un maillage moyen), et la répartition des efforts de compression entre le sol et l'inclusion rigide,

- étape $n^{\circ} 2$ : faire un calcul 3D en remplaçant le sol renforcé par l'inclusion rigide par ce sol homogène équivalent en appliquant le torseur total (Q, H, M),

- étape $n^{\circ} 3$ : en déduire la rotation et le tassement maximum de la semelle, ainsi que la géométrie de la surface de sol entièrement comprimée sous la semelle afin de repérer les inclusions rigides en compression, et les contraintes de compression et cisaillement au droit de la maille la plus défavorable (de surface $\mathrm{S}_{\mathrm{m}}$ ) au niveau de la base du matelas,

- étape $n^{\circ} 4$ : sur cette maille, on répartit l'effort vertical $\left(\mathrm{S}_{\mathrm{m}}{ }^{*}\right.$ contrainte moyenne en compression) selon le schéma de l'étape 1 , et on définit $\mathrm{Q}_{\mathrm{i}^{\prime}}$

- étape $n^{\circ} 5$ : on répartit l'effort horizontal au prorata des compressions.

Remarque : Ces méthodes aux éléments finis permettent en particulier de prendre en compte les diffusions d'efforts tranchant à travers le matelas de répartition.

Commentaire : Elles doivent être précédées obligatoirement par un prédimensionnement analytique.

\section{3.}

Vérifications en flexion composée et en cisaillement

On vérifiera alors pour chaque inclusion en compression l'effort tranchant $V_{i}$ et l'excentrement des efforts $M_{i} / Q_{i}$ de manière à déterminer la nécessité éventuelle de ferraillage (pour le cas des inclusions béton) en fonction de $V_{i}$ et de $M_{i} / Q_{i}$.

\section{- Flexion composée}

Les contraintes normales extrêmes dans les inclusions rigides sollicitées en flexion composée (effort axial $\mathrm{N}$ et moment de flexion $\mathrm{M}$ ) sont alors données par la formule suivante :

$$
\sigma=\frac{N}{S} \pm \frac{M}{I / v}
$$

où, en cas de surface entièrement comprimée,

$$
S=\pi \frac{B^{2}}{4} ; I=\pi \frac{B^{4}}{64} ; \nu=\frac{B}{2}
$$

\section{- Cisaillement à l'ELU}

Les contraintes de cisaillement dans les inclusions rigides sollicitées en effort tranchant sont alors données par les formules suivantes en fonction de l'effort tranchant maximum $V_{i}$.

- En absence d'armatures et pour une section entièrement comprimée

Pour les inclusions non armées, on doit vérifier à $l^{\prime} E L U$ la contrainte de cisaillement conformément à l'Eurocode 2 section 12, ramenée au diamètre selon les équations suivantes :

$$
\begin{aligned}
& \begin{array}{c}
\tau_{c p}=1,91 \mathrm{~V}_{\mathrm{i}} / \mathrm{B}^{2} \\
\tau_{\mathrm{cp}}<\mathrm{f}_{\mathrm{cvd}} \\
\sigma_{\mathrm{clim}}=\mathrm{f}_{\mathrm{cd}}-2\left[\mathrm{f}_{\mathrm{ctd}}\left(\mathrm{f}_{\mathrm{ctd}}+\mathrm{f}_{\mathrm{cd}}\right)\right]^{0,5} \\
\sigma_{\mathrm{cp}}=\mathrm{Q}_{\mathrm{i}} / \mathrm{S}_{\mathrm{col}}
\end{array} \\
& \mathrm{f}_{\mathrm{ctm}}=0,3\left[\mathrm{f}_{\mathrm{ck}}\right]^{(2 / 3)} \\
& \mathrm{f}_{\text {ctk } 0.05}=0,7 \mathrm{f}_{\mathrm{ctm}} \\
& f_{\text {ctd }}=\alpha_{\text {cpl }} f_{\text {ctk0 } 0.05} / 1,5 \text { avec } \alpha_{\text {cpl }}=0,8
\end{aligned}
$$$$
\mathrm{S}_{\text {col }}=\text { section comprimée de la colonne }
$$

$\mathrm{f}_{\mathrm{cd}}=$ résistance de calcul en compression 


$$
\mathrm{f}_{c \mathrm{~d}}=\min \left(0,8 \mathrm{f}_{\mathrm{c}}^{*} / 1,5 ; 0,8 \mathrm{f}_{\mathrm{c}} / 1,5\right)
$$

$\mathrm{f}_{\text {cvd }}=$ résistance de calcul en cisaillement comprimée

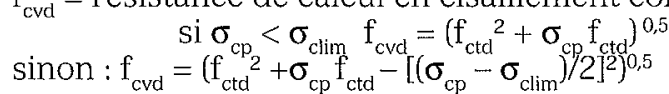

Conformément à l'Annexe nationale de l'Eurocode 2 et à la Norme d'application nationale à l'Eurocode 7 (NF P 94-262), aucun cisaillement n'est admissible quand le diamètre est inférieur à $400 \mathrm{~mm}$.

Conformément à la norme d'application nationale à l'Eurocode 7 (NF P 94-262), lorsque $\mathrm{N}_{\mathrm{ed}}$ dépasse à l'ELU $0,3 \mathrm{f}_{\mathrm{c}}^{*} \mathrm{~A}_{\mathrm{p}^{\prime}}$ on admet de prendre comme valeur de $\mathrm{f}_{\text {cyd }}$ :

$$
f_{c v d}=0,1\left[\left(f_{c t d}{ }^{2}+\sigma_{c p} \cdot f_{c t d}-\left[\left(\sigma_{c p}-\sigma_{c l i m}\right) / 2\right]^{2}\right)^{0,5}\right]
$$

On trouvera en annexe $\mathrm{E}$ les valeurs tabulées correspondantes en fonction de la résistance à la compression du matériau.

- En présence d'armatures

A l'ELU, on doit vérifier que $V_{i}<V_{R d, S}$ et que $\mathrm{V}_{\mathrm{i}}<\mathrm{V}_{\mathrm{Rd} \text {,max }}$

Les armatures à mettre en place sont calculées de la façon suivante :

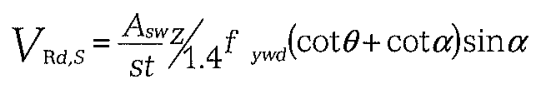

où $s_{t}$ est l'espacement des spires, $\alpha$ l'inclinaison des cerces, f $\gamma_{w d}$ est la résistance de l'acier $\left(=\mathrm{f}_{\mathrm{e}} / \gamma_{\mathrm{s}}\right)$, et où $\cot \theta$ peut varier entre 1 et 2,5.

Commentaire: On impose, dans le cadre de ces recommandations, que $\alpha=90^{\circ}$ et $\theta=45^{\circ}$.

L'effort tranchant ELU maxi repris par le béton est calculé de la façon suivante par la formule suivante :

$$
V_{R d, \max }=\alpha_{c W} \cdot\left(b_{W Z} / 1.4\right) V 1 f_{c d}(\cot \theta+\cot \alpha) /\left(1+\cot ^{2} \theta\right)
$$

où $\mathrm{z}$ est le bras de levier $(\mathrm{z}=7(\Phi-\mathrm{e}) / 8)$;

$\mathrm{b}_{\mathrm{w}}=\Phi ; \alpha_{c w}=1$.

\section{3re- 5145}

\section{Matelas de répartition}

Le transfert des charges depuis le massif de fondation se fait par l'intermédiaire d'un matelas de répartition. Ce matelas est obligatoirement interposé entre la fondation et la tête des inclusions.

Ce matelas permet également de limiter par effet de diffusion la transmission aux inclusions des efforts horizontaux et de cisaillement.

La couche de répartition de hauteur $\mathrm{H}_{\text {mat }}$ doit être mise en œuvre en fonction des critères de type « couche de forme ») ou (c routiers » (LCPC/SETRA 2000a et b), qu'elle soit constituée de matériaux frottants (graves naturelles) et/ou renforcée de liants (ciments, chaux, etc.).

Elle est caractérisée par des essais in situ de type ( routiers ) ou de type (" couche de forme ) (essais à la plaque...), ou par des essais géotechniques plus classiques (type essais pressiométriques ou pénétrométriques), ou par des essais de laboratoire (indice CBR ou IPI, mesures de la cohésion, de l'angle de frottement et de la teneur en eau, etc.).

Ces essais ont pour but de pouvoir vérifier la compacité en place des matériaux et de déterminer sa loi de comportement par l'estimation ses différents modules de déformations usuels (module pressiométrique si possible, module d'Young $E$ ou module cedométrique $\left.E_{\text {oed }}\right)$ et de cisaillement $\left(c^{\prime}, \theta^{\prime}\right)$ et de pouvoir calculer le tassement et la résistance ultime à la compression du matériau de cette couche et sa résistance au cisaillement.

Ses caractéristiques géo-mécaniques, par exemple le module EV2, et l'épaisseur de ce matelas, sont fonction de l'étude de dimensionnement du système de fondation. Elles dépendent des caractéristiques du sol en place, du diamètre, de l'espacement et de la profondeur des colonnes, du type et de l'intensité des charges et de la rigidité de la structure projetée.

Généralement cette couche de répartition est constituée d'au moins $40 \mathrm{~cm}$ de matériaux:

- de remblai granulaire en grave naturelle ;

- par exemple de classe D1, D2 ou D3 ou R suivant LCPC/SETRA (200a et b) (ou NFP 11-300),

- compactée à $95 \%$ de l'Optimum Proctot Modifié (OPM),

- ce qui lui conférera un module de déformation (équivalent à un module EV2) de l'ordre de $50 \mathrm{MPa}$, un rapport EV2/EV1 $<2,1$ et un angle de frottement de $40^{\circ}$ pour les matériaux concassées et $38^{\circ}$ pour les matériaux roulées ;

- de sols traités au liant dont les caractéristiques usuelles de cohésion et d'angle de frottement à prendre en compte pour les calculs sont $\mathrm{c}^{\prime}=50 \mathrm{kPa}$ et $\varphi^{\prime}=25^{\circ}$.

Concernant le comportement de l'inclusion, en tête, celle-ci émerge du sol médiocre et poinçonne le matelas de répartition. A ce niveau, le comportement de la face supérieure est assimilable à celui d'une plaque d'ancrage noyée à la profondeur $\mathrm{H}_{\text {mat }}$ (épaisseur du matelas) et sollicitée en traction vers le haut par une charge $Q_{1}(0)$ (égale à la charge en tête de l'inclusion).

Cette loi de comportement en tête de l'inclusion dépend des paramètres suivants :

- caractéristiques de déformation du matelas (module $\mathrm{d}^{\prime}$ Young et coefficient de Poisson) ;

- paramètres intrinsèques du matériau (cohésion et angle de frottement) ;

- état de confinement du matériau.

On prendra des dispositions constructives nécessaires pour que le matelas de répartition ne soit pas contaminé par le sol support (géotextile, couche anticontaminante...).

\subsection{0}

\section{Module de déformation}

Le module du matelas peut être calculé par corrélation des essais à la plaque, des essais CBR ou équivalent, ou par corrélation d'essais in situ (essais pressiométriques ou essais pénétrométriques).

- l'essai pressiométrique est à réserver pour des épaisseurs supérieures à $1 \mathrm{~m}$, le module de déformation $\mathrm{E}_{\mathrm{yst}}$ est alors assimilé à $E_{M} / \alpha / 1,5$ pour $v=0,33$;

- l'essai pénétrométrique est à réserver pour des épaisseurs supérieures à $0,8 \mathrm{~m}$; pour les matériaux granulaires on utilise alors la corrélation du type $\mathrm{E}_{\mathrm{y}} \approx 6 \mathrm{q}_{\mathrm{c}}$;

- les autres essais (plaque, CBR, IPI) doivent être privilégiés pour les autres cas; dans le cas de l'essai de plaque on considère $\mathrm{E}_{\mathrm{y}}=\mathrm{EV} 2$ (remblai granulaire en grave naturelle). 


\section{5.}

\section{Vérification du matelas}

Le mécanisme de rupture s'apparente à un poinçonnement de la tête dans le matelas.

Pour les matériaux traités aux liants hydrauliques, le mécanisme de ruine peut être lié à l'apparition de fissure (rupture par cisaillement ou rupture en traction).

\section{3.}

Calcul de la résistance limite en compression

On peut calculer la contrainte maximale mobilisable dans le matelas au niveau de la tête de l'inclusion à partir des paramètres de rupture du matériau : cohésion $c^{\prime}$ et de l'angle de frottement $\varphi^{\prime}$ selon le schéma de rupture de Prandtl sur l'épaisseur du matelas (Frossard et al., 2002, Berthelot et al., 2006, 2007 et 2011).

Le schéma de gauche de la figure 21 représente une répartition de contrainte en sous-face du radier et le schéma de droite représente la répartition simplifiée des contraintes $q_{1}$ et $q_{2}$ en sous-face du matelas.

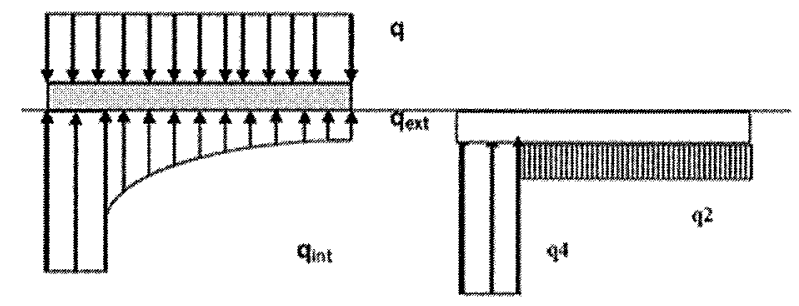

Coupure en sous-face radier

Sous-face matelas

16.13 Distribution des contraintes.

Le schéma de rupture du matelas de répartition au-dessus de la tête des inclusions peut être assimilé au mécanisme de Prandtl associant une zone en équilibre de poussée de Rankine (I) au-dessus de la tête de l'inclusion, une zone délimitée par un arc de spirale logarithmique (II) et une zone en équilibre de butée de Rankine à l'extérieur de la tête d'inclusion (III).

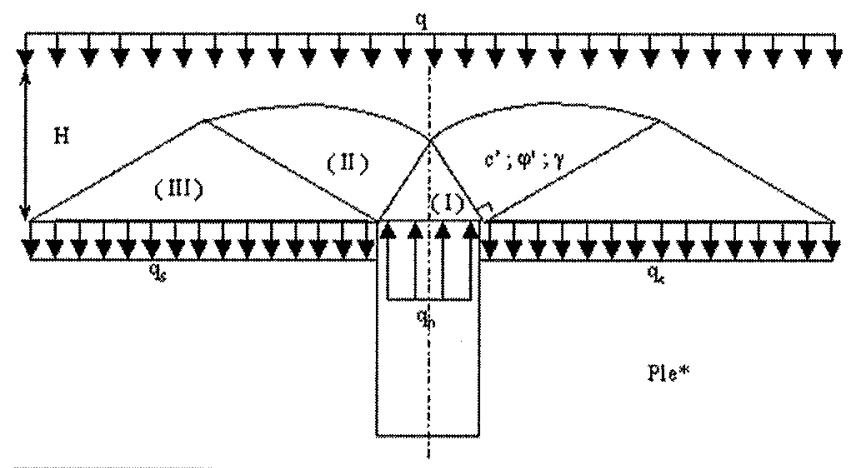

Schéma de rupture dans le matelas en spirale logarithmique.

La contrainte admissible dans le matelas en tête d'inclusion $q_{\text {mat }}$ est alors déterminée à partir de la contrainte appliquée sur le sol support $\mathrm{q}_{\text {ext }}$ (limitée à $\left.\mathrm{p}_{\mathrm{l}} / \gamma_{\text {sol }}\right)$ et des paramètres de rupture du matelas $\mathrm{c}^{\prime}$ et $\varphi^{\prime}$ par la relation suivante: $\mathrm{q}_{\text {mat;ELS }}=\min \left(3 \mathrm{MPa} ; \mathrm{q}_{1 ; \mathrm{ELS}}\right)$ à l'ELS

$\mathrm{q}_{\text {mat:ELU }}=\min \left(5 \mathrm{MPa} ; q_{1 ; E L U}\right)$ à l'ELU

où :

$$
q_{1}=N_{q} \times q_{2}+1.2 \times\left(\frac{C^{\prime}}{\gamma_{c}}\right) \times N_{C}
$$

$\mathrm{q}_{2}=\min \left(\mathrm{q}_{\mathrm{ext}}, \mathrm{p}_{1} / \gamma_{\mathrm{sol}}\right.$

$$
\begin{gathered}
N_{q}=\tan ^{2}\left(\pi / 4+\left(\frac{\varphi^{\prime}}{\gamma_{\varphi}}\right) / 2\right) \times e^{\pi \cdot \tan \left(\frac{\varphi^{\prime}}{\gamma_{\varphi}}\right)} \\
N_{c}=\left(N_{q}-1\right) \times \cot \left(\frac{\varphi^{\prime}}{\gamma_{\varphi}}\right) .
\end{gathered}
$$

et où on applique les coefficients de sécurité suivants :

$$
\begin{aligned}
& \gamma_{\text {sol }}=3 \text { à l'ELS et } 2 \text { à l'ELU ; } \\
& \gamma_{\varphi}=1,1 \text { à l'ELS et à l'ELU. }
\end{aligned}
$$

Exemple d'application : pour $40^{\circ}, \mathrm{N}_{\mathrm{q}}(40)=64,2$; $N_{c}(40)=75,4$

$\mathrm{N}_{\mathrm{q}}(40 / 1,1)=\mathrm{N}_{\mathrm{q}}(36)=37,8 ; \mathrm{N}_{\mathrm{c}}(40 / 1,1)=\mathrm{N}_{\mathrm{c}}(36)=50,6$ soit un coefficient de sécurité de $64,2 / 37,8=1,70 \operatorname{sur} \mathrm{N}_{\mathrm{q}}$ $\gamma_{c}=1,25$ à l'ELS et à l'ELU

soit un coefficient de sécurité de $1,25 * 75,4 / 50,5=1,80$.

Commentaire : On pourrait donc simplifier l'approche en retenant un coefficient de sécurité $\gamma_{\text {mat }}=1,75$.

- Influence de la hauteur du matelas

On se réfère au Projet national ASIRI (chapitre 4).

- Influence de la dimension du débord du matelas On se réfère au Projet national ASIRI (chapitre 4).

- Influence de la distance entre le bord de la semelle et le nu extérieur de l'inclusion

On se réfère au Projet national ASIRI (chapitre 4).

\section{6.)}

\section{Dispositions constructives}

Les dispositions des règlements en vigueur et celles du chapitre 5.1.2 s'appliquent et sont complétées dans les chapitres suivants.

\section{4.}

\section{Distance entre inclusions}

La distance minimale à respecter entre les axes des inclusions est de trois fois leur diamètre. Il appartiendra à l'entrepreneur de vérifier pendant la réalisation des travaux que, lors de l'exécution des colonnes, il n'y a pas de risque de détérioration des colonnes voisines, et si nécessaire l'ordre de leur exécution sera modifié.

\section{6.}

\section{Colonnes de confinement}

Dans le cas général, le maillage des inclusions couvre l'ensemble de la surface du massif de fondation et il n'y a pas lieu a priori, de prévoir des colonnes de confinement à l'extérieur de la semelle.

\section{S.r.t.}

\section{Couche de répartition}

Les inclusions rigides extérieures devront être implantées sur un cercle de diamètre égal au plus à :

$$
\varnothing-\left(h+\varnothing_{1}\right)
$$




\section{1.}

\section{Recépage Arases basses/plate-forme de travail/ matelas de répartition}

On préconise de réaliser les colonnes depuis une plate-forme de travail basse située au voisinage de la base de la fondation.

L'arase des inclusions rigides au niveau de la plate-forme est la meilleure façon de s'assurer des caractéristiques homogènes du matelas et d'un niveau précis d'arase béton permettant de limiter les variations d'épaisseur du matelas entre les têtes d'inclusions et la sous-face du radier.

\section{(3).}

Arase des inclusions au niveau de la plate-forme de travail

Le recompactage de la plate-forme de travail après la mise en place des inclusions ne peut alors être réalisé qu'après la mise en place d'au moins $25 \mathrm{~cm}$ de matériaux de type couche de forme afin d'éviter de compacter directement sur les têtes d'inclusions rigides.

\section{(1)}

Arase des inclusions au niveau de la base de la plate-forme de travail

Ces arases doivent être réalisées avec une tolérance maximum de $+1-5 \mathrm{~cm}$ pour éviter les variations locales inacceptables d'épaisseur du matelas de répartition. Il appartient à l'entrepreneur de mettre en cuvre les moyens nécessaires pour garantir ces tolérances.

On recommande en général la réalisation de ces arases à la suite de l'atelier de forage lorsque les colonnes sont encore ( fraîches ) à l'aide d'une mini-pelle. Les têtes de colonnes sont excavées jusqu'en sous-face de plate-forme ; le mélange foisonné de matériau constitutif de la plate-forme et de mortier de la colonne est ensuite remis en place dans le corps de plate-forme puis recompacté.

Le recompactage de la plate-forme de travail après la mise en place des inclusions ne peut être réalisé directement à ce niveau que dans la mesure où les arases sont réalisée à plus de $25 \mathrm{~cm}$ sous le niveau de la plateforme.

Dans le cas de matelas de répartition traités en place au-dessus de la tête des inclusions, l'entrepreneur devra justifier une cote d'arase précise compatible avec la tolérance de profondeur des machines de traitement.

Les terrassements dans l'encombrement des inclusions rigides non armées sont interdits : ce serait le cas par exemple d'une plate-forme de travail des inclusions rigides plus haute que la sous-face de la semelle.

\section{5}

\section{Vérifications et contrôle}

Les dispositions des règlements en vigueur et celles du chapitre 5.1.2 s'appliquent et sont complétées dans les chapitres suivants.

\section{1.}

\section{Matériau de l'inclusion}

Les fréquences de prélèvement des éprouvettes sont de 1 prélèvement pour $100 \mathrm{~m}^{3}$ de béton mis en place, avec un minimum de 1 prélèvement tous les 3 jours et de 1 prélèvement par éolienne, cela à raison de 6 éprouvettes par prélèvement.

\section{6.}

\section{Continuité de l'inclusion}

On vérifiera la continuité des inclusions réalisées - soit à raison de 1 inclusion sur 8 :

- par essai d'impédance,

- ou par essai de réflexion ;

- soit sur toutes les inclusions par enregistrements des paramètres de forages et de bétonnage.

\section{then}

\section{Matelas de répartition}

\section{1.}

Épaisseur.

On vérifie l'épaisseur du matelas par comparaison de relevés topographiques à raison de 3 points par éolienne.

\section{(1)}

Qualité

On recommande les essais de type « couche de forme n, essais de plaque, CBR ou IPI, avec éventuellement des mesures de $\varphi^{\prime}$, et de granulométrie.

Commentaire : En cas d'épaisseur de remblai supérieure à $80 \mathrm{~cm}$, on peut utiliser le pressiomètre ou le pénétromètre statique.

Les densités de ces différents types d'essais peuvent être les suivantes:

- essais de portance (plaque, $\mathrm{q}_{\mathrm{c}^{\prime}} \mathrm{p}_{1}$ ou CBR, au choix) : un minimum de 3 par massif et 3 par chantier ; - essais d'identification (granulométrie) et de caractérisation $\left(c^{\prime}, \varphi^{\prime}\right)$ : un minimum de 1 par chantier.

Commentaire : En cas de graves naturelles de bonne qualité et comme caractérisées ci-avant on peut ne pas effectuer les essais de caractérisation.

\section{Shis:}

\section{Plate-forme de travail/dispositions constructives}

Pendant et après les travaux de renforcement de sol, il convient de prendre des précautions pour éviter le cisaillement des inclusions (quand elles sont non armées).

Les risques de cisaillement de la partie supérieure de l'inclusion rigide peuvent en effet apparaître dans les cas suivants :

- circulation d'engins lourds de chantier directement sur les têtes d'inclusions rigides ;

- reprise de la plate-forme de travail après les travaux de renforcement de sol par des purges des matériaux. 


\section{Fondations sur pieux}

\section{5.}

\section{Description}

Un massif de fondation d'éolienne a généralement une forme polygonale assimilable à un massif circulaire de même surface et de diamètre $\Phi$. Sa base est généralement horizontale et se situe à une profondeur $\mathrm{h}$ du niveau de la plate-forme. Il s'appuie sur n pieux de diamètre $\Phi_{1}$, de profondeur $\mathrm{h}+\mathrm{L}$, situés sur un cercle de diamètre $\Phi_{2}$.

Le massif de fondation est considéré comme infiniment rigide.

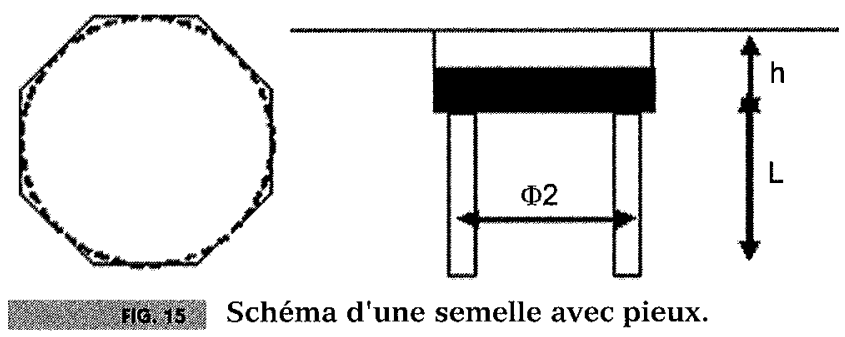

\section{1.}

\section{Données géotechniques}

On se référera au paragraphe 4.6 .

\section{5 .}

\section{Justifications}

\section{6.}

\section{Portance}

Les calculs de portance sont menés conformément aux règles en vigueur. Il n'est pas admis de traction dans les fondations profondes sous ELS quasi permanent (obtenu à partir principalement du cas de charge $\mathrm{DLC}_{\mathrm{QP}}$ ), à cause des effets cycliques.

\section{b.}

Cas particulier des micropieux

Les calculs de portance et de résistance de matériaux seront conformes aux règlements en vigueur.

Un essai de chargement est obligatoire à raison de 1 par 50 micropieux avec un minimum de 1 par chantier : il sera exécuté conformément aux règlements en vigueur.

Il n'est pas admis de traction dans les micropieux sous ELS quasi permanent (obtenu à partir principalement du cas de charge $\mathrm{DLC}_{\mathrm{OP}}$ à cause des effets cycliques).

\section{6.}

\section{Tassements et allongements}

- Tassement

Les calculs de tassement sont menés conformément à la méthode des courbes t-z (Frank/Zhao 1982, cf. fascicule 62-Titre V [MELT, 1993]) dans le domaine de déformation comprise du massif entre $10^{-2}$ et $10^{-3}$.

- Allongement

Pour le calcul de l'allongement, on ne retient comme produit ES du pieu que celui des aciers tant que la contrainte moyenne de traction du béton sur la section tendue du pieu est supérieure à $\mathrm{f}_{\mathrm{ct}} / \gamma_{\mathrm{s}}$. (en général valeur proche de 1,5 MPa).

\section{6.}

\section{Efforts horizontaux}

Les calculs vis-à-vis des efforts horizontaux sont menés conformément aux règles en vigueur (annexe $\mathrm{E}$ du Fascicule 62-Titre V [MELT, 1993]) dans le domaine de déformation comprise du massif entre $10^{-2}$ et $10^{-3}$.

Commentaire : Lorsque le massif n'est pas coulé en pleine fouille, la butée du sol sur le massif est négligée et les efforts horizontaux sous la sous-face de la semelle sont égaux à ceux appliqués en tête de semelle. Néanmoins, dans le cas où le massif est coulé en pleine fouille et sous réserve de la compatibilité des déformations basées sur la raideur en butée de la semelle d'une part et sur la raideur horizontale des pieux d'autre part, on pourra faire participer la butée de la semelle jusqu'à une valeur validée par le géotechnicien, qu'on limitera cependant à $30 \%$ de la valeur maximale de la poussée passive.

\section{S6ls}

\section{Rotation de l'ensemble}

La rotation sera calculée en fonction de la valeur du moment, des raideurs verticales et horizontales des pieux.

Il ne faut pas oublier de prendre en compte l'interaction horizontale sol-pieux et les effets de groupe entre pieux.

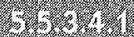

\section{Quand les pieux sont encastrés}

En effet, si les pieux sont encastrés, la rotation de l'ensemble induit des moments $M_{i}$ en tête de pieux en fonction :

- de leur rigidité EI ;

$\mathrm{EI}_{\text {entièrement comprimé }}>\mathrm{EI}$ partiellement comprimé $>\mathrm{EI}$ entièrement tendu - en fonction de la raideur du sol (c.-à-d. en fonction de $\left.\mathrm{K}_{\mathrm{s}}, \Phi\right)$;

- et donc de la valeur de la longueur de transfert $l_{0}$ selon la méthode aux modules de réaction).

Cela réduit la traction compression appliquée aux pieux.

Si on suppose que les pieux ont la même inertie, et par conséquent les mêmes raideurs en tête, à savoir $\mathrm{K}_{\mathrm{pv}}$ et $\mathrm{K}_{\mathrm{ph}}(\mathrm{MN} / \mathrm{m})$ respectivement en vertical et en horizontal, on peut mener les calculs suivants :

$$
\begin{aligned}
& \mathrm{J}=\mathrm{n} \Phi_{2}{ }^{2} / 8 \\
& \mathrm{~J} / \mathrm{V}=\mathrm{J} /\left(\Phi_{2} / 2\right)=\mathrm{n} \Phi_{2} / 4 \\
& \mathrm{Q}_{\text {max }}=\mathrm{M}^{\prime} /(\mathrm{J} / \mathrm{V})=4 \mathrm{M}^{\prime} / \mathrm{n} / \Phi_{2} \\
& \mathrm{M}^{\prime}=\mathrm{M}_{\mathrm{xy}}-\mathrm{n} \cdot \mathrm{M}_{\mathrm{i}} \\
& \mathrm{y}_{\max }=\mathrm{Q}_{\mathrm{max}} / \mathrm{K}_{\mathrm{pv}}^{\mathrm{xy}}=4 \mathrm{M}^{\prime} / \mathrm{n} / \Phi_{2} / \mathrm{K}_{\mathrm{pv}} \\
& \varphi=2 \mathrm{y}_{\max } / \Phi_{2}=8 \mathrm{M}^{\prime} / \mathrm{n} / \Phi_{2} / \mathrm{K}_{\mathrm{p}} / \Phi_{2} \\
& \rightarrow \mathrm{M}^{\prime} / \varphi=\Phi_{2} \mathrm{~K}_{\mathrm{pv}} \Phi_{2}, \mathrm{n} / 8
\end{aligned}
$$




$$
\begin{aligned}
& \mathrm{M}_{\mathrm{i}}=\varphi \mathrm{K}_{\mathrm{ph}} \Phi_{1} \mathrm{l}_{\mathrm{o}}^{3 / 4}=\left[8 \mathrm{M}^{\prime} / \mathrm{n} / \Phi_{2} / \mathrm{K}_{\mathrm{pv}} / \Phi_{2}\right] \mathrm{K}_{\mathrm{ph}} \Phi_{1} \mathrm{l}_{\mathrm{o}}^{3 / 4} \\
& \mathrm{M}_{\mathrm{i}}=\left[8\left(\mathrm{M}_{\mathrm{xy}}-\mathrm{n} \mathrm{M}_{\mathrm{i}}\right) / \mathrm{n} / \Phi_{2} / \mathrm{K}_{\mathrm{pv}} / \Phi_{2}\right] \mathrm{K}_{\mathrm{ph}} \Phi_{1} \cdot \mathrm{l}_{\mathrm{o}}{ }^{3} / 4 \\
& \text { En posant } \mathrm{c}=2 / \mathrm{n} / \Phi_{2} / \mathrm{K}_{\mathrm{pv}} / \Phi_{2} \mathrm{~K}_{\mathrm{ph}} \Phi_{1} \mathrm{lo}^{3} \\
& \mathrm{M}_{\mathrm{i}}=\mathrm{M}_{\mathrm{xy}} \mathrm{c} /(1+\mathrm{nc}) \\
& M^{\prime}=M_{x y}-n M_{i}=M_{x y}[1-n c /(1+n c)] \\
& \mathrm{K}_{\varphi}=\mathrm{M}_{\mathrm{xy}} / \varphi=\mathrm{M}^{\prime} / \mathrm{y}^{\prime} / \mathrm{d}=\Phi_{2} \mathrm{~K}_{\mathrm{pv}} \Phi_{2} \mathrm{n} / 8 / \mathrm{d} \\
& \rightarrow \mathrm{K}_{\varphi}=\Phi_{2} \mathrm{~K}_{\mathrm{pv}} \Phi_{2} \mathrm{n} / 8 \text { quand c est très grand } \\
& \rightarrow \mathrm{Q}_{\max }=4 \mathrm{M}_{\mathrm{xy}} / \mathrm{n} / \Phi_{2} / \mathrm{d}
\end{aligned}
$$

\section{6.}

Quand les pieux ne sont pas encastrés

Dans ce cas

$$
\begin{gathered}
\mathrm{d}=1 \\
\mathrm{M}_{\mathrm{i}}=0 \\
\mathrm{Q}_{\max }=4 \mathrm{M}_{\mathrm{xy}} / \mathrm{n} / \Phi_{2}
\end{gathered}
$$

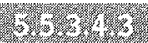

Charge maximale sur les pieux

Sous une charge centrée $Q$, la charge par pieu vaut :

$$
Q_{p}=F z / n
$$

On sera toujours entièrement comprimé si $Q_{p}>$ $\mathrm{Q}_{\max }$ soit si on vérifie que $\mathrm{M}_{\mathrm{xy}} / \mathrm{Fz}<\mathrm{n} \mathrm{d} \Phi_{2} / 4$.

La prise en compte de la réaction horizontale $d u$ sol sur les pieux quand ils sont encastrés :

- augmente $\mathrm{K}_{\phi}$;

- diminue la traction compression ;

- mais augmente les moments en tête de pieux.

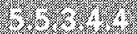

Conclusion pour le dimensionnement des pieux

A l'ELU, le dimensionnement géotechnique des pieux en traction pour $\mathrm{Q}_{\mathrm{p}}-\mathrm{Q}_{\max }$ sera calculé sans prendre en compte cette réaction horizontale.

En revanche, pour le ferraillage du pieu en flexion composée, et pour le ferraillage de la semelle, on prendra en compte l'effet de la réaction horizontale et donc la valeur des $M_{i}$.

\section{6.}

\section{Dispositions constructives}

Les dispositions des règlements en vigueur et celles du chapitre 5.1.2 s'appliquent et sont complétées dans les chapitres suivants.

\section{Armature des pieux en béton} més :

Dans le cas de pieux en béton, ces pieux sont ar-

- longitudinalement sur la hauteur nécessaire au calcul;

- toute hauteur en cas de traction-flexion;

- et transversalement conformément aux règlements en vigueur.

\section{6.}

\section{Vérification et et contrôle}

Les dispositions des règlements en vigueur s'appliquent et sont complétées dans les paragraphes suivants.

\section{ons}

\section{Béton des pieux}

Les fréquences de prélèvement des éprouvettes sont de 1 prélèvement pour $100 \mathrm{~m}^{3}$ de béton mis en place, avec un minimum de 1 prélèvement tous les 3 jours et de 1 prélèvement par éolienne, cela à raison de 6 éprouvettes par prélèvement.

Les caractéristiques du béton devront être conformes à la norme NF EN 206 et les normes d'exécutions de pieux.

\section{5}

\section{Armatures du radier}

Le radier sera dimensionné pour reprendre: - les moments d'encastrements $\mathrm{M}_{\mathrm{j}}$ si les pieux sont encastrés ;

- les moments et efforts tranchants générés par la transmission des efforts de compression ou de traction éventuelle dans les pieux;

- en fonction de la raideur respective de chaque pieu qui devra être justifiée par l'entrepreneur (en particulier en cas de terrain hétérogène).

\section{+1)}

\section{Continuité des pieux}

On vérifiera la continuité des pieux réalisés à raison de 1 pieu sur 8 :

- soit par essai d'impédance ;

- soit par essai de réflexion ;

et sur tous les pieux par enregistrements des paramètres de forages et de bétonnage.

\section{6}

\section{Fondations mixtes ou " composites"}

Le massif de fondation s'appuie à la fois sur le sol et sur n pieux de diamètre $\Phi_{1}$, de profondeur $h+L$, situés sur un ou plusieurs cercles de diamètre $\Phi_{2^{\prime}}$ (sans matelas de répartition entre la tête des pieux et le massif contrairement à la solution « fondations sur inclusions rigides $)$ ).

Le massif de fondation est considéré comme infiniment rigide.

Ces pieux peuvent être ou non reliés à la structure et ainsi reprendre ou non des efforts de traction. Quand ils sont reliés à la structure, il s'agit de " fondations mixtes » au sens d'O. Combarieu. Quand ils ne sont pas reliés à la structure, on les qualifiera sous le terme de ( fondations composites ).

Ils doivent ainsi être calculés sous compression, flexion composée et cisaillement, voire sous traction quand ils sont reliés à la structure. 


\section{6}

\section{Principes de fonctionnement}

La fondation mixte ou (c composite » peut être envisagée de deux façons différentes :

- comme une fondation classique sur pieux, la prise en compte de la présence de la semelle permettant une réduction du dimensionnement des pieux, au prix d'une légère augmentation du tassement d'ensemble ;

- comme une fondation directe sur le sol à laquelle on adjoint les pieux pour limiter le tassement.

Ces recommandations concernent ces deux types de fondations mixtes ou ( composites ); le nombre de pieux est quelconque, mais elles supposent que la semelle est infiniment rigide.

Une telle conception de fondation n'a d'intérêt que si le sol permet une mobilisation substantielle d'effort sous la semelle. Deux conditions au moins en limitent la pertinence :

- pieux reposant en pointe sur des sols très résistants, et semelle de répartition reposant sur des sols très compressibles ;

- tassement des sols superficiels supportant la semelle sous des actions extérieures telles que remblai, stockage, pompage...

La prise en compte de la mobilisation combinée des pieux et du sol sous la semelle peut ainsi permettre d'optimiser le dimensionnement global du massif de fondation (réduction du dimensionnement des pieux ou de la taille du massif de fondation).

\section{Singen}

\section{Description}

Les dénominations ( fondation mixte » ou « fondations composites »s'appliquent à l'ensemble « semelle et pieux » conçu et calculé en tenant compte des possibilités réelles de móbilisation simultanée des efforts dans le sol, par les pieux et la semelle (Combarieu, 1988 ; Borel, 2005).

Ces deux principes s'inscrivent en continuité entre la solution (c embase poids $\S 5.2$ ), la solution (c embase poids sur inclusion rigides $\$ 5.4$ ), et la solution "( fondations profondes $\S 5.5$ ). Elles se différencient fondamentalement de la solution « embase poids sur inclusions rigides " par l'absence de matelas, mais fonctionnent de la même façon par la mobilisation combinée du sol sous la semelle et des pieux.

On peut ainsi réussir à mobiliser à la fois les pieux et le sol sous la semelle, dans la mesure où leurs courbes de chargement respectives et tenant compte de l'interaction mutuelle sont compatibles.

Pour dimensionner un tel système, il est indispensable de faire systématiquement un calcul en déformation (type éléments finis ou calcul itératif...) modélisant l'ensemble des éléments de la fondation. Ce calcul doit être mené pour l'ensemble des cas de charges, de manière à pouvoir combiner les réactions respectives du sol et des pieux dans chacun des cas.

Ce modèle doit exploiter des lois de comportement évoluées des terrains et de toutes les interfaces et doit être capable de décrire le comportement du système sur l'ensemble de la plage de fonctionnement jusqu'à l'approche de la rupture.
Ce type de solution faisant participer le sol à la reprise des efforts et des déformations, des études de sensibilité sur les paramètres de sol sont également nécessaires systématiquement.

Commentaire : Une étude du comportement de l'ouvrage est indispensable pour ce type de solution. Elle doit obligatoirement prendre en compte les efforts verticaux, les efforts horizontaux et les moments de renversement en prolongement des méthodes de dimensionnement prenant en compte le chargement vertical centré (Combarieu, 1988) et celles prenant en compte les efforts horizontaux (Borel, 2005).

\section{5.}

\section{Données géotechniques}

Pour justifier la portance et pour calculer les tassements et la rotation de ce type de fondation, on rappelle que les données géotechniques doivent combiner :

- les exigences du chapitre 5.1.2 sur les embases poids d'une part ;

- les exigences propres aux calculs de la portance des pieux d'autre part, à savoir une connaissance du sol sur une hauteur égale à $\mathrm{L}+\max \left(5 \mathrm{~m} ; 7 \Phi_{1}\right)$.

Les études géotechniques successives doivent permettre de donner une coupe type, avec par couche sensiblement homogène, l'ensemble des paramètres de sol listés au $\$ 4.6 .1$ et 4.6.2.

Le géotechnicien doit aussi donner La valeur de $p_{1 e}$ * et de $q_{c e}$ sous la semelle, les raideurs $K_{v^{\prime}} K_{x^{\prime}} K_{y^{\prime}} K_{z}$ et $K_{\varphi}$ du sol à CT et à LT sous cette semelle et les valeurs de $G$ à prendre en compte pour le calcul en très petite déformation $\left(10^{-3}\right.$ à $\left.10^{-5}\right)$ doivent être aussi données dans le cadre d'une étude géotechnique.

\section{Stron}

\section{Justification des pieux}

\section{1..}

\section{Calcul des déformations et de la répartition des charges}

Le calcul des déformations et de la répartition de charge est indispensable au dimensionnement d'une fondation mixte ou d'une ( fondation composite ).

Ce calcul est basé sur les expressions qui relient les efforts unitaires aux déformations pour les différents éléments de la fondation. Il consiste, pour une charge appliquée à la fondation, à déterminer les efforts repris respectivement par la semelle, la surface latérale et la pointe des pieux et à calculer le tassement en tête de la fondation. On peut ainsi tracer la courbe charge-tassement pour celle-ci, et vérifier pour tout les cas de charge (ELS et ELU), le niveau de mobilisation de chacune des composantes vis-à-vis des charges limites correspondantes, ou de la charge intrinsèque des pieux.

La figure 16 représente pour une valeur $w_{s}(0)$ de l'enfoncement de la semelle, les tassements respectifs en fonction de la profondeur $\mathrm{z}$, du sol et des pieux avec l'hypothèse de compressibilité ou non de ceux-ci.

Sur la partie haute des pieux, sous la semelle, le tassement relatif $\mathrm{wr}(\mathrm{z})$ est toujours très faible. Il convient donc de considérer que le frottement mobilisable $t(z)$ peut éventuellement être négligé sur une longueur 


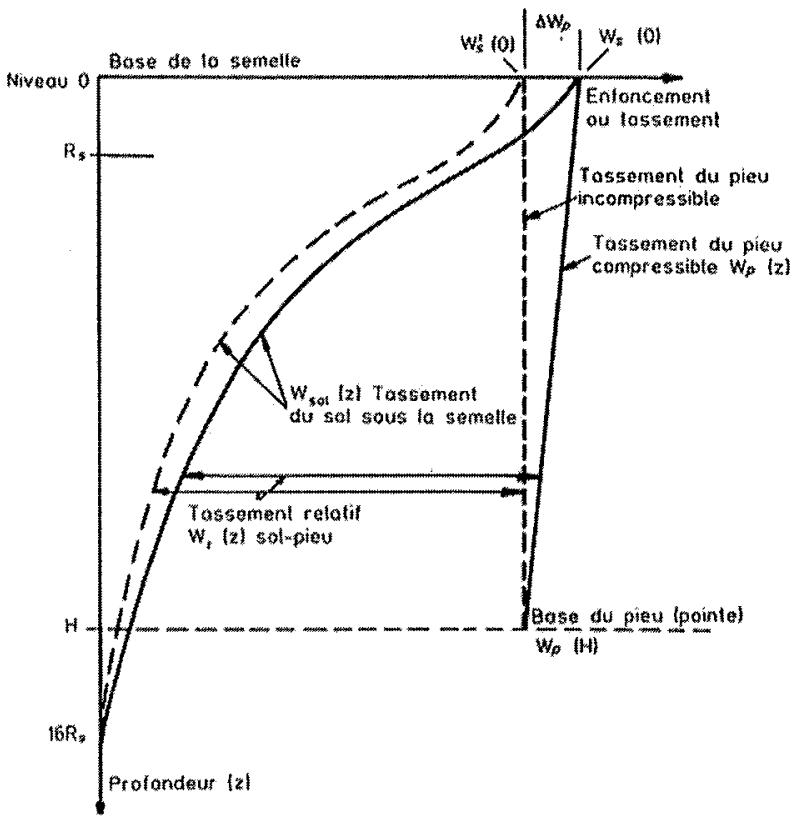

Ilc. 16. Tassement sol et éléments de fondation.

$\mathrm{R}_{\mathrm{s}}=\Phi / 2$ Pour le calcul de la portance, on recommande de neutraliser ce frottement sur $R_{s} / 2$.

Le frottement mobilisé sous $\mathrm{R}_{\mathrm{s}} / 2$ est calculé en fonction du déplacement relatif du pieu par rapport au sol.

Les calculs du tassement des pieux sont menés conformément aux règles en vigueur selon la méthode décrite par Combarieu (1988).

Pour le calcul de l'allongement des pieux lorsqu'ils sont reliés à la structure, on ne retient comme produit ES du pieu que celui des aciers tant que la contrainte moyenne de traction du béton sur la section tendue du pieu est supérieure à $f_{c t} / \gamma_{s}$.

Prendre en compte les raideurs croisées des deux composants (sol sous semelle et pieux) ainsi que l'interaction horizontale et le moment appliqué au massif nécessite des calculs aux éléments finis en 3D ou des calculs itératifs qui doivent mettre en exergue :

- le frottement mobilisé le long du fût des pieux;

- la rotation du massif ;

- la contrainte mobilisée sur le sol sous la semelle;

- la charge appliquée en compression ou en traction pour chacun des pieux ;

- les moments en tête des pieux à reprendre dans le radier lorsque les pieux y sont encastrés ;

- les efforts horizontaux appliqués à chacun des pieux.

Ce modèle doit exploiter des lois de comportement évoluées des terrains et de toutes les interfaces et doit être capable de décrire le comportement du système sur l'ensemble de la plage de fonctionnement jusqu'à l'approche de la rupture.

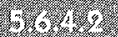

\section{Vérification de la portance du sol renforcé}

Dans tous les cas de charges ELS et ELU :

- on calcule $\mathrm{S}_{\text {comp }}, \sigma_{\max } \sigma_{\min }$ et $\mathrm{q}_{\text {ref }}=\left(3 \sigma_{\max }+\sigma_{\min }\right) / 4$ conformément au $\S 3.5 .2$ en intégrant en plus la reaction (positive ou négative) de chaque pieu à partir de calculs itératifs;
- on doit vérifier les critères de portance globale et de portance locale décrits aux paragraphes suivants.

Commentaire : Les picux situés dans la partie fictive du sol entièrement comprimée telle que dessinée en annexe $B$ travaillent en compression alors que le reste des pieux travaille en traction s'ils sont reliés à la structure.

\section{5.}

Critère de portance globale

Le critère suivant de portance globale est vérifié pour tous les cas de charges ELS et ELU avec :

$$
\begin{aligned}
& \mathrm{q}_{\text {solELS }}>\left(\mathrm{q}_{\text {refELS }} S_{\text {ref }}-n Q_{\text {pieuELS }}\right) /\left(S_{\text {ref }}-n A_{p}\right) \\
& q_{\text {solELU }}>\left(q_{\text {refELU }} S_{\text {ref }}-n Q_{\text {pieuELU }}\right) /\left(S_{\text {ref }}-n A_{p}\right)
\end{aligned}
$$

- la valeur limite de $Q_{\text {pieu }}$ à considérer est définie en référence au terme de pointe $R_{b}$ et au terme de frottement $\mathrm{R}_{\mathrm{s}}$.

$\mathrm{Q}_{\text {pieuELS }}=\min \left[\mathrm{A}_{\mathrm{p}} 0,3 \mathrm{f}_{\mathrm{c}^{*}} ;\left(\mathrm{R}_{\mathrm{b}} / \gamma_{\mathrm{b}}+\mathrm{R}_{\mathrm{s}} / \gamma_{\mathrm{s}}\right)\right]$

$\mathrm{Q}_{\text {pieuELU }}=\min \left[\mathrm{A}_{\mathrm{p}} \mathrm{a}_{\mathrm{cc}} \mathrm{f}_{\mathrm{c}} / 1,5 ;\left(\mathrm{R}_{\mathrm{b}} / \gamma_{\mathrm{b}}+\mathrm{R}_{\mathrm{s}} / \gamma_{\mathrm{s}}\right)\right]$

avec les méthodes pressiométriques ou pénétrométriques, on utilise respectivement les équations suivantes:

$$
\begin{aligned}
& q_{\text {solELU }}=\mathrm{k}_{\mathrm{p}} \mathrm{p}_{\mathrm{le}} / \gamma_{\text {solELU }}+\mathrm{q}_{\mathrm{o}}^{\prime} \text { ou } \mathrm{q}_{\text {solELU }}=\mathrm{k}_{\mathrm{c}} \mathrm{q}_{\mathrm{ce}} / \gamma_{\text {solELU }}+\mathrm{q}_{\mathrm{o}}^{\prime} \\
& \mathrm{q}_{\text {solELS }}=\mathrm{k}_{\mathrm{p}} \mathrm{p}_{\mathrm{le}} / \gamma_{\text {solELS }}+\mathrm{q}_{\mathrm{o}}^{\prime} \text { ou } \mathrm{q}_{\text {soleLS }}=\mathrm{k}_{\mathrm{c}} \mathrm{q}_{\mathrm{ce}} / \gamma_{\text {solELS }}+\mathrm{q}_{\mathrm{o}}^{\prime} \\
& \text { avec } \gamma_{\text {sol }} \gamma_{\mathrm{b}} \text { et } \gamma_{\mathrm{s}} \text { conforme aux reglements en vigueur. }
\end{aligned}
$$

Commentaire : Actuellement les valeurs sont les suivantes.

TABLARX Coefficients de sécurité proposés.

\begin{tabular}{ccccc}
\hline & & & & \\
\hline \\
ELS QP & pieu refoulé & 2,00 & 2,00 & 3,00 \\
& pieu foré & 2,80 & 2,00 & 3,00 \\
\hline \multirow{2}{*}{ ELS Rare } & pieu refoulé & 1,57 & 1,57 & 3,00 \\
& pieu foré & 2,20 & 1,57 & 3,00 \\
\hline ELU & & 1,40 & 1,40 & 2,00 \\
\hline
\end{tabular}

Compléments : Dans la limite de la vérification de la capacité portante globale du système avec l'application des coefficients de sécurité ci-dessus, il peut apparaitre lors des calculs de la répartition des contraintes entre le sol et les pieux que des valeurs limites de certains des éléments résistants (sol sous la semelle, frottement de l'inclusion et pointe du pieu) soient atteintes sans être dépassées (coefficient partiel de sécurité de 1 en fonctionnement).

Commentaire : Ces valeurs seront à actualiser dès la parution de la Norme d'Application nationale à l'Eurocode 7 NF 94-262.

\section{3.}

Critère de portance locale

On doit vérifier à partir des calculs itératifs de répartition de charges tels qu'explicités précédemment (§ 5.6.2) et pour tous les cas de charges (ELS et ELU) :

- que le critère suivant de portance locale du sol est vérifié avec:

$$
\begin{aligned}
& q_{\text {solELS }}>\sigma_{\operatorname{maxELS}} \\
& q_{\text {solELU }}>\sigma_{\text {maxELU }}
\end{aligned}
$$

où $\sigma_{\max }$ est la contrainte maximale reprise par le sol sous la semelle. 
Avec les méthodes pressiométriques ou pénétrométriques, on utilise respectivement les équations suivantes :

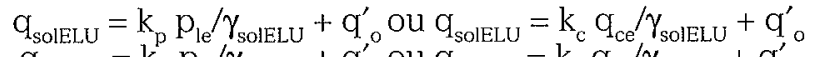$$
\mathrm{q}_{\text {solELS }}=\mathrm{k}_{\mathrm{p}} \mathrm{p}_{\mathrm{le}} / \gamma_{\text {solELS }}+\mathrm{q}_{\mathrm{o}}^{\prime} \text { ou } \mathrm{q}_{\text {solELS }}=\mathrm{k}_{\mathrm{c}} \mathrm{q}_{\mathrm{ce}} / \gamma_{\text {solELS }}+\mathrm{q}_{\mathrm{o}}^{\prime}
$$

- que pour chaque pieu du système, les contraintes calculées à partir des calculs itératifs de répartition de charges restent admissibles.

$$
\begin{gathered}
Q_{\text {pieuELS }}=\min \left[A_{p} 0,3 f_{c}^{*} ;\left(R_{b} / \gamma_{b}+R_{s} / \gamma_{s}\right)\right] \\
Q_{\text {pieuELU }}=\min \left[A_{p} a_{c c} f_{c}^{*} / 1,5 ;\left(R_{b} / \gamma_{b}+R_{s} / \gamma_{s}\right)\right]
\end{gathered}
$$

avec $\gamma_{b}$ et $\gamma_{s}$ conforme aux règlements en vigueur pour les pieux.

Compléments : Dans la limite de la vérification de la capacité portante locale du système avec l'application des coefficients de sécurité ci-dessus, il peut apparấtre lors des calculs de la répartition des contraintes entre le sol et les pieux que des valeurs limites de certains des éléments résistants (frottement et pointe du pieu) soient atteintes sans être dépassées (coefficient partiel de sécurité de 1 en fonctionnement).

\section{5}

\section{Efforts horizontaux}

Quand les pieux sont liaisonnés à la structure, les efforts horizontaux appliqués par pieu sont répartis au prorata de leurs raideurs horizontales.

Quand les pieux ne sont pas reliés à la structure, les efforts horizontaux $\mathrm{H}_{i}$ sont répartis sur les seuls pieux en compression au prorata de leurs charges verticales $N_{i}$ en vérifiant que $N_{i}{ }^{*} \operatorname{tg} \varphi^{\prime}$ est supérieur à la charge horizontale $\mathrm{H}_{\mathrm{i}}$ calculée précédemment, où $\operatorname{tg} \varphi^{\prime}$ béton $=0,8$.

Commentaire : Le fait de négliger le frottement solsemelle constitue une sécurité de ce dernier mode de fonctionnement.

A défaut d'un tel calcul en déformation, on pourra négliger la participation du sol et répartir les efforts horizontaux sur les seuls pieux.

\section{5.}

Prise en compte de la butée

En général, on ne prend pas en compte la butée du sol, ni la cohésion du sol.

Commentaire : Les efforts horizontaux sous la sousface de la semelle sont donc égaux à ceux appliqués en tête de semelle. Néanmoins, dans le cas où le massif est coulé en pleine foulle, on pourra faire participer la butée de la semelle jusqu'à une valeur déterminée par le géotechnicien, qu'on limitera cependant à $30 \%$ de la valeur maximale de la poussée passive.

\section{5.}

\section{Dispositions constructives}

Les dispositions des règlements en vigueur et celles du chapitre 5.1.2 s'appliquent et sont complétées dans les chapitres suivants.

\section{(1)}

\section{Sol support de fondation}

Pour cette solution, il est impératif de prendre les dispositions nécessaires pour protéger le fond de fouille en cours de travaux.
Dans ce cadre, les pieux doivent impérativement être réalisés depuis le fond de fouille à partir d'une plate-forme de travail (couche d'assise) compatible avec la circulation des engins.

Cette couche d'assise est destinée ensuite à servir de sol support de fondation, pour assurer un contact homogène entre la sous-face de la semelle et le sol, et ainsi permettre le transfert des charges de la semelle vers le sol.

La plate-forme de travail de hauteur $\mathrm{H}_{\text {mat }}$ doit être mise en œuvre en fonction des critères de type « couche de forme » ou « routiers » (LCPC/SETRA 2000a et b), qu'elle soit constituée de matériaux frottants (graves naturelles) et/ou renforcée de liants (ciments, chaux, etc.).

Elle est caractérisée par des essais in situ de type ( routiers ) ou de type (c couche de forme ) (essais à la plaque...), ou par des essais géotechniques plus classiques (type essais pressiométriques ou pénétrométriques), ou par des essais de laboratoire (indice CBR ou IPI, mesures de la cohésion, de l'angle de frottement et de la teneur en eau, etc.).

Ces essais ont pour but de pouvoir vérifier la compacité en place des matériaux et de déterminer leurs loi de comportement par l'estimation des différents modules de déformations usuels (module pressiométrique si possible, module d'Young $\mathrm{E}$ ou module oedométrique $M$ ) et des caractéristiques de cisaillement $\left(c^{\prime}, \varphi^{\prime}\right)$ et de pouvoir calculer le tassement de cette couche et sa résistance au cisaillement.

Ses caractéristiques géomécaniques, par exemple le module EV2, et l'épaisseur de ce matelas, sont fonction de l'étude de dimensionnement du système de fondation. Elles dépendent des caractéristiques du sol en place.

Généralement cette couche de répartition est constituée d'au moins $40 \mathrm{~cm}$ de matériaux :

- de remblai granulaire en grave naturelle ;

- par exemple de classe D1, D2 ou D3 ou R suivant GTR92 (NFP 11-300),

- compactée à $95 \%$ de l'OPM,

- ce qui lui conférera un module de déformation (équivalent à un module EV2) de l'ordre de $50 \mathrm{MPa}$, un rapport EV2/EV1 $<2,1$ et un angle de frottement de $40^{\circ}$ pour les matériaux concassées et $38^{\circ}$ pour les matériaux roulées.

- de sols traités au liant dont les caractéristiques usuelles de cohésion et d'angle de frottement à prendre en compte pour les calculs sont $\mathrm{c}^{\prime}=50 \mathrm{kPa}$ et $\varphi^{\prime}=25^{\circ}$.

La couche d'assise sera mise en œuvre selon les règles de l'art avec les contrôles usuels de réception appliqués aux couches de formes sous dallage.

On réalise un béton de propreté le plus vite possible, après réception par le géotechnicien ou le maître d'œuvre. Cela nécessite obligatoirement au préalable la remise en état de cette couche d'assise avant le coulage de ce béton de propreté.

Il est très important d'éviter tout remaniement du fond de fouille et s'entourant des précautions superficielles traditionnelles (en particulier pour les pieux à la boue...).

Afin de répartir le mieux possible la concentration des efforts en périphérie de massif, un débord de matelas est nécessaire sur une largeur correspondant au minimum à max $\left(\mathrm{H}_{\mathrm{mat}} / 2 ; 0,5 \mathrm{~m}\right)$ au-delà du bord $\mathrm{du}$ massif et de la derniere rangée de pieux, correspondant à la largeur minimale pour assurer un compactage satisfaisant. 
On prendra des dispositions constructives nécessaires pour que cette couche d'assise ne soit pas contaminée par le sol support (géotextile, couche anti-contaminante...).

\section{8}

\section{Armatures des pieux} més :

Dans le cas de pieux en béton, ces pieux sont ar-

- longitudinalement sur la hauteur nécessaire au calcul avec un minimum de $4 \mathrm{~m}$;

- toute hauteur en cas de traction-flexion;

- et transversalement conformément aux règlements en vigueur pour les pieux.

\section{6.}

\section{Pieux en traction}

Il n'est pas admis de traction dans les pieux en ELS quasi permanent.

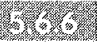

\section{Vérifications et contrôle}

Les dispositions des règlements en vigueur et celles du §5.1.2 s'appliquent et sont complétées dans les chapitres suivants.

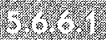

\section{Portance et altération du toit de l'excavation}

On se référera au $\$ 5.1 .3$.

\section{5}

\section{Couche d'assise}

\section{1.}

Épaisseur

On vérifie l'épaisseur du matelas par comparaison de relevés topographiques à raison de 3 points par éolienne.

\section{45}

Qualité

On recommande les essais de type « couche de forme m, essais de plaque, CBR ou IPI, ainsi que des mesures de $\varphi^{\prime}$, et de granulométrie.
Commentaire : En cas d'épaisseur de remblai supérieure à $80 \mathrm{~cm}$, on peut utiliser le pressiomètre ou le pénétromètre statique.

Les densités de ces différents types d'essais peuvent être les suivantes :

- essais de portance (plaque, $\mathrm{q}$, $\mathrm{p}_{1}$ ou CBR, au choix);

- un minimum de 3 par massif et 3 par chantier,

- essais d'identification (granulométrie) et/ou de caractérisation $\left(c^{\prime}, \varphi^{\prime}\right)$;

- un minimum de 1 par chantier.

Commentaire : en cas de graves naturelles de bonne qualité et comme caractérisées ci-avant on peut ne pas effectuer les essais de caractérisation.

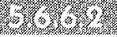

\section{Armatures du radier}

Le radier sera dimensionné pour reprendre :

- les moments d'encastrements $M_{i}$. des pieux quand ils sont encastrés;

- le poinçonnement des pieux dans le massif ;

- les efforts verticaux (compression, traction), les moments et efforts tranchants générés par la transmission des efforts de compression ou de traction éventuelle dans les pieux;

- en fonction de la raideur respective de chaque pieu et du sol sous la semelle qui devra être justifié par l'entrepreneur (en particulier en cas de terrain hétérogène).

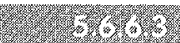

\section{Béton de pieux}

Les fréquences de prélèvement des éprouvettes sont de 1 prélèvement pour $100 \mathrm{~m}^{3}$ de béton mis en place dans les pieux, avec un minimum de 1 prélèvement tous les 3 jours et de 1 prélèvement par site, cela à raison de 6 éprouvettes par prélèvement.

Les caractéristiques du béton devront être conformes à la NF EN 206 et les normes d'exécution des pieux.

\section{2)}

\section{Continuité}

On vérifiera la continuité des pieux réalisés à raison de 1 pieu sur 8 :

- soit par essai d'impédance ;

- soit par essai de réflexion;

- et sur tous les pieux par enregistrements des paramètres de forages et de bétonnage (ou d'injection) en cas de pieux type tarière creuse, vissés moulés ou micropieux. 


\section{Annexe A (informative)}

\section{Lexique \\ A.1. Action géotechnique \\ L'action géotechnique est définie comme l'action transmise à la structure par le terrain, un remblai, une masse d'eau ou l'eau souterraine.}

\section{A.2. Charge opérationnelle}

La charge opérationnelle (norme NF EN 61400-1) doit être égale à la valeur la plus élevée :

a) des charges au cours de la production normale d'électricité en moyennant sur la durée de vie ;

b) des charges au cours de l'arrêt d'urgence pour une vitesse de vent choisie de sorte que les charges avant l'arrêt soient égales à celles obtenues avec a).

\section{A.3. Maître de l'ouvrage}

Le maître de l'ouvrage (Loi n 85-704 \& norme NFP 94-500) est la personne morale (ou physique) [...] pour laquelle l'ouvrage est construit. Responsable principal de l'ouvrage, il remplit dans ce rôle une fonction d'intérêt général dont il ne peut se démettre.

\section{A.4. Maître d'œuvre}

Selon la NFP 94-500, le maître d'cuvre est la personne physique ou morale qui assure la conception et/ ou la direction et le contrôle des travaux d'exécution de l'ouvrage pour le compte du maître d'ouvrage.

\section{A.5. Contrôleur technique}

$$
\text { Cf. chapitre 2.1.2 et 2.2.4 }
$$

\section{A.6. Géotechnicien}

Le géotechnicien (NFP 94-500) est la personne physique ou morale qui réalise des prestations d'ingénierie géotechnique et/ou d'investigations géotechniques.

\section{A.7. Exploitant}

L'exploitant (Petit Larousse illustré, 1997) est la personne qui met en valeur un bien productif de richesse.

\section{A.8. Étude géotechnique}

La géotechnique constitue (NFP 94-500) l'ensemble des activités liées aux applications de la mécanique des sols, de la mécanique des roches et de la géologie de l'ingénieur. La géotechnique englobe l'étude des propriétés géotechniques des sols et de l'interaction entre les terrains et les ouvrages environnants d'une part, l'ouvrage objet de la prestation du fait de sa réalisation et/ou de son exploitation d'autre part.

La géotechnique s'appuie principalement sur les différentes sciences de la terre suivantes :

- la géologie ;

- l’hydrogéologie ;

- la mécanique des sols et des roches ;

- la rhéologie des géomatériaux;

- la géophysique ;

- la géodynamique ;

- la géochimie.

Les investigations géotechniques (NFP 94-500) regroupent l'ensemble des recherches et reconnaissances effectuées par l'utilisation des matériels de forages $^{(1)}$, sondages ${ }^{(2)}$, mesures et essais géotechniques in situ et en laboratoire, mis en œuvre pour recueillir des informations géologiques et géotechniques sur les sols et les roches d'un site, telles que leur nature, leur composition, leur structure et leur répartition spatiale, ainsi que leurs caractéristiques physiques et chimiques, géomécaniques et hydrogéologiques. Une panoplie d'essais permettent l'investigation géotechnique (cf. les normes NF P 94- Sols : reconnaissance et essais et l'Eurocode 7 NF EN 1997-2).

\section{A.9. Maîtrise d'œuvre}

La mission de maîtrise d'œuvre (Loi n 85-704) que le maître de l'ouvrage peut confier à une personne de droit privé ou à un groupement de personnes de droit privé doit permettre d'apporter une réponse architecturale, technique et économique au programme [...]. Pour la réalisation d'un ouvrage, la mission de maîtrise d'œuvre est distincte de celle d'entrepreneur.

\section{A.10. Contrôle technique}

Cf. chapitre 2.1.2 et 2.2.4.

\section{A.11. Divers}

Les ouvrages géotechniques (norme NFP 94-500) sont des ouvrages ou parties d'ouvrage assurant le transfert des interactions entre un ouvrage dans sa globalité et les terrains dans lequel il s'insère. Les ouvrages géotechniques sont notamment pour les plus courants et sans exhaustivité :

- les fondations (semelles, radiers, puits, pieux, barrettes, murs enterrés...);

- les soutènements par tout procédé (murs préfabriqués, murs coulés en place, béton projeté, inclusions, clouage, terre armée...);

- les ouvrages en terre (avec les matériaux du site ou extérieurs au site) et les aménagements de terrains par terrassement, havage, dragage (talutage, modification de pente, déblais, remblais, couches de forme, digues, barrages...) ;

- les ouvrages souterrains, avec ou sans dispositif de soutènement (puits, excavations, galeries, tunnels, chambres de stockage...);

- les ouvrages de drainage, d'épuisement et de pompage.......

Le rapport géotechnique (Fascicule 62, article A.2.2 [MELT, 1993], norme NF P94-500) est établi à partir des essais effectués, de l'expérience locale des terrains acquise en particulier lors de la réalisation de constructions antérieures et des différents types de mission.

Le sol (NFP 94-500) [...] est le terme générique employé en géotechnique pour désigner tout terrain naturel ou artificiel, susceptible d'être mobilisé par la construction d'un ouvrage, englobant les sols ou les roches en place ou en remblai. Il diffère tout à fait du sens littéral usuel dans lequel le sol ne désigne que la surface du terrain, le reste (sous-sol) étant constitué tout d'abord d'une couche superficielle plus ou moins épaisse (sol au sens de l'agriculture ou de la pédologie) résultant de l'altération des roches sous-jacentes, puis de l'ensemble des constituants minéraux de l'écorce terrestre qui sont des roches au sens géologique.

\footnotetext{
(1) Forage (NFP 94-500) : réalisation d'une excavation linéaire avec un outil et un procédé spécifiques aux techniques de percement. (2) Sondage (NFP 94-500) : exploration locale et méthodique d'un terrain à partir d'une excavation, d'un trou de forage, de la pénétration d'une sonde ou de l'utilisation de techniques physiques non ou peu destructives, pour en déterminer la nature, la structure ou effectuer des mesures de propriétés physiques ou chimiques, mécaniques ou hydrauliques.
} 


\section{Annexe B}

Développement sur le calcul de $\mathrm{q}_{\mathrm{ref}}$, définition de la ( lunule ») et de $S_{\text {ref }}$

B.1. Influence du \% de semelle entièrement comprimé sur le taux de travail maxi du sol

Pour lo tassemear d'une semelle soumise à un efiont vertical $Y$ sxcentrè, il est nécessate de détermiute la surfece comprimese at la contuainte mojeute qui s'y applique.

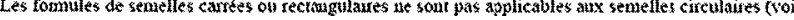
la forme de la zone comprimét hachurete du dessin eit.dessous)

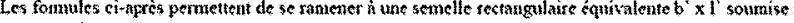
tule contrainte movente o $\mathrm{s}$.

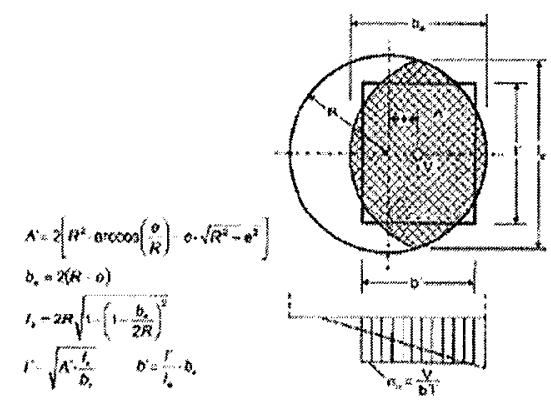

16.812

Pour une éolienne soumise au torseur $\left(\mathrm{M}_{\mathrm{ELU}} ; \mathrm{F}_{\mathrm{zELU}}\right)$, la surface de référence $S_{\text {ref }}$ est la zone hachurée de la figure ci-dessus (lunule : limitée par deux arcs de cercles symétriques par rapport à un axe situé à $\mathrm{e}=\mathrm{M}_{\mathrm{ELU}} / \mathrm{F}_{\mathrm{zELU}}$ du centre de l'éolienne).

\section{B.2. Définition de la zone entièrement comprimé}

\begin{tabular}{|c|c|c|c|c|}
\hline W & \multicolumn{4}{|c|}{$\begin{array}{l}\text { Contrainte sous la base d'une fondation } \\
\text { rectangulaire selon le modèle de Navier }\end{array}$} \\
\hline Tonctivin & 18xtentinte & (1979) & & (2) \\
\hline \multirow{2}{*}{$\begin{array}{l}\text { Rectangulaire } \\
\text { (BL) }\end{array}$} & $0 \leq c_{B} \leq \frac{B}{6}$ & $\frac{1}{B n}\left(1+6 \frac{w_{n}}{B}\right)$ & $\frac{1 \cdot}{B D}\left(1-6 \frac{x_{3}}{B}\right)$ & $\mathrm{BL}$ \\
\hline & $\frac{b}{6} \leq 4 \leq \frac{b}{2}$ & $\frac{1}{B H} \frac{4}{3\left(1-2 \frac{z_{a}}{B}\right)}$ & 0 & $1.5\left(1-2 \frac{e}{3}\right)(B L$ \\
\hline
\end{tabular}

B.3. Coefficient réducteur $i_{\text {dû }}$ à l'excentricité de la charge dans le cas d'une fondation sous charge verticale excentrée

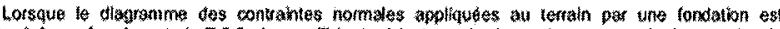

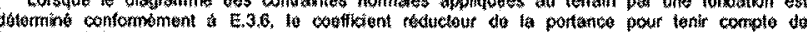

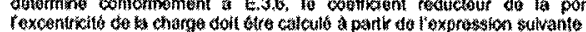

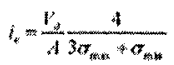

expression dans latavelle

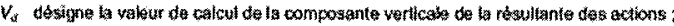

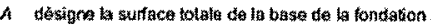

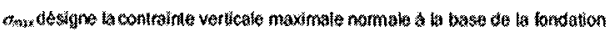

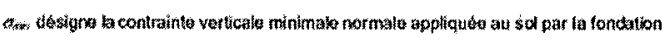

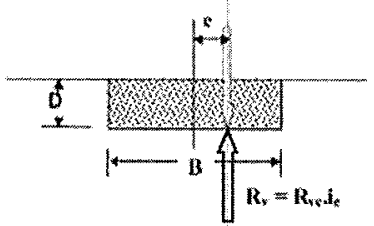

B.4. Définition de la surface comprimée $S_{\text {comp }}$ et de la surface de référence $S_{\text {ref }}$

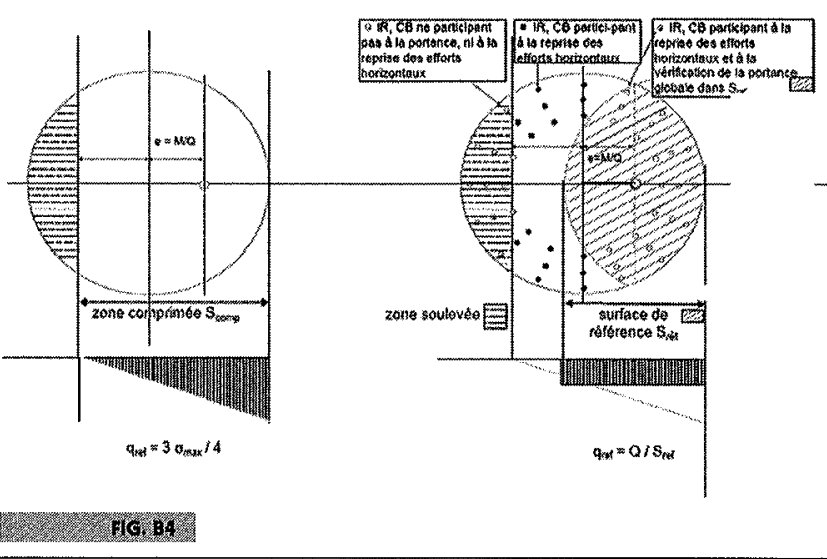

\section{Annexe $\mathrm{C}$}

Courbes de dégradation de $\mathrm{G}$ en fonction de la distorsion; extraits PS92 donnant les ordres de grandeurs de $V_{s}$

\section{C.1. Pour des matériaux argileux}

Modulus Degradation Curve (Vucetic \& Dobry, 1991)

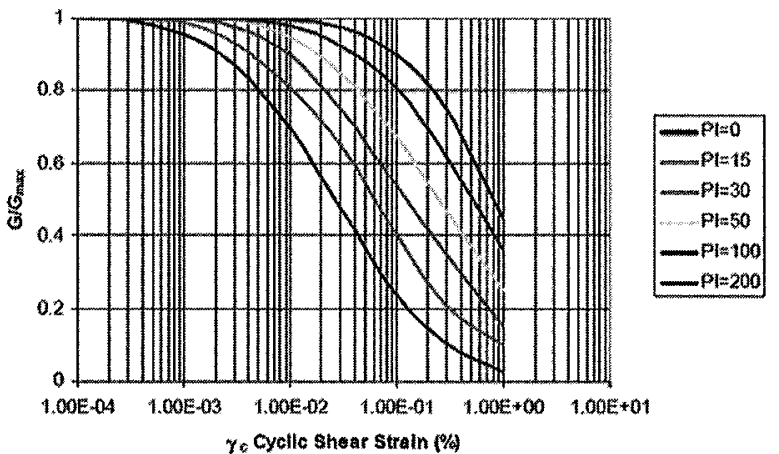

Modulus Degradation Curve (Seed at al, 1984)
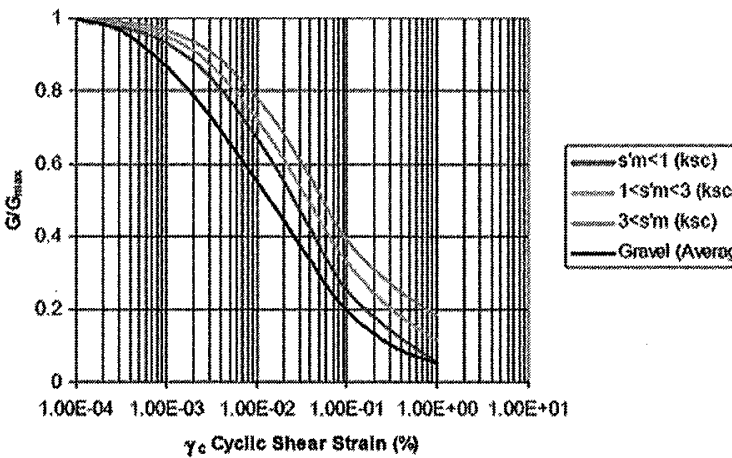

maxmen $3<\sin ^{2}(\mathrm{ksc})$

Gravel (Averag 


\section{C.2. Pour des matériaux granulaires}

Tableau 5.2.1 de la norme NFP 06013 [règles PS92].

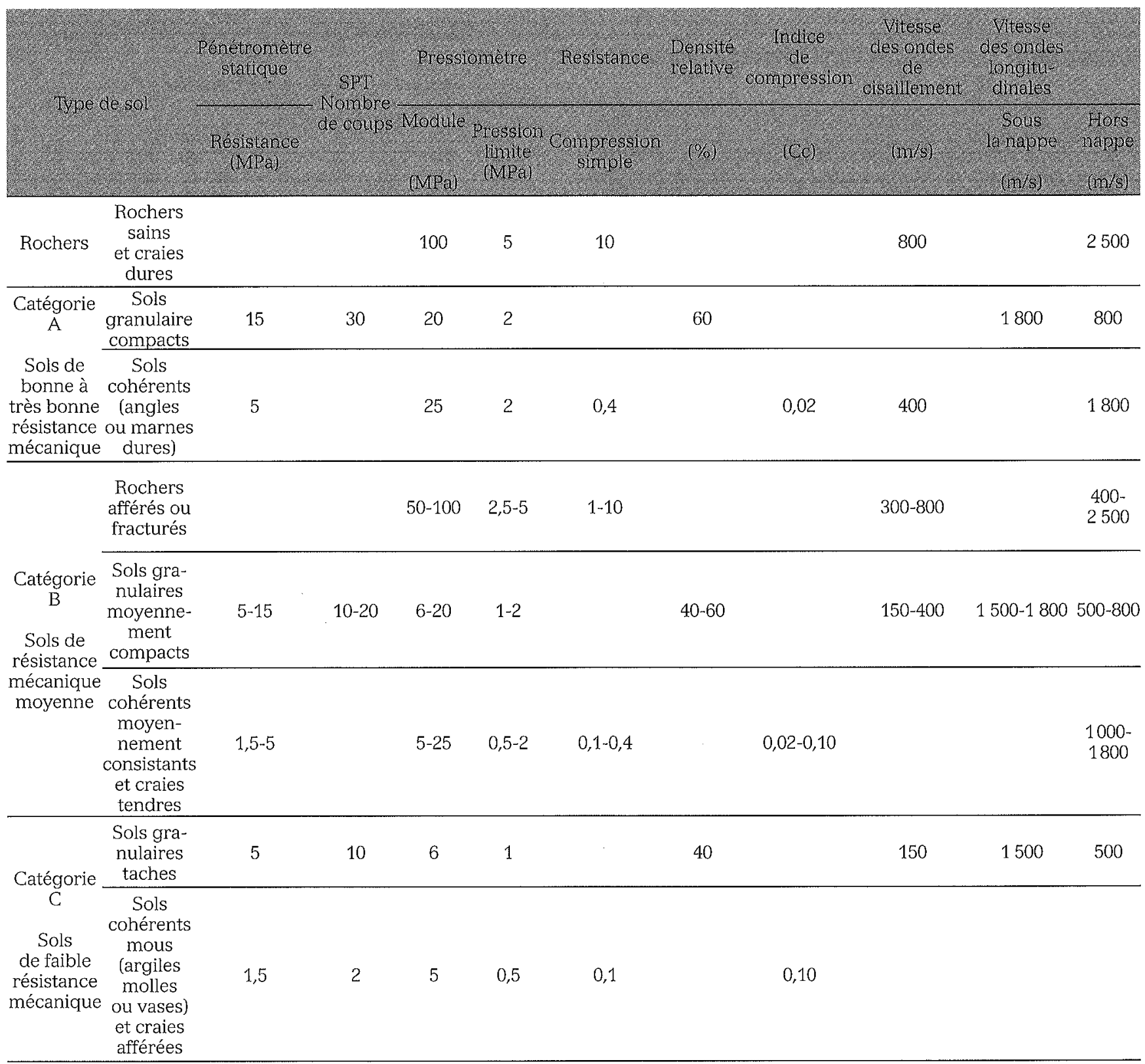




\section{Annexe D}

Calcul de $\mathrm{p}_{\mathrm{le}}$ selon la méthode de la semelle fictive

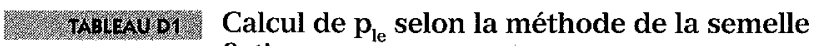
fictive.

\begin{tabular}{|c|c|c|c|c|c|}
\hline (2) & 10 & (1) & 6 & P. & $n^{3}$ \\
\hline 1,00 & 0,50 & 0,00 & 0,00 & 0,50 & \\
\hline 2,50 & 1,00 & 1,75 & 0,11 & 1,23 & \\
\hline 4,00 & 0,80 & 3,25 & 0,20 & 1,16 & \\
\hline 5,50 & 2,00 & 4,75 & 0,30 & 3,36 & \\
\hline 7,00 & 0,25 & 6,25 & 0,39 & 0,48 & 0,48 \\
\hline 8,50 & 1,00 & 7,75 & 0,48 & 2,20 & \\
\hline 10,00 & 1,20 & 9,25 & 0,58 & 2,99 & \\
\hline 11,50 & 2,00 & 10,75 & 0,67 & 5,59 & \\
\hline 13,00 & 2,20 & 12,25 & 0,77 & 6,86 & \\
\hline 14,50 & 0,20 & 13,75 & 0,86 & 0,69 & \\
\hline 16,00 & 1,00 & 15,25 & 0,95 & 3,81 & \\
\hline 17,50 & 1,00 & 16,75 & 1,05 & 4,19 & \\
\hline 19,00 & 1,20 & 18,25 & 1,14 & 5,50 & \\
\hline 20,50 & 0,20 & 19,75 & 1,23 & 1,00 & \\
\hline 22,00 & 1,00 & 21,25 & 1,33 & 5,42 & \\
\hline 23,50 & 1,00 & 22,75 & 1,42 & 5,87 & \\
\hline 25,00 & 1,00 & 24,25 & 1,52 & 6,33 & \\
\hline 26,50 & 1,00 & & & & \\
\hline 28,00 & 1,00 & & & & \\
\hline 29,50 & 1,00 & & & & \\
\hline
\end{tabular}

Remarque : si on avait mesuré une deuxième valeur de $0,25 \mathrm{MPa}$ à $8,5 \mathrm{~m}$ de profondeur, la valeur de $p_{1:}$ correspondante serait égale à 0,55 inférieure à la valeur de 0,48 calculée pour $p_{1}$ de $0,25 \mathrm{MPa}$ mesurée à $7 \mathrm{~m}$ de profondeur.

$N B$ : Une troisième valeur de $0,25 \mathrm{MPa}$ à $10 \mathrm{~m}$ de profondeur correspond aussi à une valeur de pli supérieure $(0,61 \mathrm{MPa})$. Lépaisseur de la couche caractérisée par $p_{1}=0,25 \mathrm{MPa} n^{\prime}$ aurait donc pas de conséquence sur le calcul de $p_{l e}$. En revanche, cette épaisseur joue un rôle primordial dans le calcul du tassement.

\section{Annexe E}

Valeurs tabulées de $\mathrm{f}_{\text {cvd }}$

Pour l'utilisation du tableau suivant, on prend $f_{c k}=f_{c}$ *

\begin{tabular}{|c|c|c|c|c|c|c|c|c|c|}
\hline f. & fost & 5 & 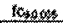 & feta & 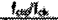 & $t_{0}$ & 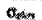 & 4 & tot \\
\hline & $\log +\mathrm{ten}, 5$ & $2 a^{3} \times \mathrm{x}^{3393}$ & $07 \mathrm{rtm}$ & 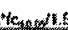 & & & & & \\
\hline 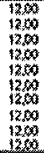 & 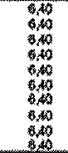 & 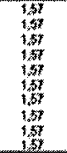 & 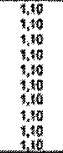 & 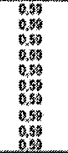 & 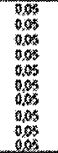 & 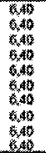 & 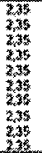 & 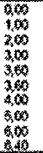 & 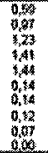 \\
\hline 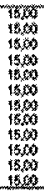 & 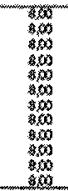 & 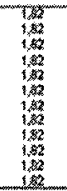 & 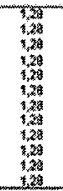 & 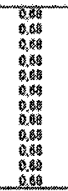 & 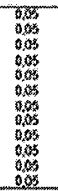 & 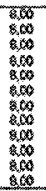 & 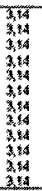 & 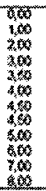 & 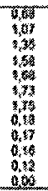 \\
\hline 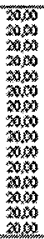 & 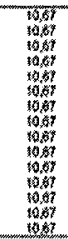 & 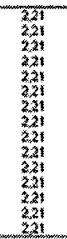 & 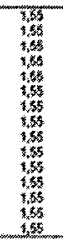 & 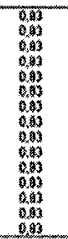 & 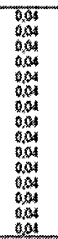 & 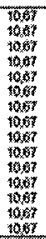 & 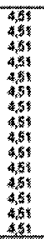 & 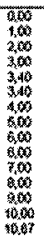 & 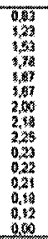 \\
\hline 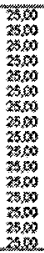 & 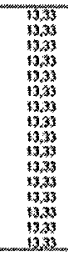 & 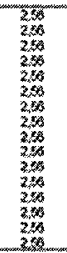 & 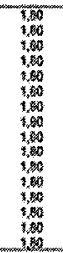 & 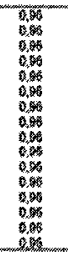 & 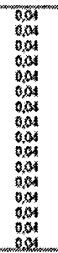 & 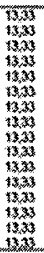 & 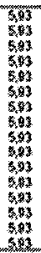 & 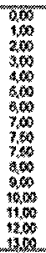 & 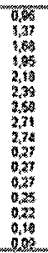 \\
\hline
\end{tabular}

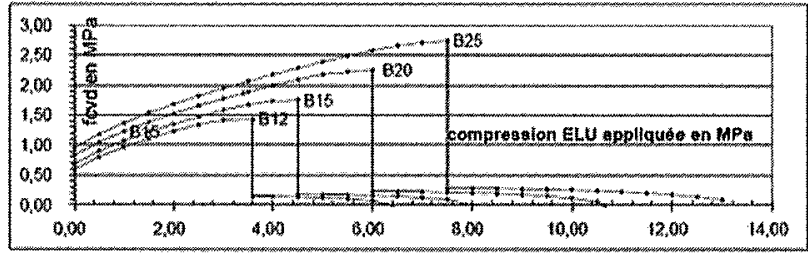




\section{Bibliographie}

\section{Reconnaissance géotechnique}

DTU 11.1. - Sondage des sols de fondation. Revue française de géotechnique, décembre 1968.

XP P94-010 - Sols : reconnaissance et essais - Glossaire géotechnique - Définitions - Notations - Symboles. (Soils: investigation and testing. Geotechnical glossary - Definitions - Notations Symbols), 24 p., 1996.

Baguelin F. - Rapport du sous-comité Symboles, Unités, Définitions. Comptes rendus du $I X^{e}$ congrès international de Mécanique des Sols et des Travaux de Fondations, Tokyo, 1977, p. 153-170.

Park, C.B., Miller, R.D., Xia, J. - Multichannel analysis of surface waves: Geophy sics, v. 64, n³, 1999, p. 800-808.

LCPC - Paramètres de forage en géotechnique. Méthode d'essai ME79, 2010, 54 p.

Norme NF P94-113 - Sols : reconnaissance et essais - Essai de pénétration statique. Soil: Investigation and testing - Cone penetration test, $1996,16 \mathrm{p}$.

Norme NF P11-300 - Classification des matériaux utilisables dans la construc tion des remblais et des couches de forme d'infrastructures routières, 1992 $21 \mathrm{p}$.

Norme NF P94-074 - Sols : reconnaissance et essais - Essai à l'appareil triaxial de révolution, 1994.

Norme NF P94-110-1 - Sols : reconnaissance et essais - Essai pressiométrique Ménard. Partie 1 : essai sans cycle. (Soil: Investigation and testing - Ménard pressure meter test - Part 1: Test without unload-reload cycle), 2000, $45 \mathrm{p}$.

XP P94-110-2 - Sols : reconnaissance et essais - Essai pressiométrique Ménard - Partie 2 : essai avec cycle. (Soil: Investigation and testing - Ménard pressuremeter test - Part 2: Test with unload Reload cycle) 1999, 8 p.

NF P94-157-1 - Sols : reconnaissance et essais - Mesures piézométriques Partie 1 : tube ouvert. (Soils: Investigation and testing. In situ pore pressure measurement. Part 1: Piezometric tube), $1996,12 \mathrm{p}$

NF P94-157-2 - Sols : reconnaissance et essais - Mesures piézométriques Partie 2 : sonde de mesure de pression interstitielle. (Soils: Investigation and testing. In situ pore pressure measurement. Part 2: Pore pressure meter) 1996 $16 \mathrm{p}$.

NF EN ISO 22475-1 - Reconnaissance et essais géotechniques - Méthodes de prélèvement et mesurages piézométriques - Partie 1 : principes techniques des travaux. (Geotechnical investigation and testing - Sampling methods and groundwater measurements - Part 1: Technical principles for execution), 2007, $135 \mathrm{p}$.

XP P94-202 - Sols : reconnaissance et essais - Prélèvement des sols et des roches - Méthodologie et procédures. Soil: investigation and testing. Soil sampling. (Methodology and procedures), 1995, 44 p.

NF EN ISO 22476-3 - Reconnaissance et essais géotechniques - Essais en place Partie 3 : essai de pénétration au carottier. (Geotechnical investigation and testing - Field testing - Part 3: Standard penetration test), 2005, $18 \mathrm{p}$.
Techniques Louis Ménard - Règles d'utilisation des techniques pressiométriques et d'exploitation des résultats obtenus pour le calcul des fondations. Brochure D60, 1965.

Norme NF EN 1997-1 - Eurocode 7, calcul géotechnique, partie I : règles générales. (Eurocode 7 : geotechnical design Part 1: General rules), 2005, $145 \mathrm{p}$

Norme NF EN 1997-1/NA - Eurocode 7 Calcul géotechnique - Partie 1 : règles générales - Annexe nationale à la NF EN 1997-1:2005. (Eurocode 7 - Geotechnical design - Part 1: General rules - National annex to NF EN 1997-1:2005), 2006, 10 p.

Norme NF EN 1997-2 - Eurocode 7 : calcul géotechnique - Partie 2 : reconnaissance des terrains et essais. (Eurocode 7: Geotechnical design - Part 2: Ground investigation and testing), 2007, $174 \mathrm{p}$.

LCPC/SETRA - Réalisation des remblais et des couches de forme. Guide technique Fascicule I Principes généraux ( $2^{\mathrm{e}}$ éd.) Réf. D9233-1, LCPC, ISBN 2110857072 , 2000, $98 \mathrm{p}$.

LCPC/SETRA 2000. Réalisation des remblais et des couches de forme. Guide technique. Fascicule II. Annexes techniques (2e éd.), Réf. D9233-2, LCPC ISBN 2110857080, $102 \mathrm{p}$

Document Technique Unifié DTU 13.11 Fondations superficielles (norme DTU P11-211). Contract bill for superficial foundations, contract bill of the specia clauses, 1988,14 p. + Modificatif 1 de 1997.

Document Technique Unifié DTU 13.12 Règles pour le calcul des fondations superficielles (norme DTU P11-711). Rules for the calculation of superficial foundations, $1988,18 \mathrm{p}$

Norme P11-212 - DTU 13.2 - Travaux de bâtiment - Travaux de fondations profondes pour le bâtiment - Partie 2 Cahier des clauses spéciales (Référence commerciale des normes P11-212 et NF P11-212-2), 1994, 86 p.

Norme NFP 94-500 - Missions d'ingénierie géotechnique - Classification et spécifications, Geotechnical engineering missions - Classification and specifications, 2006, $38 \mathrm{p}$

MELT (Ministère de l'Équipement, du Logement et des Transports) - Cahier des clauses techniques générales applicables aux marchés de travaux, Fascicule $n^{\circ} 62$, titre $V$ " Règles techniques de calcul et de conception des fondations des ouvrages de génie civil ». Textes officiels, $n^{\circ} 93-3,1993,182$ p. Cet ouvrage a également été édité par Eyrolles en 1999.

Norme NF P03-100 - Critères généraux pour la contribution du contrôle technique à la prévention des aléas techniques dans le domaine de la construction. General criteria for the contributio nof the technical inspection towards the prevention of technical risks in the construction field, 1995, $20 \mathrm{p}$.

Durand F. - CFMS, oct. 2009

\section{Éoliennes}

DIBT - Richtlinie für Windenergieanlagen. Deutches Institut für Bautecknik, Berlin. Directive allemande sur le dimensionnement des éoliennes, 2004, 45 p.
ISO 4354:1997 - Actions du vent sur les structures. Wind actions on structures, 1997, $52 \mathrm{p}$

Norme NF EN 61400-1 - Éoliennes-partie 1 : exigences de conception. (Wind turbinesPart 1), 2006, $93 \mathrm{p}$.

Norme NF EN 1991-1-4/NA - Eurocode 1 : Actions sur les structures - Partie 1-4: actions générales - Actions du vent Annexe nationale à la NF EN 1991-14:2005 - Actions générales - Actions du vent. (Eurocode 1: Actions on structures - Part 1-4: General actions - Wind actions - National annex to NF EN 1991-1-4:2005 - General actions - Wind actions), $2008,42 \mathrm{p}$

NF EN 1991-1-4 - Eurocode 1 : actions sur les structures -- Partie 1-4 : actions générales - Actions du vent. (Eurocode 1: Actions on structures - Part 1-4: general actions - Wind actions), 2005, $124 \mathrm{p}$.

Norme CEI 61400-2 - Aérogénérateurs Partie 2 : exigences en matière de conception des petits aérogénérateurs. (Wind turbines - Part 2: Design requirements for small wind turbines), 2006, $188 \mathrm{p}$

NF EN 60034-14 - Machines électriques tournantes - Partie 14: vibrations mécaniques de certaines machines de hauteur d'axe supérieure ou égale à $56 \mathrm{~mm}$ - Mesurage, évaluation et limites de l'intensité vibratoire. (Rotating electrical machines - Part 14: Mechanical vibration of certain machines with shaft heights $56 \mathrm{~mm}$ and higher - Measurement, evaluation and limits of vibration severity), 2004, $17 \mathrm{p}$

NF EN 60034-14/A1 - Machines électriques tournantes - Partie 14 : vibrations mécaniques de certaines machines de hauteur d'axe supérieure ou égale à $56 \mathrm{~mm}$ - Mesurage, évaluation et limites de l'intensité vibratoire. (Rotating electrical machines - Part 14: Mechanical vibration of certain machines withshaft heights $56 \mathrm{~mm}$ and higher - Measurement, evaluation and limits of vibration severity), 2007, 5 p.

\section{Autres références normatives}

NF X02-006 - Normes fondamentales. Le système international d'unités. Description et règles d'emploi - Choix de multiples et de sous-multiples. (Fundamental standards. The international systems of units. Description and rules for use. Choice of multiples and sub-multiples), 1994, 28p.

ISO 2394: 1998 - Principes généraux de la fiabilité des constructions. (General principles on reliability for structures), $1998,79 \mathrm{p}$

ISO $14688-1$ et $14688-2$

ISO $14689-1$ et $14689-2$.

\section{Articles}

Berthelot P., Lamadon Th. - Vérification des fondations d'éoliennes. Document Bureau Veritas Powerpoint. Stage CC 100 F, 28 nov. 2007,50 p.

Berthelot P., Lamadon Th. - Eoliennes. Reconnaissance géotechnique et principes de fondations. Document Bureau Veritas, nov. 2007, $16 \mathrm{p}$ 
Berthelot P., Frossard A., Glandy M. - Rigid inclusions: Calculation parameters and reception criteria for the distribution layer. ISSMGE, Mexico, 2006.

Berthelot P., Durand F., Frossard A., Glandy M. - Dallages et modules de déformation des couches de sol ; application aux renforcements de sols par inclusions et analyse du comportement du matelas de répartition. $14^{\mathrm{e}}$ Congrès européen, Madrid, 2007.

Berthelot P., Durand F., Glandy M. Méthode analytique du comportement d'un matelas de répartition au-dessus d'inclusions rigides et supportant ou non une structure en béton. $15^{\circ}$ Congrès européen, Athènes, 2011.

Bustamante M., Grau Ph., Le Kouby A. Cas des éoliennes. Document powerpoint présenté à la journée CFMS du $7 / 4 / 06$ ( sollicitations cyclique et fatigue des matériaux ) , 2006, 46 p

Faber T. - Offshore Wind Energy in Europe Actual Situation and Future Developments. Proceedings of the Sixteenth International Offshore and Polar Engineering Conference, Lisbon, Portugal, July 1-6, 2007.

Frank R. - Fondations profondes. Techniques de l'Ingénieur. C248, 1996, $45 \mathrm{p}$

Frank R., Zhao S.R. - Estimation par les paramètres pressiométriques de l'enfoncement sous charge axiale des pieux forés dans les sols fins. Bull. Liaison Labo P. et Ch. $\mathrm{n}^{\circ} 119$, mai-juin 1982 , p. 17-24.

Frossard A., Glandy M. - Justification d'une fondation superficielle sur un sol renforcé d'inclusions. Annales ITBTP, février 2002.

Houlsby G.T., Kelly R.B., Huxtable J., Byrne B.W. - Field trials of suction caissons in clay for offshore wind turbine foundations. Géotechnique 55, $\mathrm{n}^{\circ} 4$ 2005, p. 287-296.

Sieffert J.G., Cevaer F.-Manuel des fonctions d'impédance. Fondations superficielles. Ouest Éditions, Presses académiques, $1992,174 \mathrm{p}$

\section{Textes législatifs}

Décret n 93-1164 du 11 octobre 1993 relatif à la composition du cahier des clauses techniques générales applicables aux marchés publics de travaux et approuvant ou modifiant divers fascicules.

Loi $n^{\circ} 2005-781$ du 13 juillet 2005 de programme fixant les orientations de la politique énergétique.

Décret 91-1147 relatif aux Demandes de Renseignements (DR) concernant les réseaux existants et la liste des concessionnaires.

\section{Contrôle technique}

Article L. 111-23 du Code de la construction et de l'habitation, Partie législative, Livre Ir : Dispositions générales. Titre Ier : Construction des bâtiments. Chapitre Ir : Règles générales. Section 7 . Contrôle technique. Modifié par ordonnance $n^{\circ} 2005-658$ du 8 juin 2005 - art. 4 JORF 9 juin 2005.

Article L. 111-24 à 26 du Code de la construction et de l'habitation.

Article R 111-38 du Code de la construction et de l'habitation, Partie réglemen- taire, Livre Ier : Dispositions générales. Titre Ier : Construction des bâtiments. Chapitre $\mathrm{I}^{\mathrm{er}}$ : Règles générales. Section 7 : Contrôle technique. Sous-section 2 : Contrôle technique obligatoire. Modifié par Décret $n^{\circ}$ 2007-1327 du 11 septembre 2007 - art. 2 JORF 12 septembre 2007 en vigueur le 1 er octobre 2008.

Articles R 11-27 à 37 et 111-39 à 42 du Code de la Construction et de l'Habitation.

Décret $n^{\circ}$ 2007-1727 du 7 décembre 2007 relatif à l'extension du contrôle technique obligatoire à certaines constructions exposées à un risque sismique et modifiant le code de la construction et de l'habitation.

Loi $n^{\circ} 7$ 8-12 du 4 janvier 1978 relative à la responsabilité et à l'assurance dans le domaine de la construction (loi Spinetta).

Loi n $2008-735$ du 28 juillet 2008.

Décret $n^{\circ} 78-1146$ du 7 décembre 1978 Agrément des contrôleurs techniques.

NF P03-100 - Critères généraux pour la contribution du contrôle technique à la prévention des aléas techniques dans le domaine de la construction. General criteria for the contributionof the technical inspection towards the prevention of technical risks in the construction field $1995,20 \mathrm{p}$.

Loi n 83-440 du 2 juin 1983 Donnant force de loi à la $1^{\text {re }}$ partie (législative) du code de la construction et de l'habitation et modifiant certaines dispositions de ce code. Art. 2 (V) (modification de la lo Spinetta).

\section{Séismes}

Norme NF P06-013 - Règles de construction parasismique - Règles PS applicables aux bâtiments, dites règles PS 92 Earthquake resistant construction rules. Earthquake resistant rules applicable to buildings, called PS $92,1995,218 \mathrm{p}$

\section{Divers}

Article 121-3 du Code pénal, Partie législative, Titre II : De la responsabilité pénale. Chapitre $I^{\text {er }}$ : Dispositions générales. Modifié par la loi n² 2000-647 du 10 juillet 2000, art. 1 JORF 11 juillet 2000

Article 1792-1 du Code civil, Livre III : Des différentes manières dont on acquiert la propriété. Titre VIII : Du contrat de louage, Chapitre III : Du louage d'ouvrage et d'industrie. Section 3 : Des devis et des marchés.

Article 2270 du Code civil (au sujet de la garantie décennale)

Décret $n^{\circ}$ 95-20 du 9 janvier 1995 pris pour l'application de l'article L. 111-11-1 du code de la construction et de l'habitation et relatif aux caractéristiques acoustiques de certains bâtiments autres que d'habitation et de leurs équipements.

Décret $n^{\circ}$ 2007-1327 du 11 septembre 2007 relatif à la sécurité et à l'accessibilité des établissements recevant du public et des immeubles de grande hauteur, modifiant le code de la construction et de l'habitation et portant diverses dispositions relatives au code de l'urbanisme.

Loi $n^{\circ} 85-704$ du 12 juillet 1985 relative à la maîtrise d'ouvrage publique et à ses rapports avec la maîtrise d'œuvre privée.
Loi n²003-699 du 30 juillet 2003 relative à la prévention des risques technologiques et naturels et à la réparation des dommages.

Décret $n^{\circ}$ 2004-554 du 9 juin 2004 relatif à la prévention du risque d'effondrement de cavités souterraines et de marnières et modifiant le décret $n^{\circ}$ 90-918 du 11 octobre 1990 relatif à l'exercice du droit à l'information sur les risques majeurs.

Article L. 241-1 et 2 - Code des assurances sur l'assurance de responsabilité obligatoire des travaux de bâtiment

Article L. 242-1 et 2 - Code des assurances sur l'assurance de dommages obliga toire des travaux de bâtiment

Article L. 243-1 à 8-Code des assurances sur l'assurance obligatoire des travaux de bâtiment.

Code de l'environnement - Partie législative Livre V : Prévention des pollutions, des risques et des nuisances. Titre $V$ : Dispositions particulières à certains ouvrages ou installations. Chapitre III : Éoliennes.

\section{Autres documents}

Philipponnat G. - Paramètres dynamiques du sol. Fondations et ouvrages en terre. Eyrolles, 1997, p. 379-382.

Baugrunddynamik - DGGT, Berlin, 2002.

Grundbau-Taschenbuch (Part 1, chapter 1.8, section 4.2 , picture 38 )

Cahiers des charges techniques particuliers des différents constructeurs.

Combarieu O. - Calcul d'une fondation mixte, semelles-pieux, sous charge verticale centrée - Note d'information technique LCPC, 1988.

Annales Bâtiment Travaux Publics « Soulèvement de fondation m, oct.-nov. 1998.

CFMS (Comité Français de Mécanique des Sols et de géotechnique) - Recommandations sur la conception, le calcul, l'exécution et le contrôle des colonnes ballastées sous bâtiments et ouvrages sensibles au tassement. Revue française de géotechnique $n^{\circ} 111,2011,16$.

USG - Recommandations sur les investigations géotechniques pour la construction. Le Moniteur $n^{\circ} 5325-16$ décembre 2005. XP P 94-010 (1996), Sols : reconnaissance et essais - Glossaire géotechnique Définitions - Notations - Symboles décembre, AFNOR.

NF X02-006 - Normes fondamentales. Le système international d'unités. Description et règles d'emploi - Choix de multiples et de sous-multiples, 1994.

AFNOR - Présentation des rapports. Recommandation aux auteurs, 1991 $4 \mathrm{p}$.

Forage (NFP 94-500) : réalisation d'une excavation linéaire avec un outil et un procédé spécifiques aux techniques de percement.

Sondage (NFP 94-500) : exploration locale et méthodique d'un terrain à partir d'une excavation, d'un trou de forage de la pénétration d'une sonde ou de l'utilisation de techniques physiques non ou peu destructives, pour en determiner la nature, la structure ou effectuer des mesures de propriétés physiques ou chimiques, mécaniques ou hydrauliques. 